\title{
Abstracts from HSG 2019
}

Published online: 8 October 2019

(C) The American Society for Experimental NeuroTherapeutics, Inc. 2019

Quality of Care for Huntington's Disease in the United States: Findings from a National Survey of Patients and Caregivers

Karen E. Anderson ${ }^{1}$, Jack Griffin ${ }^{2}, \mathrm{Al} \mathrm{Kinel}{ }^{3}$, Abdul R. Shaikh $^{4}$, Temitope Olofintuyi ${ }^{4}$, Stevan Ramirez ${ }^{5}$, Joni Steinman ${ }^{3,5}$, George J. Yohrling $^{6}$, Shari Kinel ${ }^{5}$

${ }^{1}$ Georgetown University, Department of Psychiatry \& Department of Neurology, Washington DC, USA; ${ }^{2}$ Griffin Foundation, Naples, FL, USA; ${ }^{3}$ Strategic Interests, LLC, Rochester, NY, USA; ${ }^{4}$ Guidehouse LLP, Washington DC, USA; ${ }^{5}$ Huntington Study Group, Rochester, NY, USA; ${ }^{6}$ Huntington's Disease Society of America, New York, NY, USA

Background: Little is known about the quality of care for people living with Huntington's disease (HD) in the United States.

Objective: To enhance our understanding of the HD care experience for patients and families, and identify gaps in care provision in the United States. This study focused specifically on care needs outside of routine clinic visits.

Methods: Web-based survey for persons self-identifying as being affected by HD (PAHD, which included individuals with, or at risk for HD) or as caregivers/family members were disseminated via social media and patient advocacy partners from April-May 2017. Survey completion required 5$10 \mathrm{~min}$

Results: Total responses were 797, $(n=585$ caregiver/family respondents; $n=212$ PAHD) from 45 states. Overall 459 (57.6\%) answered questions about current level of care provision. Of these, $41.0 \%$ reported receiving medical care, $5.7 \%$ community care, $31.4 \%$ both medical and community care, and $22.0 \%$ reported having no current medical or community care. Top reasons cited for receiving 'No other care support for HD' $(n=101)$ were "high cost of care" (27.7\%) and "live too far away" (26.7\%). Caregiver/ family respondents reported helping the affected individual with budget/ finances $(60.5 \%)$, housekeeping $(57.1 \%)$, daily help $(53.2 \%)$, finding community resources $(42.3 \%)$, safety $(38.1 \%)$, medical care $(33.9 \%)$, home physical therapy $(16.0 \%)$, and meals on wheels $(5.9 \%)$. Questioning about healthcare visits revealed that patients were only given information/ discussed next steps half $(53.1 \%)$ of the time, but questions about depression and other mental health issues were more routinely explored (89.9\%).

Conclusions: A significant proportion of HD care is currently provided by caregivers at home, greatly magnifying the burden of disease. Enhancing resources for both formal and informal care for patients and families should be a central goal for HD organizations. Development of patient/caregiver outcome measures to track impact of any interventions is crucial.

Lay Summary: This project identifies gaps in care that are experienced by people with Huntington's Disease and their families. Areas where there are not enough support or resources include care given outside of the clinic, such as in home care, and training non-specialists in how to care for people with Huntington's Disease. The next phase of this project will seek solutions to reduce the burden of care for all people affected by Huntington's Disease.
2

Behavioral Distinctions Between Physical and Cognitive Apathy Observed in Premanifest Huntington's Disease (HD)

Kelly Atkins, Sophie Andrews, Julie Stout, Trevor T-J Chong Turner Institute for Brain and Mental Health, Monash University, Melbourne, Victoria, Australia (all authors)

Patient reported outcomes clearly document apathy as a pervasive problem in HD. We sought to examine motivational deficits with an economic decision-making task, thus providing a performance based outcome measure of apathy. Motivation may be defined as a willingness to exert effort for rewards. Not all effort is the same; physical demands may be valued differently to cognitive ones. Little is known about how these effort domains are affected in premanifest HD.

We administered an economic effort-based decision-making task to 20 people confirmed to have the HD gene and 20 matched controls. Participants were first trained extensively on two tasks requiring effort that was either primarily cognitive or physical. They then had to select between a baseline low level of effort for a low reward option, and a high effort for a higher reward option. Separate choices were made for the cognitive and physical tasks, which facilitated computational models of motivation in each effort domain. Our premanifest HD participants $\left(\mathrm{DBS}_{(\text {range) }} 150-435\right)$ demonstrated similar levels of physical motivation to controls in that both groups were equally likely to invest physical effort for reward. In contrast, our presymptomatic HD group consistently preferred the less cognitively effortful option, $F(1,45)=7.56, p<.05$, suggesting that they were less motivated than controls to invest cognitive effort for reward.

These data suggest that physical and cognitive motivation are dissociable in pre-symptomatic HD and that cognitive apathy may occur earlier in the disease course than physical apathy. These findings contribute to our current understanding of the dissociations between physical and cognitive costs, which are central to current conceptualisations of apathy in HD. Moreover, our results support the clinical utility in defining distinct subtypes of apathy, and the relevance of these subtypes when designing interventions or clinical trials targeting apathetic states.

Lay Summary: Our study describes a novel and objective way of measuring apathy, a pervasive psychiatric symptom of HD, that may be useful for trials or interventions targeting apathy. Moreover, our results suggest people with the HD gene may experience a type of cognitive apathy, prior to the development of physical symptoms with which a diagnosis of HD is made.

3

Multidimensional Apathy in Huntington's Disease: The Utility of the Dimensional Apathy Scale 
Kelly Atkins, Sophie Andrews, Trevor T-J Chong, Julie Stout Turner Institute for Brain and Mental Health, Monash University, Melbourne, Australia (all authors)

Recent conceptualisations of apathy describe distinct subtypes (executive, initiation, and emotional) that differentially affect goal-directed behaviour, however, the presence of these subtypes over the disease course has not been described in HD. The aim of this study was to evaluate the use of a selfreport measure of these subtypes, the Dimensional Apathy Scale (DAS), in people with pre-manifest and manifest $\mathrm{HD}$ and their care partners.

72 people with the HD gene ( 38 pre-symptomatic and 34 manifest) alongside 72 matched controls completed the DAS and the Total Functioning Capacity (TFC). A subset of 38 partners completed the informant versions of all measures.

Higher TFC scores were associated with higher scores on the executive, $r=-.47^{*}$, initiation, $r=-.53 *$ and emotional, $r=-.51^{*},\left(p<.05^{*}\right)$ apathy subtypes. Relative to healthy controls and the pre-symptomatic group, the manifest HD group endorsed higher levels of apathy on executive, $F=$ $16.96, p=<.001$, and initiation subscales $F=14.61, p=<.001$, with large effect sizes, and on the emotional subscale $F=7.84, p=.001$, with a moderate effect size. Across the HD group, internal consistency reliability was found to be good for executive $(\alpha=0.82)$ and initiation $(\alpha=0.86)$ subscales, but very poor for the emotional subscale $(\alpha=0.27)$. Likewise, interrater reliability between the HD group and their respective informant was adequate for executive and initiation subscales (ICC > .7), but poor for the emotional subscale (ICC $=.45$ )

Manifest HD participants endorsed higher apathy levels than premanifest or control participants on all three subtypes of apathy on the DAS, suggesting that the apathy observed in HD has pervasive impacts across various forms of goal-directed behaviour. The association between DAS subscale scores and TFC suggests that the DAS is sensitive to disease progression, at least after the onset of functional decline. Low internal and interrater reliabilities on the emotional subscale, however, indicate that this subscale should be interpreted with caution.

Lay Summary: Our review of a commonly used patient reported apathy measure will assist clinicians to better recognize and assess apathy, a pervasive psychiatric symptom, in Huntington's disease.

Presymptomatic Testing and Confidentiality in the Age of the Electronic Medical Record

Stacey K. Barton, Kevin J. Black, Joel S. Perlmutter

Washington University School of Medicine, St. Louis, MO, USA (all authors)

Background: For over 20 years, presymptomatic testing for Huntington disease (HD) has been conducted using accepted guidelines meant to support and educate patients seeking testing while maximizing confidentiality and minimizing the risk of genetic discrimination. Advances in electronic medical records (EMRs) have created challenges in meeting these guidelines that include in-person disclosure of test results vs. patient portal access and risk of unintended disclosure via the EMR to those outside of the testing team.

Methods: We reached out to 13 HDSA Centers of Excellence with questions relevant to our concerns.

Results: Ten institutions responded; several requested not to be identified, often regarding concern about their own practices. All documented presymptomatic testing encounters in the EMR, although two used a pseudonym and one system was not linked to the primary EMR. Just over half allowed the use of a pseudonym, however, at least one institution did not sanction the practice. Many institutions intentionally documented little information in the EMR note to obscure the visit diagnoses. Over half had different processes for self-pay patients such as using an outside lab rather than the hospital lab. Most could avoid documenting the test report in the EMR, and one site did not document the visit that revealed test results. All could prevent test results provided through the patient portal before in-person disclosure. Several institutions had agreements with their laboratory directors to order the HD test results using a generic name such as "genetic analysis" and/or to provide results only on paper.

Discussion: There is no consensus among nationally respected institutions as to how to resolve the conflicts between the advantages of a shared medical record and the ability to maintain control of highly confidential medical information. More importantly, current practices potentially compromise patient confidentiality.

Lay Summary: It is important for patients to understand institutional practices of documenting HD testing in the electronic medical record (EMR). There is no consensus among peer institutions and many practices leave patients open to potential genetic discrimination and unable to control access to their results in their EMR.

5

\section{Influence of Age of Onset on Huntington's Disease Phenotype}

Lauren Kwa, Danielle Larson, Chen Yeh, Danny Bega

Northwestern University Feinberg School of Medicine, Chicago, IL, USA (all authors)

Background: Huntington's Disease (HD) is an inherited neurodegenerative disorder that is characterized by progressive motor, cognitive, and behavioral deficits [1]. Average age of onset is 40 years old [2,3]. It has been suggested that older onset patients may have a milder disease course with decreased odds of behavioral symptoms at onset [4]. However, phenotypic differences based on age of onset have not been well characterized in a large HD population.

Objectives: To use a large database to determine the difference in symptom profiles and disease progression between patients with early onset, typical onset, and late onset adult HD.

Methods: Data were obtained from Enroll-HD. Adults with manifest/ motor-manifest HD were included. Age groups were defined as follows: young onset (20-29 years), typical onset (30-50 years), and late onset (51+ years). Subjects in each group were categorized by TFC total score bins, from Stage I (least severe) to Stage V (most severe). Motor, cognitive, and behavioral symptoms were analyzed annually. Descriptive statistics were calculated. Chi-square test and Kruskal-Wallis test, as appropriate, were used to determine $p$ values and Bonferroni $p$ value correction was used to do pairwise comparison.

Results: 7311 manifest HD subjects were analyzed of which 612 were young onset, 4189 typical onset, and 2510 late onset. The rate of decline in TFC score from baseline to visit 1 was significantly faster in the young onset participants $(-1.79$ points $)$ compared to the typical $(-1.21$ points, $p<0.05)$ or late onset $(-1.15$ points, $\mathrm{p}<0.05)$ participants. Additional analyses of differences between age-ofonset groups while controlling for TFC score are presented for motor, cognitive, and behavioral variables.

Conclusions: Young age of onset is predictive of a faster functional decline for adults with HD when compared to those with typical and late age of onset. Motor and behavioral symptom profiles differ based on age of onset.

\section{References:}

1. Suchowersky, Oksana. "Huntington disease: Clinical features and diagnosis." UpToDate, www.uptodate.com/contents/huntingtondisease-clinical-features-and-diagnosis.

2. Myers, Richard H. "Huntington's disease genetics." NeuroRx: The Journal of the American Society for Experimental 
NeuroTherapeutics vol. 1,2 (2004): 255-62. doi:10.1602/ neurorx.1.2.255

3. Duyao, M., et al. "Trinucleotide repeat length instability and age of onset in Huntington's disease.” Nature Genetics 4.4 (1993): 387.

4. Ranganathan, Megha. "The Relationship Between Age-of-Onset and the Behavioral Phenotypic Manifestations in Huntington's Disease." Electronic Thesis or Dissertation. Ohio State University, 2018. OhioLINK Electronic Theses and Dissertations Center. 11 Dec 2018.

Lay Summary: Our study has the potential to aid in prognostication and inform the standard of care and recommended therapies for patients with Huntington's disease depending on their age of onset.

Evaluating Verbal Memory Function in Premanifest Huntington's Disease

Ilex Beltran-Najera ${ }^{1}$, Lisa V. Graves ${ }^{2}$, Heather M. Holden ${ }^{2}$, Savanna M. Tierney $^{3}$, Jordan Castleton ${ }^{4}$, Dean C. Delis ${ }^{5}$, Jody Corey-Bloom ${ }^{4}$, Paul E. Gilbert $^{1,2}$

${ }^{1}$ Department of Psychology, San Diego State University, San Diego, CA; ${ }^{2}$ San Diego State University-University of California San Diego Joint Doctoral Program in Clinical Psychology, San Diego, CA; ${ }^{3}$ Department of Psychology, University of Houston, Houston, TX; ${ }^{4}$ Department of Neurosciences, University of California San Diego, La Jolla, CA; ${ }^{5}$ Department of Psychiatry, University of California San Diego, La Jolla, CA

Objective: The purpose of the current study was to evaluate memory function in premanifest gene carriers for Huntington's disease (Pre-HD) by comparing performance on the California Verbal Learning Test-II (CVLT-II) in Pre-HD with that of healthy adult (HA) and manifest HD subjects.

Background: Verbal memory impairment is well documented in $\mathrm{HD}$; however, less is known about the nature and extent of memory deficits in Pre-HD. The CVLT-II is a standardized well-validated test of verbal learning and memory that assesses immediate free recall, free and cued recall over short and long delays, and yes/no recognition memory.

Methods: Participants included 77 HD, 46 Pre-HD, and 54 HA subjects followed at the UCSD Huntington's Disease Center of Excellence. Recall, recognition, learning characteristics, errors, and retention were analyzed using the CVLT-II, which was administered using standard procedures (Delis et al., 2000) and scored using CVLT-II software (Delis \& Fridlund, 2000).

Results: Pre-HD participants performed significantly worse than HA (ps $<.05$ ), but significantly better than HD (ps <.001) participants on Total Immediate Recall, Short and Long Delay Recall (Free and Cued), and Recognition Discriminability. Pre-HD individuals did not differ from the HA group, but performed significantly better than the HD group, on Learning Slope Trials 1-5, Semantic Clustering, and Intrusions (ps $<.001)$. Finally, there were no group differences in Repetitions or Retention.

Conclusions: We conclude that subtle declines in immediate and delayed recall may be evident prior to clinical diagnosis of HD, while more frontally-mediated processes, such as strategic aspects of encoding (i.e. semantic clustering), some error types (e.g. intrusions), and retrieval problems (demonstrated by better recognition versus recall) may be less impacted in the earliest stages of disease progression.

Lay Summary: The objective of this study was to evaluate memory function in premanifest gene carriers for HD. We found that subtle declines in immediate and delayed recall may be evident even prior to clinical diagnosis of HD.
The Effects of Balanced-Based Torso Weighting (BBTW) on Mobility in People with Huntington's Disease: A Case Series Experience

Christina Burke $^{1}$, Stacy Trebing ${ }^{2}$, Nicholas Froiseth ${ }^{2}$, Mark Gudesblatt ${ }^{2}$ ${ }^{1}$ Stony Brook University, Stony Brook, NY, USA; ${ }^{2}$ South Shore Neurologic, Patchogue, NY, USA

Background: Huntington's Disease (HD), a progressive neurodegenerative disorder, impacts cognition, behavior and gait resulting in mobility limitations and increased fall-risk. BalancedBased Torso Weighting (BBTW), an orthotic device, improves postural control by strategically placed weights on the individual's torso. BBTW use has improved gait and balance in select people with multiple sclerosis and cerebellar ataxia with mobility limitations.

Purpose: To evaluate the potential effect of BBTW in people with HD (PwHD).

Case Description: Two PwHD who experienced chorea, mobility deficits and reported a fall history were evaluated. Subject 1: $25 \mathrm{y} / \mathrm{o}$ PwHD self-reported fall history 2-3x/day. Subject 2: 62 y/o PwHD self-reported fall history as several times/month.

Design/Method: Both PwHD were assessed with BalanceWear vest utilizing standardized BBTW assessment protocol. Balance and gait outcome measures were performed both pre and immediately post vest application. Outcome measures included: Sensory Organization Test (SOT, (Smart Balance Master)), Gait Velocity and Step Length (GV, SL, ZenoMat), Functional Gait Assessment (FGA), 5x Sit To Stand Test (5XSTS).

Outcomes:

Subject 1: SOT demonstrated 19\% improvement (52 pre-test, 62 posttest). GV with cognitive dual task (serial 3's) increased $11.9 \%(101 \mathrm{~cm} / \mathrm{s}$ pre-test, $113 \mathrm{~cm} / \mathrm{s}$ post-test). FGA improved $23.9 \%$ (20/30 pre-test, $25 / 30$ post-test).

Subject 2: 5 XSTS improved 20\%, (18 s pre-test, 15 s post-test). GV increased $15 \%(60 \mathrm{~cm} / \mathrm{s}$ pre-test, $69 \mathrm{~cm} / \mathrm{s}$ post-test $)$, with left SL increasing $29 \%$ and right SL increasing $17 \%$ (34 cm pre-test, $44 \mathrm{~cm}$ post-test; $45 \mathrm{~cm}$ pre-test, $53 \mathrm{~cm}$ post-test respectively). FGA scores improved $78 \%$ (11/30 pre-test, $20 / 30$ post-test).

\section{Discussion:}

BBTW application demonstrated immediate impact in improving balance and ambulation in two PwHD as documented by multiple standardized outcome measures of balance and gait. BBTW may have potential for improving PwHD postural control reduce fall-risk. Further research is needed to confirm these preliminary findings, identify optimal candidates, and determine the long term impact of BBTW in reducing PwHD fall-risk. Lay Summary: This case study demonstrated the positive effect of placing a weighted vest on two individuals with HD. There was an immediate improvement in both balance and mobility. For certain individuals, wearing this vest can improve balance and reduce falls in people with HD.

8

Longitudinal Evaluation of Mutant Huntingtin and Neurofilament Light as Biomarkers for Huntington's Disease: The HD-CSF Study 24-month Analysis

Lauren M Byrne ${ }^{1}$, Filipe B Rodrigues ${ }^{1}$, Rosanna Tortelli ${ }^{1}$, Eileanoir B Johnson $^{1}$, Peter A Wijeratne ${ }^{2}$, Marzena Wylezinska-Arridge ${ }^{3}$, Enrico De Vita $^{3,4}$, Daniel C Alexander ${ }^{2,5}$, Scott Schobel ${ }^{6}$, Rachael I Scahill ${ }^{1}$, Amanda Heslegrave ${ }^{7}$, Henrik Zetterberg ${ }^{7,8,9}$, Edward J Wild ${ }^{1}$

${ }^{1}$ Huntington's Disease Centre, UCL Queen Square Institute of Neurology, London, UK; ${ }^{2}$ Centre for Medical Image Computing, Department of Computer Science, UCL, London, UK; ${ }^{3}$ Lysholm Department of Neuroradiology, National Hospital for Neurology and 
Neurosurgery, London, UK; ${ }^{4}$ Department of Biomedical Engineering, School of Biomedical Engineering and Imaging Sciences, King's College London, UK; ${ }^{5}$ Clinical Imaging Research Centre, National University of Singapore, Singapore; ${ }^{6}$ Neuroscience, Ophthalmology, and Rare Diseases (NORD), Roche Pharma Research and Early Development, Roche Innovation Center Basel, F. Hoffman-La Roche Ltd., Basel, Switzerland; ${ }^{7}$ UK Dementia Research Institute at UCL, London, UK; ${ }^{8}$ Department of Psychiatry and Neurochemistry, Institute of Neuroscience and Physiology, the Sahlgrenska Academy at the University of Gothenburg, Mölndal, Sweden; ${ }^{9}$ Clinical Neurochemistry Laboratory, Sahlgrenska University Hospital, Mölndal, Sweden

Background: There is a pressing need for sensitive biomarkers in Huntington's disease (HD). Mutant huntingtin (mHTT) and neurofilament light (NfL) have been proposed as potential biofluid biomarkers for application in clinical trials. However, little is known about their longitudinal dynamics during the natural history of HD.

Aims: We aimed to characterize the longitudinal trajectories of mHTT in CSF and NfL in CSF and blood plasma.

Methods: We used 24-month longitudinal data from the HD-CSF study. CSF mHTT, CSF NfL and plasma NfL were measured using immunoassays. Participants underwent standardized clinical assessments, CSF and blood collections, and optional brain MRI. Data were modeled using mixed effects models, and associations with clinical and imaging outcomes was studied with Pearson's partial correlations. Receiver operating characteristics curves were used to test discriminative ability, and samples size calculations to assess feasibility for clinical trials.

Results: $93 \%$ of the participants completed the 24-month follow-up. CSF mHTT, CSF NfL and plasma NfL increased over time in HD mutation carriers and had trajectories distinct from healthy controls. A single measurement at baseline of each analyte significantly predicted subsequent brain atrophy and change in clinical outcomes, including the composite UHDRS. Rate of change in the analytes showed weak associations with change in clinical or imaging outcomes. Baseline measurements for each analyte had better accuracy than rate of change in analyte for distinguishing between controls and HD mutation carriers, and between premanifest and manifest HD. Sample size calculations provide participant numbers needed to incorporate these biomarkers into clinical trials. Conclusion: This longitudinal study provides the first characterization of how mHTT and NfL fluctuate with time in HD mutation carriers. These data will facilitate the interpretation of drug-related alterations in mHTT and NfL in current and future clinical trials.

Lay Summary: Having sensitive biomarkers that respond when a drug is working could help make clinical trials run faster and with smaller numbers of people. In order to understand drug related changes in biomarkers we need to know how they alter in HD mutation carriers without a drug. This study will help us do this.

\section{Enroll-HD Study Status}

The Enroll-HD Platform Team

CHDI Foundation, USA (all authors)

Background: Enroll-HD is a clinical research platform that includes at its core an observational, prospective study of HD. The objectives of EnrollHD are: 1) enhance the design and expedite the conduct of clinical trials; 2) improve the understanding of the phenotypic spectrum and disease mechanisms; 3) foster good clinical care.

Over 21,500 participants $(18,734$ currently active) have been recruited from 19 countries and 163 study sites. The data collected from these participants is monitored using a rigorous risk-based process. Recoded data and biosamples are made available to researchers.

Enroll-HD also serves as a registry that can be used to facilitate recruitment, by identifying potentially eligible participants who can be invited by investigators to participate in clinical trials. Selection criteria (e.g. local CAG, UHDRS TMS) can be queried to generate listings of participants potentially eligible for a given clinical trial. To improve support for future clinical trials and observational studies, the study has been focusing on the recruitment of at risk, premanifest and early-stage participants. The implementation of the new recruitment strategy is positively impacting the participant population, with an increase of the desired cohorts.

Platform studies are clinical studies that utilize at least one or more Enroll-HD platform services. These services include site feasibility, study guidance documents and templates, potentially eligible participant listings, study set-up support, monitoring and data management. The Enroll-HD Clinical Training Portal is an online resource launched in January 2017 where HD research personnel can complete and maintain study-relevant training, presently UHDRS Motor Certification, GCP (all users), and Enroll-HD Plasma Collection (Enroll-HD study users only). There are currently more than 1600 active users, and it enables faster, more cost-effective start-up of clinical trials and studies, standardizes the quality of training, and reduces workload at sites.

Lay Summary: Enroll-HD is a clinical research platform that includes an observational study of Huntington Disease (HD). Eligible participants include individuals who are gene expansion carriers, individuals with unknown genotype and controls. Data are collected annually on motor, cognitive and behavioral symptoms, releasing coded data to researchers periodically. Enroll-HD improves support for clinical trials and provides high-quality clinical data and biological samples. As a research platform, Enroll-HD brings together a novel set of tools for HD clinical research that is easily available for researchers.

\section{0}

Network Based Approaches to Investigate the Relationship of Metabolites Related to Huntington's Disease with Phenols in Olive Oil

Christiana C. Christodoulou ${ }^{1,2,3}$, Christiana A. Demetriou ${ }^{3,4}$, Yiolanda Christou $^{2,3}$, George M. Spyrou ${ }^{1,3}$, Eleni Zamba-Papanicolaou, ${ }^{2,3}$

${ }^{1}$ Bioinformatics Group, CING; ${ }^{2}$ Neurology Clinic D, CING; ${ }^{3}$ Cyprus School of Molecular Medical School; ${ }^{4}$ Department of Primary Care and Population Health, University of Nicosia Medical School

Introduction: Huntington's Disease (HD) is an inherited autosomal dominant neurodegenerative disease affecting the medium spiny neurons of the basal ganglia [1]. Exposure to the Mediterranean Diet (MD), which is high in anti-oxidants, provides neuroprotection, assisting the body's innate anti-oxidant system to eliminate ROS species [2], [3], [4].

Metabolites are small compounds and the intermediate end-products of metabolism. Metabolomics is the systematic study of metabolites from a biological sample. Metabolomics are proving to be useful in the study of diseases as they are not only closer to the phenotype but may provide possible biomarkers for disease.

The Human Metabolome Database (HMDB) [5] is a freely available database containing information on metabolites found in the human body. HMDB will be used to retrieve metabolites from a list of HD genes based on our previous work.

Aim of study:

i) Metabolomic profile of human serum samples using untargeted MSbased approaches for identification of metabolites and their associated pathways that significantly differentiate pre-symptomatic from early manifest and advanced manifest patients with HD and therefore might be involved in phenoconversion and disease severity.

ii) Metabolomic profiles of human serum samples using untargeted MSbased approaches, to identify metabolites and their associated mechanisms influenced by olive oil consumption and the MD. 
iii) Examine whether the mechanisms perturbed in different disease stages are also influenced by olive oil consumption and the MD, providing molecular support to the hypothesis that olive oil consumption and MD adherence can positively affect HD onset and severity.

Materials and methods: This is a case-control study consisting of presymptomatic $(n=20)$, early symptomatic $(n=20)$ and advanced stage $(n=16)$ HD patients as well as age matched controls $(n=56)$. Data regarding demographics, clinical characteristics and lifestyle were collected, including information on dietary intakes using a detailed Food Frequency Questionnaire (FFQ).

Untargeted metabolomics analysis will be performed using Liquid chromatography-Mass spectrometry (LC-MS) approaches on serum samples obtained from Cypriot HD patients and healthy controls. Interpretation of metabolomics data will be performed using different bioinformatics tools and databases to identify metabolites and their associated mechanisms influenced by olive oil consumption and the MD.

Results: Expected to demonstrate an overlap between the pathways/ metabolites perturbed in HD and those influenced by olive oil intake and MD adherence, thus providing biological plausibility to the available epidemiological evidence, further supporting the neuroprotective effects of olive oil and the MD. Therefore, highlighting the potential use of the MD and olive oil in non-pharmacological interventions aiming to delay HD onset and progression.

Funding Information: European Commission Research Executive Agency (REA) Grant BIORISE (Num. 669,026), under the Spreading Excellence, Widening Participation Science with and for Society Framework. World Olive Health Centre and the A.G. Leventis Foundation.

\section{References:}

1. Johri, A. and Beal, M. (2012). Antioxidants in Huntington's disease. Biochimica et Biophysica Acta (BBA) - Molecular Basis of Disease, 1822(5), pp.664-674.

2. Sofi F, Macchi C, Casini A. Mediterranean Diet and Minimizing Neurodegeneration. Curr Nutr Rep 2013; 2: 75-80.

3. Valls-Pedret C, Sala-Vila A, Serra-Mir M, et al. Mediterranean Diet and Age-Related Cognitive Decline: A Randomized Clinical Trial. JAMA Intern Med 2015; 175: 1094-103.

4. Selmin OI, Romagnolo APG, Romagnolo DF. Mediterranean Diet and Neurodegenerative Diseases. In: Romagnolo DF, Selmin OI, eds. Mediterranean Diet. Springer International Publishing, 2016: $153-64$.

5. http://www.hmdb.ca

Lay Summary: Identify pathways/metabolites in HD and those influenced by olive oil intake and MD adherence in Cypriot HD patients using untargeted metabolomics analysts and bioinformatics analysis, tool and databases. I believe this is important to the HD community as it provides biological plausibility to the available epidemiological evidence, further supporting the neuroprotective effects of olive oil and the MD and also the potential use of MD and olive oil in non-pharmacological interventions aiming to delay HD onset and progression. The use of metabolomics may provide early biomarkers for HD detection.

11

Neurocognitive Function in Adolescent and Young Adult Offspring of Huntington's Disease Patients

Abagail E. Ciriegio ${ }^{1}$, Lisa $\mathrm{Hale}^{2}$, Anna C. Pfalzer ${ }^{2}$, Katherine McDonel1 ${ }^{2}$, Heather Riordan ${ }^{2}$, Sarah Moroz ${ }^{2}$, Daniel O. Claassen $^{2}$, Bruce E. Compas ${ }^{1}$
${ }^{1}$ Vanderbilt University, Nashville, TN, USA; ${ }^{2}$ The Huntington Disease Society of America Level 1 Center of Excellence at Vanderbilt University Medical Center, Nashville, TN, USA

It is well documented that patients with Huntington's disease (HD) experience pronounced problems in cognitive function including domains of complex executive function. Due to the fact that HD is typically viewed as an adult onset neurodegenerative condition, research has focused on charting the course of cognitive function in adults (e.g., PREDICT-HD). However, relatively little research has examined the possible early emergence of cognitive problems in individuals at risk for HD. As part of a new program of research to re-conceptualize HD as a neurodevelopmental disorder, we have examined multiple dimensions of cognitive function in adolescent (ages 10-19 years; $n=16$ ) and young adult (ages 20-29 years; $n=8$ ) offspring of HD patients (age 30 and above, $n=13$ ). We assessed working memory using the Working Memory Index (WMI) from the WISC-V and WAIS-IV. Further, we examined multiple dimensions of executive function using the NIH Toolbox (NIHTB) Cognitive Battery (e.g., inhibition and attention, working memory, episodic memory). Our initial findings are striking as all of these cognitive measures were significantly and negatively correlated with age. For example, the WMI from the WISC-V and WAIS-IV was negatively correlated with age $(r=-.53, p=.001)$. Consistent with findings of previous studies with adult HD patients, we found evidence for a progressive decline in attention and inhibition from age 10 through adulthood from the Flanker Inhibitory Control and Attention Task $(r=-.40, p=.013)$. In addition, over half of the adolescents and young adults scored below the normative mean on this scale and 3 adolescents and 3 young adults had standard scores below 80 suggesting significant impairment in attention and inhibition even at these early ages. Our findings underscore the importance of examining the developmental course of cognitive problems in HD patients and their offspring across the lifespan.

Lay Summary: As part of a new program of research to reconceptualize HD as a neurodevelopmental disorder, we have examined multiple dimensions of cognitive function in a sample of HD patients and their children. Our findings underscore the importance of examining the developmental course of cognitive problems in HD patients and their offspring across the lifespan.

\section{2}

Understanding the Effects of Stress in Families Impacted by Huntington's Disease: Associations of Coping and Psychiatric Symptoms

Abagail E. Ciriegio ${ }^{1}$, Lisa Hale ${ }^{2}$, Anna C. Pfalzer $^{2}$, Katherine McDonell ${ }^{2}$, Heather Riordan ${ }^{2}$, Sarah Moroz ${ }^{2}$, Daniel O. Claassen ${ }^{2}$, Bruce E. Compas ${ }^{1}$ ${ }^{1}$ Vanderbilt University, Nashville, TN, USA; ${ }^{2}$ The Huntington Disease Society of America Level 1 Center of Excellence at Vanderbilt University Medical Center, Nashville, TN, USA

Huntington's disease (HD) can be conceptualized as a disorder that affects both patients and families. The stressors for patients resulting from the progression of the disease are well documented. However, sources of stress and their impact on offspring of HD patients are less understood. These offspring are faced with stressors that are chronic, unpredictable, and uncontrollable. Further, it is essential to examine the ways adolescent and young adult offspring cope with the stress of being from a family impacted by HD. Previous research has shown that the use of coping strategies that include acceptance and cognitive reappraisal (i.e., secondary control coping) is most adaptive for unpredictable and uncontrollable stress. We obtained reports of coping with HD related stress and psychiatric symptoms from a sample of adolescent $(10-19$ years; $n=16)$ and young adult (20-29 years; $n=8$ ) offspring of HD patients. First, levels of a wide range of psychiatric symptoms including depression, anxiety, and 
obsessive-compulsive problems were elevated in these offspring. The use of secondary control coping in response to HD related stress was significantly and negatively correlated with symptoms of depression $(r=-.45$, $p=.036)$, anxiety $(r=-.57, p=.005)$, and obsessive-compulsive behaviors $(r=-.56, p=.006)$. To our knowledge, this is the first study to empirically document the association of coping and psychiatric symptoms in adolescent and young adult offspring of HD patients. Importantly, these findings suggest that teaching secondary control coping skills (e.g., acceptance, cognitive reappraisal) may be important in building resilience in these offspring of HD patients.

Lay Summary: Families impacted by Huntington's disease are faced with stressors that are chronic, unpredictable, and uncontrollable. To our knowledge, we are the first study to empirically document the association of coping with these stressors and psychiatric symptoms in adolescent and young adult offspring of HD patients.

\section{3}

\section{Gene Expression Profiling of Depression in Huntington's Disease}

Gabriela D. Colpo, Natalia P. Rocha, Erin Furr Stimming, Antonio L. Teixeira

McGovern Medical School, The University of Texas Health Science Center at Houston, Houston, TX, USA (all authors)

Background: Besides motor symptoms, Huntington's disease (HD) is marked by behavioral changes. Among psychiatric symptoms, depression is of great relevance as it is considered a significant burden. In addition, the presence of depression is a predictor of suicide. This preliminary study investigates the gene expression profile by RNA-seq in HD patients with and without depression, and between subjects with HD and healthy controls.

Methods: We performed a RNA-seq analysis of blood of HD patients with depression $(n=8)$, HD patients with no depression or history of depression $(n=8)$ and healthy control subjects $(n=8)$, all matched for age. Preprocessing of data and between-group comparisons were calculated using DESeq2. The Wald test was used to generate $p$ values and $\log 2$ fold changes. Genes with a p value $<0.05$ and absolute $\log 2$ fold change $>1$ were called as differentially expressed genes. In addition, we clustered differentially expressed genes by their gene ontology (GO) using GeneSCF and the enrichment of GO terms was tested by Fisher exact test.

Results: In this exploratory analysis, we found 19 genes differently expressed between HD patients with depression and HD without depression. Among these genes, 6 were upregulated and 13 downregulated. According to the GO analysis, these genes are involved in pathways such as apoptotic process and immune response. In the analysis between all HD patients $(n=16)$ and healthy controls we found 60 genes differently expressed, of which 21 were upregulated and 39 downregulated. According to the GO analysis, these genes are involved in neuron migration, neuron cell-cell adhesion, brain development, among others.

Conclusion: Based on our preliminary results, we suggest that different genetic pathways are involved in depressive phenotype in patients with HD. This information may lead to a better understanding of depression in $\mathrm{HD}$, a more accurate diagnosis, and more effective treatments.

Lay Summary: This study investigates the gene expression profile in HD patients with and without depression, and between subjects with HD and healthy controls. This information may help us better understand depression in HD and have a more accurate diagnosis, and more effective treatments.

14

Successful Living Kidney Donation in a Patient with Huntington's Disease

Christa S. Cooper ${ }^{1}$, Sarah M. Chen ${ }^{1}$, Bryan Bernard ${ }^{1}$, Jennifer G. Goldman $^{2}$
${ }^{1}$ Rush University Medical Center; ${ }^{2}$ Shirley Ryan AbilityLab and Northwestern University

Objective: To report a case of a Huntington's Disease (HD) patient who underwent living kidney donation and examine the decision-making process and ethical considerations of this.

Background: HD is an autosomal dominant, incurable, neurodegenerative disease with motor, cognitive, and psychiatric symptoms. According to UNOS, approximately 95,000 people within the United States await kidney donor. There are currently no published ethical or medical guidelines regarding kidney donation in persons with HD.

Methods: A 46-year old Caucasian male with 6 years of clinically manifest and genetically confirmed HD wished to donate his kidney to an unnamed recipient. He first expressed interest in altruistic kidney donation with the thought that "he wanted to help others." His clinical exam revealed moderate chorea, mildly impaired cognitive functions, and normal psychological functions. Discussions between the HD and transplant teams ensued as literature regarding this in HD is scant. Our patient underwent neuropsychological testing revealing mild deficits in executive functions, recent memory, and visual perception, consistent with mild cognitive impairment, and stable psychological function. After completing prerequisite testing (i.e., laboratory and urine tests, ECG, chest x-ray, and CTA abdomen and pelvis) by the HD and transplant teams, he was deemed eligible for kidney donation. He underwent laparoscopic nephrectomy 16 months after first inquiry.

Results: He tolerated the procedure well and had an excellent postoperative course. Follow up office visit one week post-op revealed discontinuation of pain medications and resumption of biking for transportation. His HD symptoms remained stable throughout hospitalization and post-operative course.

Conclusion: After undergoing the appropriate neurological, neuropsychological, and medical screening and pre-operative work-up, we believe a diagnosis of $\mathrm{HD}$ is not an absolute contraindication for kidney donation. A team approach and communication between the HD and transplant teams are essential. Decision-making capabilities, cognitive status, comorbid conditions, and support system must be taken into account.

Lay Summary: This is a case report of a Huntington's Disease patient who underwent living kidney donation and it examines the decisionmaking process. This case is important to the HD community because it will help to guide future decision making for HD patients that may be considering kidney donation as there are currently no published guidelines.

\section{5}

A New Screening Tool, the Huntington's Disease-Behavioral Questionnaire (HD-BQ), for Assessing Behavioral Disturbances in HD

Jody Corey-Bloom ${ }^{1}$, Sameer Aboufadel ${ }^{1}$, Ilex Beltran-Njaera ${ }^{2}$, Haileigh Smith $^{1}$, McKenna E. Williams ${ }^{2}$, Paul E. Gilbert ${ }^{2,3}$

${ }^{1}$ Department of Neurosciences, University of California San Diego, La Jolla, CA, USA; ${ }^{2}$ Department of Psychology, San Diego State University, San Diego, CA, USA; ${ }^{3}$ San Diego State University/University of California San Diego Joint Doctoral Program in Clinical Psychology, San Diego/La Jolla, CA, USA

Objective: To examine the usefulness of a new behavioral questionnaire for patients with, and at risk for, Huntington's disease (HD).

Background: Behavioral changes, including apathy, depression, irritability, anxiety, disinhibition, and difficulty prioritizing and initiating activities, are characteristic features of HD. For families and caregivers, they are often the most distressing aspect of the disease and their assessment is therefore important.

Methods: The HD-BQ, Mini-Mental State Examination (MMSE), Montreal Cognitive Assessment (MoCA), Unified Huntington's Disease 
Rating Scale (UHDRS) Total Functional Capacity (TFC) and Total Motor Score (TMS), Problem Behaviors Assessment-Short (PBA-s), and Hospital Anxiety and Depression Scale (HADS) were administered to $98 \mathrm{HD}, 68$ pre-manifest HD (PM), and 106 control (NC) subjects. The HD-BQ was also administered to $55 \mathrm{HD}$ caregivers.

Results: The HD-BQ discriminated between HD (mean 31.4, range 079), PM (mean 22.7, range 0-73) and NC (mean 15.3, range 0-65) subjects $(p<.001)$. Importantly, the HD-BQ identified significant differences between PM and NCs $(p=.048)$ that traditional cognitive ( $\operatorname{SDM} p=0.33$; MoCA $p=0.43$; MMSE $p=0.27$ ), functional (TFC $p=0.83$ ), and motoric (TMS $p=0.09$ ) measures did not. Effect sizes, measured by Cohen's $\mathrm{D}$, were also $60 \%$ greater for the HD-BQ $(\mathrm{d}=0.93)$ as compared to the HADS $(\mathrm{d}=0.58)$ and PBA-s $(\mathrm{d}=0.58)$ for NC vs HD subjects. Questionnaires completed independently by $55 \mathrm{HD}$ subject/caregiver pairs revealed a discrepancy in the perception of impairment between patients and their caregivers, with caregivers identifying significantly more behavioral changes (mean difference $=14.9$ points; $p<.001$ ).

Conclusions: The HD-BQ is a quick, reliable instrument for screening behavioral changes in individuals with, and transitioning to, HD. Behavioral changes are a possible early marker for HD onset. When possible, information regarding behavior should be corroborated by an informant since HD patients tend to underestimate their impairment.

Lay Summary: The objective of this study was to examine the usefulness of a new behavioral questionnaire for HD. We found that the HD-BQ revealed significant differences between HD subjects, premanifest HD subjects, and normal controls. We also found that HD subjects tend to underestimate their impairment.

16

A Novel Huntington's Disease Mouse Model to Assess the Role of Neuroinflammation on Disease Progression

Heather Dalenburg ${ }^{1}$, Angelica Bachman ${ }^{1}$, Haley Nelson ${ }^{1}$, Kari Pollock ${ }^{1}$, Peter Deng ${ }^{1,2,3}$, David Cameron ${ }^{1,2}$, Joseph Anderson ${ }^{1}$, Jan Nolta ${ }^{1}$, Kyle Fink $^{1,2}$

${ }^{1}$ Stem Cell Program and Institute for Regenerative Cures, University of California Davis Health Systems, Sacramento, CA, USA; ${ }^{2}$ Department of Neurology, University of California Davis Health Systems, Sacramento, CA, USA; ${ }^{3}$ Genome Center, MIND Institute, and Biochemistry and Molecular Medicine, University of California, Davis, CA, USA

Huntington's disease (HD) is a fatal autosomal-dominant neurodegenerative disease that affects 1 in every 10,000 individuals in the United States. $\mathrm{HD}$ is caused by a trinucleotide CAG repeat expansion of the huntingtin gene (HTT), which results in the mutated huntingtin protein (HTT). Although the exact cellular function of HTT is still not fully understood, researchers have found it to be essential in embryonic development and that it controls levels of brain-derived neurotrophic factor (BDNF).

Our lab developed novel immune deficient HD mouse models for two commonly used lines, the YAC128 and R6/2 mice, to enable transplantation studies using engineered human cells in addition to studying the impact of the immune system on disease progression. The primary goal of this project was to evaluate these novel immune deficient HD mice (YACNSG and R6/2-NSG) using behavioral and histology studies to the immune competent parental mouse strains and to immune deficient mice that had engraftment of a human immune system.

We used flow cytometry to confirm the novel HD strains lacked functional immune cells and in vivo imaging to access human MSC retention compared to a commonly used immune deficient line, the NSG mouse. We found that YAC-NSG and R6/2-NSG were able to retain human MSCs for the same length of time as the NSG mice. We performed behavioral assessments starting at 3 months of age until a year old with monthly testing on the accelerod and in the open field. At 12 months, brains were isolated and evaluated using immunohistochemistry for striatal volume and HTT aggregate. These results suggest that the novel immune deficient mice could be good models for human stem-cell based therapies and that the immune system may play an important role in the pathology of HD.

Lay Summary: Our group has created two novel Huntington's disease mouse models that lack a functional immune system. These mice enable studies of the role of the immune system, allow for the evaluation of transplanted human cells without immune suppressive drugs, and can be engrafted with a functional human immune system to study the interaction of a human immune system to the human mutant protein in a mouse.

\section{7}

A Study on Huntington Disease in Cyprus Reveals a Probable Founder Effect and Limited Uptake of Predictive Testing Among Affected Families

Christiana A. Demetriou ${ }^{1-2}$, Alexandros Heraclides ${ }^{1}$, Chrysanthi Salafori $^{3}$, George A. Tanteles ${ }^{4}$, Kyproula Christodoulou ${ }^{3,5}$, Yiolanda Christou $^{2}$, Eleni Zamba-Papanicolaou ${ }^{2,3}$

${ }^{1}$ Department of Primary Care and Population Health, University of Nicosia Medical School, Nicosia, Cyprus; ${ }^{2}$ Neurology Clinic D, The Cyprus Institute of Neurology and Genetics, Nicosia, Cyprus; ${ }^{3}$ The Cyprus School of Molecular Medicine, The Cyprus Institute of Neurology and Genetics, Nicosia, Cyprus; ${ }^{4}$ Clinical Genetics Clinic, The Cyprus Institute of Neurology and Genetics, Nicosia, Cyprus; ${ }^{5}$ Neurogenetics Department, The Cyprus Institute of Neurology and Genetics, Nicosia, Cyprus

The aim of this study was to examine Huntington Disease (HD) epidemiology, clinical characteristics and genetic testing trends in Cyprus, an island in southern Europe with extensive western European colonization in the past.

All registered HD patients in the Cyprus Republic, since 1994, were included. Detailed pedigrees and clinical information were recorded and maps, showing the geographic distribution of HD, were constructed. Requests for genetic testing were also examined.

The project identified 58 clinically manifested cases, 55 of which belonged to 16 families of Cypriot origin concentrated in a confined geographical cluster in Southeast Cyprus. In 2015 prevalence of symptomatic HD was 4.64/100,000 population, while incidence was 0.12 / 100.000 person-years. Prevalence displayed a marked increase during the past 20 years. Disease characteristics of HD patients were similar to those reported in western European populations. Since 1994, a total of 229 tests were performed of which $50.2 \%$ were diagnostic, $47.6 \%$ were predictive and $2.2 \%$ were prenatal. Using prevalent cases and Conneally's $1: 5$ ratio, there are roughly 210 individuals in the Cypriot population who are potential carriers of the HTT mutation and only $54.2 \%$ of these potential carriers have sought predictive testing. Also, the heterozygotes in the Cypriot population are estimated to 120 and only $68.3 \%(n=82)$ of them have been identified through genetic testing. Lastly, $\sim 35.20 \%$ of all subjects carrying the mutation are currently symptomatic.

The similarity of HD characteristics, incidence and prevalence in Cyprus with those in western European populations, together with the geographical clustering observed, support the possibility for a relatively recent founder effect of HD in Cyprus, potentially of western European origin. Genetic testing trends demonstrate limited uptake of predictive testing among affected families, which becomes particularly important in light of the recent availability of prenatal genetic testing and discovery of huntingtin lowering therapies.

Lay Summary: This abstract describes the only study of the epidemiology of Huntington Disease in Cyprus, a genetically interesting population. In addition, it includes a detailed investigation of genetic testing uptake among affected families, demonstrating limited uptake of predictive testing. Local stakeholders need to address this issue since it becomes of extreme importance as prenatal genetic diagnosis becomes more popular and as huntingtin lowering therapies are being discovered and approved. 
18

Novel Cell-Based Delivery Platform for DNA-Binding Domains Designed to Repress Mutant Huntingtin

Peter Deng ${ }^{1,2,3}$, Julian Halmai ${ }^{1,2}$, Nicole Coggins ${ }^{3}$, Jennifer Waldo ${ }^{1,2}$, Jasmine Carter ${ }^{1,2}$, Casiana Gonzales ${ }^{1,2,4}$, Krista Thongphanh ${ }^{1,2}$, Samantha Del Campo ${ }^{1,2}$, David Cameron ${ }^{1,2}$, Jan Nolta ${ }^{1}$, David Segal ${ }^{3}$, Kyle Fink ${ }^{1,2}$

${ }^{1}$ Stem Cell Program and Institute for Regenerative Cures, University of California Davis Health Systems, Sacramento, CA, USA; ${ }^{2}$ Department of Neurology, University of California Davis Health Systems, Sacramento, CA, USA; ${ }^{3}$ Genome Center, MIND Institute, and Biochemistry and Molecular Medicine, University of California, Davis, CA, USA; ${ }^{4}$ Department of Biological Sciences, California State University, Humboldt, CA, USA

The advent of nuclease-deficient DNA binding domains (DBDs) such as zinc fingers and CRISPR/dCas9 for tunable gene expression have revolutionized approaches towards treating genetically-linked neurologic disorders such as Huntington's Disease. An effective delivery system that can traffic these proteins to target tissue remains a key barrier for translating gene modifying DBDs into the clinic. Cell-based delivery methods, such as mesenchymal stem cells (MSC), are an attractive delivery vehicle for DBDs since such cells can be readily reprogrammed to secrete large proteins and are not limited in their transgene packaging size unlike adeno-associated viral approaches. To evaluate this system, we have engineered bonemarrow derived MSCs to act as in-vivo biofactories that secrete DBDs into the extracellular space whereby the DBDs are uptaken by neighboring cells, and shuttled into the nucleus to alter gene expression.

Previously, MSC-DBD proof-of-concept studies were evaluated with lentiviral-engineered bone marrow-derived MSCs that secrete a zinc finger protein to reverse a neuronal imprinting event in a neuronal reporter mouse model. We observed significant reversal of the imprinting event via IHC and western blotting for a fluorescent reporter (YFP) in the hippocampus, cerebellum, and cortex as compared to control treatment groups 3- and 6-weeks post-intracranial transplantation of MSC-DBDs. Presently, we have adapted our MSC-DBD system for the secretion of larger DBDs such as transcription activator-like effectors and dCas9 protein fused with a repressive domains as silencing approaches towards Huntington's Disease. In the current study, we evaluate the secretion, distribution, and uptake of secreted DBDs in both in-vitro and in-vivo models for HD. We report the first-of-its-kind use of MSCs as a delivery platform for gene modifiers in neurologic disease - expanding the range of gene delivery vehicles in the field.

Lay Summary: A major barrier in advancing promising gene therapeutics into the clinic are effective delivery systems for large gene modifying proteins. The utilization of a mesenchymal stem cell based system may prove advantageous in this regard since they are able to package very large proteins and can further act as a biofactory for protein production once transplanted into the body. These secreted proteins can then be uptaken by neighboring cells such as neurons where a gene modifying event can occur in a therapeutically relevant manner.

19

\section{Cognitive Interference of Gait Initiation in Huntington's Disease}

Radhika Desai, Lori Quinn

Teachers College, Columbia University, New York, NY, USA (all authors)

Background: Gait initiation encompasses the preparation and execution of the first step of a gait sequence, and requires appropriate motor planning integration and scaling. Cognitive loads have the ability to interfere with both the scaling and timing of gait initiation. However, deficits in duration and magnitudes of gait initiation, along with related cognitive interference patterns, have not yet been identified in individuals with HD.

Methods: 48 participants, including 33 individuals with manifest HD (20 males, 13 females, mean age $54.67 \pm 12.57$, mean total motor score (TMS): 11(2)), and 15 healthy controls ( 8 males, 7 females, mean age $53.2 \pm 13.18$ ) wore 3 APDM inertial sensors during a $14 \mathrm{~m}$ walk for a single task condition, and again during a cognitive load condition. During the cognitive load condition, participants walked and performed a verbal DKEFS task, alternating categorical fruits and furniture. A repeated measures 2-way ANOVA assessed magnitudes and durations of anticipatory postural adjustments (APAs) across groups and conditions.

Results: A significant main effect, $(F(1,46)=46.65, p<.00)$, was found between groups, among measures of APA acceleration amplitudes. Pairwise comparisons revealed significant differences $(p=.04)$ of acceleration amplitudes between No-Load and Cognitive-Load within the HD group. No significant differences were present among acceleration amplitudes for the control group between conditions. Furthermore, differences in durations between conditions were not present in both $\mathrm{HD}$ and controls.

Conclusions: Substantially large acceleration values characterize an impairment of APAs in individuals with HD. Impaired scaling of accelerations are worsened when in the presence of a cognitive load in HD. In contrast, durations of APAs are preserved across groups and conditions, revealing a discrepancy in movement scaling in HD. Additional research is needed to identify the neural correlates behind motor planning and scaling in HD.

Lay Summary: Components of gait initiation are susceptible to cognitive load and reflect motor planning integrity. HD groups exhibited larger magnitudes of APA accelerations across conditions but maintained durations. This atypical time-scaling pattern provides a behavioral window into motor planning deficits in individuals with HD.

\section{0}

Patient and Close-other Reported Discourse Deficits at Varying Stages of Progression in Huntington's Disease

Sarah K. Diehl ${ }^{1}$, Zachary DeWall ${ }^{2}$, Mary Dietrich ${ }^{1}$, Daniel O. Claassen ${ }^{2}$, Melissa C. Duff ${ }^{2}$, Michael de Riesthal ${ }^{2}$

${ }^{1}$ Vanderbilt University, Nashville, TN, USA; ${ }^{2}$ Vanderbilt University Medical Center, Nashville, TN, USA

Huntington's disease (HD) is an inherited neurodegenerative disorder characterized by chorea, cognitive decline, and personality changes. Average onset occurs at 35 to 40 years of age with progression to death approximately 10 to 20 years following diagnosis (Caviness, 2000). Little research exists to educate clinicians about discourse, or cognitive communication deficits, in HD. The current study aims to characterize the cognitive communication deficits that impact discourse abilities in people with HD using the La Trobe Communication Questionnaire (LCQ) "self" and "other" forms. Participants included a total of 60 patients with HD across 4 stages of disease progression and 60 caregivers or close others. Although variable, results suggest that patients and close others report discourse impairments at the total score and domain levels of the LCQ. As expected, a greater level of deficit is reported by patients and close others in later disease stages. However, cases present with reported deficits as early as the pre-manifest stage (prior to motor symptom onset). No significant difference was found between LCQSelf and LCQ-Other total scores; however, heterogeneity is observed in individual patient-close other pairs. Findings will be discussed related to additional clinical variables including measures of disease severity and cognitive ability.

Lay Summary: Cognitive impairment may present as an early symptom of Huntington's disease and negatively impact patients' ability to communicate during everyday interactions. This exploratory study describes the patient and caregiver reported discourse impairments across disease progression. 
21

Genetic Modulators of 3-Nitropropionic Acid Neurotoxicity: Implications for Huntington's Disease

Paula Dietrich, Shanta Alli, Megan Mulligan, Robert Williams, Ioannis Dragatsis

The University of Tennessee, Health Science Center, Memphis, TN (all authors)

Although several genetic animal models of Huntington's Disease (HD) have been generated and analyzed over the past decades, none of them recapitulate the striatal neurodegeneration typical of adult-onset HD. On the other hand, systemic administration of the mitochondrial toxin 3nitropropionic acid (3NP), an irreversible inhibitor of complex II succinate dehydrogenase $(\mathrm{SDH})$, induce striatal-specific degeneration in primates and rodents. $3 \mathrm{NP}$ models have been validated by several research groups as reliable models for HD, since they recapitulate not only the striatal neurodegeneration but also several other phenotypic and biochemical features characteristic of the disease. Notably, while the mechanisms underlying preferential striatal vulnerability and degeneration in HD are still unknown, biochemical studies in HD brain tissue and in HD cellular models indicate that mitochondrial dysfunction, and in particular complex II inhibition, plays an important role in HD pathogenesis.

We have found that systemic administration of $3 \mathrm{NP}$ induces extensive striatal lesions in the $\mathrm{C} 57 \mathrm{BL} / 6 \mathrm{~J}$ mouse strain, while the DBA/2 J mouse strain is completely resistant and shows no signs of striatal damage. Using recombinant inbred BXD (C57BL/6 J X DBA/2 J) strains, we identified a $2 \mathrm{MB}$ resistance locus in chromosome 7 . This region contains 15 genes, and among them, Ccnd1, the gene encoding Cyclin D1, a major effector of cell cycle re-entry. Our preliminary results indicate that Cyclin D1 is differentially activated in the striatum of $\mathrm{C} 57 \mathrm{BL} / 6 \mathrm{~J}$ and DBA/2 J parental mouse strains in response to $3 \mathrm{NP}$, suggesting a direct link between cell cycle re-entry and cell death. Consistent with this hypothesis, evidence of aberrant cell cycle reactivation has been observed in several models of neurodegenerative diseases, as well as in HD post-mortem brain. Further analyses of the role of Cyclin D1 and cell cycle re-entry in 3NP-induced neurodegeneration may therefore pave the way for the development of novel therapeutic strategies for HD and other neurodegenerative disorders.

Lay Summary: Our study aims to identify genetic factors that confer resistance to striatal neuronal cell death in a non-genetic mouse model of HD. Our findings may lead to the identification of genetic modulators of HD and pave the way to the development of new and more effective therapeutic treatments for HD and other neurodegenerative disorders.

\section{2}

Prevalence of Huntington's Disease in a Small City in the State of Alagoas, Brazil

Valmir dos Santos Júnior ${ }^{1,2}$, Maria Aparecida Santos de Souza Alencar ${ }^{3}$ ${ }^{1}$ Information Technology Course Federal Institute of Alagoas - IFAL Arapiraca AL - Brazil; ${ }^{2}$ Huntington Youth Association of Brazil (ABH Jovem); ${ }^{3}$ Huntington Association of Brazil (ABH)

Huntington's Disease (HD) is an autosomal dominant neurodegenerative disorder caused by an expansion in the CAG trinucleotide in HTT gene responsible for coding a protein called huntingtin. In Feira Grande, a small town in northeastern of Brazil, HD was described for the first time in 2010 .

Objectives: The goal of this study was to investigate the HD prevalence in Feira Grande town. It was the only study done after 8 years since the first HD case was reported in this region.

Methodology: This research was exploratory and with quantitative approach. A semi-structured questionnaire (with quantitative approach) was applied during meetings to get some epidemiological data from health professionals and government managers of this town.

Results: Until May 2018, 23 individuals were reported as HD affected in Feira Grande. And $10 \mathrm{HD}$ affected individuals immigrated to other regions looking for caregivers or other reasons. There are more than 200 individuals at HD risk. Therefore, the prevalence of Huntington's disease in this town is 10.4 cases per 10,000, higher than in European countries with 1/10,000.

Conclusions: Feira Grande town, in the state of Alagoas, currently has the highest prevalence of Huntington's disease in Brazil, already reported, when compared to the other two known clusters: in Ervália-MG and in Sobral-CE. The HD families have scarce support of government. They do not have multidisciplinary assistance and HD information. Therefore, this study is important to collaborate with researchers about the HD epidemiology in Brazil.

Lay Summary: This study is important to collaborate with researchers about the HD epidemiology in Brazil.

\section{3}

Genetic Testing for Huntington's Disease: One Center's Experience and Lessons Learned

Erin Furr Stimming, Raja Mehanna, Maria Rossetti, Amber McCarthy UT Health McGovern Medical School, Houston, TX, USA (all authors)

There is increasing demand and interest from at-risk and symptomatic individuals for molecular testing for Huntington's disease (HD). This has been spurred by recent advances in HD clinical research including the investigation of potential disease-modifying agents. The Huntington's Disease Society of America (HDSA) provides a genetic testing protocol for Huntington's disease which is utilized by clinics nationwide. Implementation of this protocol relies on a clinical team of specialists including genetic counselors, neurologists, mental health professionals and social workers. Here we report on the first three years of a rapidly growing Huntington's disease genetic testing program. We outline current practices in a clinic that has more than quadrupled in size since inception. We discuss challenges to successful adherence to the HDSA guidelines and strategies for overcoming these barriers. We evaluate the evolving landscape of HD treatment and its implications for genetic counseling and genetic testing. We call for applying lessons learned in the genetic testing of people with Huntington's disease to similar diseases.

Lay Summary: We report on experience of genetic testing over the past few years for Huntington's Disease. We identify a trend in an increase in the uptake of and interest in diagnostic and/or predictive genetic testing.

\section{4}

Percutaneous Endoscopic Gastronomy Tubes in Huntington's Disease

Allison Dyke ${ }^{1}$, Samuel Frank ${ }^{2}$

${ }^{1}$ University of New Hampshire at Manchester, Manchester, NH, USA; ${ }^{2}$ Beth Israel Deaconess Medical Center, Boston, MA, USA

Background: Huntington's disease (HD) is a fatal hereditary neurodegenerative disorder characterized by progressive motor and cognitive symptoms. When swallowing is impacted, percutaneous endoscopic gastronomies (PEG) tubes are often recommended. However, the impact of PEG tubes in HD is unknown.

Methods: A retrospective chart review of living and deceased HD patients was conducted to examine whether PEG tubes impact pneumonia risk, length of life, weight, serological nutritional measures or skin issues. The groups with and without PEG placement were compared. For between-groups analyses independent samples t-tests and chi-square test for independence were performed. For within-group analyses pairedsamples t-tests were performed. 
Results: Records of 39 patients who had a PEG inserted and 109 who did not were examined. The mean age of patients living with HD was $58.3 \pm$ 12.7 years and deceased $57.7 \pm 10.3$ years. At the time of analysis, the mean duration of HD was $14.2 \pm 7$ years. Groups were similar in gender, age, and weight at admission. In those deceased $(n=62)$, insertion of a PEG tube increased length of life with $\mathrm{HD}(16.8 \pm 6.7$ vs. $13.2 \pm 4.9$ years, $p=0.02)$. Other measures impacted by PEG placement were reduced cholesterol levels $(172.2 \pm 34.1$ vs. $150.5 \pm 23.4, p=0.05)$, increased prevalence of skin ulcers $(44 \%$ vs. $12 \%, p<0.001)$ and increased rate of pneumonia $(77 \%$ vs. $35 \%, \mathrm{p}<0.001)$. Insertion of a PEG tube did not significantly change weight or albumin levels.

Conclusions: PEG tube placement in advanced HD provided benefit in length of life, but weight, nutritional measures and the rate of pneumonia were either not impacted or made worse with the insertion of a PEG tube. The impact on quality of the extended life needs further study, but providers, patients and families should consider all options when discussing preferences for interventions.

Lay Summary: To date there is no study that evaluates the risk of developing pneumonia after the insertion of a PEG feeding tube specifically in HD patients. This study examined whether PEG feeding altered the risk of developing aspiration pneumonia, prolonged life, impacted weight or serological nutritional measures and changed the rate of skin issues in HD patients. Results of this study are important to the HD community, as they illustrate the effectiveness of PEG feeding tubes in the HD population, which can allow families and patients to make informed decisions regarding their treatment plans.

\section{5}

Composite UHDRS Shows Extensive Spatial Correlation with Gray Matter and White Matter Volume in Huntington's Disease Gene Carriers

C Estevez-Fraga ${ }^{1}$, EB Johnson ${ }^{1}$, FB Rodrigues ${ }^{1}$, P Zeun ${ }^{1}$, A Durr ${ }^{2}$, B Leavitt $^{3}$, RAC Roos ${ }^{4}$, DR Langbehn ${ }^{5}$, S Gregory ${ }^{1}$, G Rees ${ }^{1}$, RI Scahill ${ }^{1}$, SJ Tabrizi ${ }^{1}$, and the TrackHD investigators

${ }^{1}$ UCL Institute of Neurology, London, UK; ${ }^{2}$ Pitié-Salpêtrière Hôpital, Paris, France; ${ }^{3}$ UBC, Vancouver, Canada; ${ }^{4}$ Leiden University Medical Centre, Leiden, Netherlands; ${ }^{5}$ Carver College of Medicine, Iowa City, IA, USA

Objective: This work sought to determine the structural correlates of variability in cUHDRS in HD gene expansion carriers (HDGC).

Background: Symbol digit modalities test (SDMT), Stroop wordreading test and Total Motor Score (TMS) are the most reliable clinical measures in Huntington's disease (HD). Recently a composite measure of motor, cognitive and functional outcome (cUHDRS) has been proposed as a sensitive clinical tool in early $\operatorname{HD}(1)$.

Methods: Participants were recruited from the TRACK-HD study which included 120 premanifest (preHD) and 123 early HD patients. Clinical assessments were performed at four sites. 3 T MRI data were acquired according to a standardized protocol. Nine participants were excluded from the analysis due to poor quality scan or incomplete clinical assessments leaving a total of 234 HDGCs (118 preHD and 116 early HD). Correlation analysis was performed using voxel-based morphometry (VBM) in SPM12. We studied the correlation between SDMT, Stroop, TMS and cUHDRS scores with gray matter $(\mathrm{GM})$ and white matter (WM) volumes at baseline. Results were considered significant at cluster-level $p<0.05$ (FWE-corrected).

Results: Clinical impairment in HDGC was correlated with GM volume in caudate and putamen as well as with surrounding WM volume. This correlation between cUHDRS and both GM and WM volumes was spatially extensive and statistically significant.

Conclusion: In HDGCs, cUHDRS shows a significant correlation with GM and WM volumes in a fashion that extends throughout the basal ganglia and surrounding WM, suggesting this composite clinical measure links closely with underlying HD pathology.
1. Schobel SA, Palermo G, Auinger P, Long JD, Ma S, Khwaja OS, et al. Motor, cognitive, and functional declines contribute to a single progressive factor in early HD. Neurology. 2017;89(24):2495-502.

Lay Summary: Composite UHDRS is the principal outcome (in the EU) of the phase 3 trial with antisense oligonucleotides. This research shows which areas of the brain are correlated with this scale. It shows an extensive association between the scale and both white and gray matter when compared to other established scales.

\section{6}

Correlation Between Structural Neuroimaging and Clinical Outcomes in a Small Sample of Patients with Early Huntington's Disease

Carlos Estevez-Fraga ${ }^{1}$, Eileanoir B Johnson ${ }^{1}$, Filipe B Rodrigues ${ }^{1}$, Paul Zeun ${ }^{1}$, Alexandra Durr ${ }^{2}$, Elin M Rees ${ }^{3}$, Nicola Z Hobbs ${ }^{3}$, Raymund AC Roos $^{4}$, Bernhard Landwehrmeyer ${ }^{5}$, Sarah Gregory ${ }^{1}$, Rachael I Scahill ${ }^{1}$, Geraint Rees ${ }^{1}$, Sarah J Tabrizi ${ }^{1}$

${ }^{1}$ UCL Institute of Neurology, London, UK; ${ }^{2}$ Pitié Salpêtrière Hôpital, Paris, France; ${ }^{3}$ Ixico, London, UK; ${ }^{4}$ Leiden University Medical Centre, Leiden, The Netherlands; ${ }^{5}$ University of Ulm, Ulm, Germany

Objective: Here we sought to correlate the best clinical measures in $\mathrm{HD}$ with atrophy rates and brain volumes in a small sample of early manifest patients. Background: Scores on the symbol digit modalities test (SDMT), Stroop word-reading, Total Motor Score (TMS) and composite UHDRS (cUHDRS) are correlated with disease progression and brain changes in large samples of patients with Huntington's disease (HD).

Methods: Participants were recruited from the PADDINGTON study and comprised 49 early HD patients from 4 sites.

Statistical analysis was performed using Stata version 12.0. The boundary-shift integral (BSI) was used to calculate change over 15 months in whole brain, caudate and ventricular volumes. Baseline gray matter (GM), white matter (WM), whole-brain (WB) volumes and voxelbased morphometry (VBM) analysis were performed using SPM12. VBM results were considered significant at $p<0.001$.

Results: Ventricular BSI correlated with SDMT $(p=0.038)$, Stroop $(p=$ $0.005)$, TMS $(p=0.026)$ and cUHDRS $(p=0.006)$. GM volume showed statistically significant correlations with SDMT $(\mathrm{p}<0.001)$, Stroop $(\mathrm{p}=$ $0.03)$, TMS $(p=0.001)$ and cUHDRS $(p=0.045)$. WB volume correlated with SDMT $(p<0.001)$, Stroop $(p=0.045)$, TMS $(p=0.02)$. Brain BSI showed correlations with Stroop $(p=0.03)$ and cUHDRS $(p=0.03)$, all results shown at an uncorrected level.

VBM showed correlations between baseline GM volumes of several different subcortical nuclei with all clinical measures.

Conclusion: There were statistically significant associations between clinical measures and change over time in brain volumes, suggesting that clinical decline is closely linked to ongoing pathology in HD.

Lay Summary: This is important to the HD community because it shows that even in a small sample of patients with early HD we can see a significant association between different brain areas and clinical scales. This might mean that if one drug has an impact in the scale it may be associated with reduced brain loss.

\section{7}

Dual-Task Performance During Standing Balance and Walking in Persons with Huntington's Disease

Lori Quinn ${ }^{1}$, Greg Youdan ${ }^{1}$, Lisa Muratori ${ }^{2}$, Xueyao Li $^{1}$, Nora Fritz ${ }^{3}$ ${ }^{1}$ Teachers College, Columbia University, New York, NY, USA; ${ }^{2}$ Stony Brook University, Stony Brook, NY, USA; ${ }^{3}$ Wayne State University, Detroit, MI, USA

Background: Individuals with Huntington disease (HD) experience declines in both motor and cognitive function, and these impairments result 
in difficulty performing motor and cognitive tasks simultaneously which may contribute to fall risk. Little is known about performance of individuals with HD on motor-cognitive dual-tasks or the relationship of clinical dual-task measures with disease specific measures of disability, cognitive function, and reports of falls.

Methods: Forty-three individuals with HD (mean age: 53.6 years; mean TMS: 40.6) and 15 healthy controls (mean age: 52.2 years) completed $7 \mathrm{~m}$ walk test, standing balance, seated cognitive performance (Stroop, Symbol Digit Modalities Test (SDMT), Delis-Kaplan Executive Function System (DKEFS)) and dual-task performance while standing (+ Stroop) and walking (+ DKEFS). Mann-Whitney tests were used to assess between-group differences, correlations to clinical measures were assessed using Spearman correlations, and Fisher $\mathrm{r}-$ to- $\mathrm{z}$ transformations were used to compare correlations. Results: Individuals with HD performed worse than healthy controls on both cognitive and motor dual-tasks in both standing (greater sway in Standing+Stroop $(\mathrm{p}<0.001))$ and while walking (slower walking $(p=$ $0.003)$ ), and had poorer executive function (lower DKEFS $(\mathrm{p}<0.001)$ ). Within the HD group, cognitive dual-task performance during standing was more strongly associated with disease-specific measures than dualtask during walking (TMS: $p=0.0042$, TFC: $p=0.022$ ). Motor dual-task performance during both standing and walking was also correlated with TMS (Walking+DKEFS: $r=-0.42$; Standing+Stroop: $r=0.64$ ). Better motor dual-task performance was significantly correlated with better performance on the Stroop (Walking+DKEFS: $r=0.38$; Standing+Stroop: $r=$ -0.39 ) and SDMT (Standing+Stroop: $r=-0.58$ ) and was correlated with fewer reports of falls (Standing+Stroop: $r=0.51$ ) in the prior 6 months.

Conclusions: Standing dual-task performance may be a more sensitive measure of motor impairment than walking dual-task performance. Standing dual-task performance may be a contributing factor to falls and overall motor function, and a target of clinical intervention to address these impairments directly.

Lay Summary: Individuals with HD experience declines in motor and cognitive function, yet little is known about the ability of individuals with HD to perform motor and cognitive task simultaneously, and how this might relate to overall function and fall risk. This study shows that performing multiple tasks at once (dual-tasks) is related to both motor and cognitive function, and may be a contributing factor to falls. These results are important because they provide a critical target for clinical intervention to improve motor function and reduce fall risk.

\section{8}

\section{Novel PET Data and Analysis of Early HD from Pride-HD}

Ralf Reilmann ${ }^{1}$, Warren C. Olanow ${ }^{2}$, Mika Leinonen ${ }^{2}$, Andrew McGarry $^{2}$, Michal Geva ${ }^{3}$, Ferdinando Squitieri ${ }^{8}$, Frederic Saudou ${ }^{7}$, Lynn Raymond ${ }^{6}$, Igor Grachev ${ }^{5}$, Philipp M. Meyer ${ }^{4}$, Michael Hayden $^{3,6}$, Osama Sabri ${ }^{4}$, Karl Kieburtz ${ }^{2}$

${ }^{1}$ George-Huntington-Institute, Technology-Park, Muenster, Germany; ${ }^{2}$ Clintrex LLC, Sarasota, Florida, USA; ${ }^{3}$ Prilenia Therapeutics, Herzliya, Israel; ${ }^{4}$ University Hospital of Leipzig, Department of Nuclear Medicine, Leipzig, Germany; ${ }^{5}$ Guide Pharmaceutical Consulting, LLC, NJ, USA; ${ }^{6} \mathrm{CMMT}$, UBC, Canada; ${ }^{7}$ Univ. Grenoble Alpes, INSERM, CHU Grenoble Alpes, Grenoble Institute Neurosciences, Grenoble, France; ${ }^{8}$ IRCCS Neuromed, Pozzilli, Italy

Pridopidine is a selective Sigma-1 Receptor (S1R) agonist. S1R regulates key cellular pathways commonly impaired in neurodegenerative diseases (NDD) including BDNF secretion, calcium signaling and mitochondrial function. BDNF is a key protein promoting neuronal plasticity and survival and is reduced in brains of HD patients and animal models. Overexpression of BDNF alleviates numerous disease features in the YAC128 HD mice. Pridopidine upregulates the BDNF pathway in multiple NDD models. It increases BDNF protein levels (6-OHDA PD mice and rat neuroblastoma cell-lines) and rescues the impaired BDNF axonal transport in HD neurons. Pridopidine upregulates BDNF-TrkB downstream signaling, including p-ERK (6-OHDA mice, HD neurons) and CREB (YAC128 mice). Furthermore, pridopidine shows dose dependent neuroprotection in human HD iPSCs, and mice HD neurons, and restores homeostatic plasticity in HD cortical neurons.

In humans, PET imaging confirmed the $45 \mathrm{mg}$ BID dose to have a full $(>90 \%)$ and selective S1R target engagement ( $\sim 3 \%$ D2/3Rs occupancy). The Pride-HD trial assessed pridopidine for 52 weeks with Total Motor Score (TMS) as a primary outcome, and Total Functional Capacity (TFC) as a pre-specified secondary measure. Q-motor was an exploratory unbiased measure of motor function.

While all doses had some positive effect, the maximal effect (less TFC decline) was seen with $45 \mathrm{mg}$ BID at week $52(0.87 \Delta$ from placebo, $p=$ 0.0032 ). Post-hoc analysis showed this effect is most prominent in early HD patients (HD1 + HD2) (1.16 $\Delta$ from placebo, $p=0.0003)$. While TMS showed a positive trend, it was not met due to high placebo effect. Q-motor, a placebo independent assessment, provides support for motor effect of pridopidine. Multiple Q-motor measures were improved already at 12 weeks in all HD, with supported efficacy in the early HD sub-group. These data support further investigation of pridopidine on TFC in HD.

Lay Summary: We present here new, unpublished data demonstrating pridopidine effect on TFC and Q-motor in early HD patients. Pridopidine represents a novel moa for the treatment of HD patients, which was not understood previously, resulting in previous wrong clinical design. This is of high important to the HD community as a first drug showing positive effect on TFC in HD clinical trials.

\section{9}

Pridopidine, a Highly Selective S1R Agonist for the Treatment of Huntington Disease

Michal Geva ${ }^{1}$, Lynn Raymond ${ }^{2}$, Chris Ross ${ }^{3}$, Ana Cristina Rego ${ }^{4}$, Frederic Saudou ${ }^{5}$, Ilya Bezprozvanny ${ }^{6}$, Michael Hayden ${ }^{1,2}$

${ }^{1}$ Prilenia Therapeutics, Herzliya, Israel; ${ }^{2}$ CMMT, UBC, Canada; ${ }^{3}$ John's Hopkins University, USA; ${ }^{4} \mathrm{CNC}-\mathrm{Center}$ for Neuroscience and Cell Biology, University of Coimbra, Coimbra, Portugal; ${ }^{5}$ Univ. Grenoble Alpes, INSERM, CHU Grenoble Alpes, Grenoble Institute Neurosciences, Grenoble, France; ${ }^{6}$ UT Southwestern Medical Center at Dallas, USA

Pridopidine is a selective Sigma-1 receptor (S1R) agonist. The S1R is an intracellular protein located mainly at the Mitochondria Associated Membranes (MAM), modulating diverse cellular processes including calcium signaling, mitochondrial function and BDNF levels. Importantly, loss of function mutations in the S1R are associated with neurodegenerative diseases, while S1R activation exerts neuroprotective effects.

Pridopidine demonstrates neuroprotective effects which are mediated via the S1R in different preclinical models of neurodegeneration, including HD and AD. Pridopidine rescues the loss of dendritic spines in HD YAC128 striatal neurons, and the loss of mushroom spines in AD PS1-KI and APP-KI hippocampal neurons. Pridopidine also normalizes calcium-signaling abnormalities observed in HD (YAC128) and AD (PS1-KI and APP-KI) neuronal cultures. S1R knock-out (KO) or inhibition, using known S1R antagonists abolishes pridopidine effects.

Pridopidine exhibits a robust and dose dependent neuroprotective effect against mHTT-induced cell death in human HD iPSCs, and in mouse HD neurons. In HD cortico-striatal cultures, pridopidine rescues BDNF axonal transport, synaptic activity and increases phosphorylation of the pro-survival ERK protein. Pridopidine restores mitochondrial function in YAC128 neurons and in human HD neuronal stem cells as measured by increased ATP production, respiration and mitochondrial membrane potential. Furthermore, pridopidine enhances homeostatic plasticity, a key mechanism for maintaining synaptic stability and strength shown to be impaired in 
HD. This is thought to be important for maintaining cognitive functions that are impaired early in HD.

Clinical data supports S1R as the primary target of pridopidine. Pridopidine $45 \mathrm{mg}$ bid dose is shown to have full (>90\%) and selective occupancy of the S1R in healthy volunteers and HD patients (PET imaging, unpublished data). In the PRIDE-HD trial, $45 \mathrm{mg}$ bid pridopidine demonstrated a significant effect in maintaining Total Functional Capacity (TFC, an accepted endpoint measuring disease stage) after 1year treatment, indicating functional benefit.

Lay Summary: Pridopidine represents a drug in clinical development with novel moa for the treatment of HD. We present here compelling preclinical data demonstrating neuroprotective effects of pridopidine in models of HD and other neurodegenerative disorders. Pridopidine is in clinical development, and a phase 3 trial in HD is planned to be initiated in the near future.

\section{0}

Pridopidine Rescues Multiple Mitochondrial Functions Impaired in Human and Mouse HD Models by Activation of the Sigma-1 Receptor

Michal Geva ${ }^{1}$, Luana Naia ${ }^{2}$, Philip Ly ${ }^{3}$, Carla Lopes ${ }^{2}$, Sandra I. Mota ${ }^{2}$, Carina Maranga ${ }^{2}$, Michael Hayden ${ }^{1,3}$, A. Cristina Rego ${ }^{2,4}$

${ }^{1}$ Prilenia Therapeutics, Herzliya, Israel; ${ }^{2} \mathrm{CNC}$-Center for Neuroscience and Cell Biology, University of Coimbra, Coimbra, Portugal; ${ }^{3}$ Centre for Molecular Medicine and Therapeutics, BC Children's Hospital Research Institute, University of British Columbia, Vancouver, British Columbia, Canada; ${ }^{4}$ FMUC-Faculty of Medicine, University of Coimbra, Coimbra, Portugal

Pridopidine demonstrates neuroprotective functions in several preclinical models of neurodegenerative disorders, including enhancement of neuronal plasticity, axonal transport, and upregulation of neurotrophic factors, via activation of the Sigma-1 receptor (S1R). The S1R is a chaperone protein localized in the mitochondriaassociated endoplasmic reticulum (ER) membranes (MAM), where it regulates ER-mitochondrial $\mathrm{Ca}^{2+}$ signaling, generation of reactive oxygen species (ROS) and mitochondrial fission which features are disturbed in Huntington disease (HD). Here, we investigated the protective effect of pridopidine on various mitochondrial functions in human and mouse HD models. Using primary neurons from YAC128 HD mice, expressing the mutant human HTT gene, we observed that pridopidine enhances mitochondria-ER tethering while improving mitochondrial activity, elongation and motility. A similar increase on mitochondrial respiration was observed in human HD neural stem cells (NSCs) treated with pridopidine. Increased susceptibility to oxidative challenge was observed in HD mouse neurons, human HD NSCs and in human HD lymphoblasts, resulting in increased levels of ROS and deficient antioxidant response. Pridopidine treatment rescues the antioxidant response and reduces ROS levels in all three HD models. These protective effects of pridopidine were abolished after knock down of the S1R. The protective effect of pridopidine was further confirmed in-vivo, in YAC128 HD mice treated with pridopidine before the appearance of the first symptoms. In-vivo, pridopidine reduced mitochondrial ROS levels through regularized mitochondrial complexes activity, accompanied by a significant recovery of motor coordination. These data indicate pridopidine exerts neuroprotective activities, by enhancing the impaired mitochondrial function in HD, via activation of the S1R. Restoration of mitochondrial function represents a mechanism by which pridopidine exerts its neuroprotective effects.

Lay Summary: This abstract provides data to support mechanistic and protective effects in HD human and mouse models, for a drug in clinical development for HD. This is unpublished data, presenting profound preclinical data suggesting a therapeutic effect for HD patients.
31

Discovering Dominant Features Associated with Disease Progression in Huntington's Disease: A Data-Driven Approach Using the EnrollHD Database

Naghmeh Ghazaleh ${ }^{1}$, Richard Houghton ${ }^{1}$, Giuseppe Palermo ${ }^{1}$, Scott Schobel $^{1}$, Peter A. Wijeratne ${ }^{2}$ Jeffery D. Long ${ }^{3,4}$

${ }^{1}$ F. Hoffmann-La Roche Ltd., Basel, Switzerland; ${ }^{2}$ Centre for Medical Imaging Computing, Department of Computer Science, University College London, London, UK; ${ }^{3}$ Department of Psychiatry, University of Iowa, Iowa City, USA; ${ }^{4}$ Department of Biostatistics, University of Iowa, Iowa City, USA

This study systematically tested and ranked the statistical importance of potential prognostic factors on different aspects of disease progression in manifest Huntington's Disease (HD; Stages I-V). Enroll-HD registry data from participants with adult-onset HD, aged 25-65 years, Independence Scale $\geq 70$ at baseline and $\geq 2$ annual visits were used in the analysis $(N=$ 1608; CAG repeats: 36-64). We considered 103 features for each participant, including demographics, clinical characteristics, comorbidities, symptoms, pharmacological and non-pharmacological treatments and Independence Scale at baseline. A random forest-based algorithm was used to rank the features in terms of their ability to predict outcomes (using an importance measure called the permutation importance accuracy). The outcome measures for each patient were their estimated 2-year change from baseline in the composite Unified Huntington's Disease Rating Scale (cUHDRS), and 2-year change in the component scores: Total Motor Score (TMS), Single Digit Modality Test (SDMT), Stroop Word Reading Test (SWRT) and Total Functional Capacity (TFC).

CAP (CAG-age product) ranked as the first most predictive feature of progression for all outcomes and CAG as the second for all except SWRT, where Independence Scale ranked as second and CAG as third. Independence Scale ranked third for SDMT, TMS and cUHDRS and eighth for TFC. Other features associated with faster progression that ranked in the top 10 for at least three of the five outcomes were: being accompanied to visits (for all outcomes), history of cognitive impairment (all but SWRT), ever used tetrabenazine (all but TFC), ever used antipsychotics (all but TMS) and age of onset (all but TFC and cUHDRS).

Though causality of the associations and collinearity between features should be further explored, the results suggest the important predictors that are candidates for statistical control in further analyses of observational data in HD.

This study was funded by F. Hoffmann-La Roche Ltd.

Lay Summary: Using data from Enroll-HD, we showed that beyond clinical measures, the product of an individual's CAG repeat length and age, CAG repeat length itself and Independence Scale score are the best predictors of Huntington's disease (HD) disease progression. Other factors such as attending visits accompanied, history of cognitive impairment and use of tetrabenazine or antipsychotics were also related to progression. These features should be taken into consideration when analyzing data in other HD studies.

32

Development of a Novel Patient-Reported Outcome Measure for Huntington Disease: The Huntington Disease Health Index (HDHI) Study

Alistair M. Glidden ${ }^{1}$, Elizabeth A. Luebbe ${ }^{1}$, Molly J. Elson ${ }^{2}$, Steven B. Goldenthal $^{3}$, Christopher W. Snyder ${ }^{4}$, Christine E. Zizzi ${ }^{1}$, Olivia S. Brumfield $^{1}$, Ray Dorsey ${ }^{1}$, Chad R. Heatwole ${ }^{1}$

${ }^{1}$ University of Rochester, Rochester, NY, USA; ${ }^{2}$ Emory University, Atlanta, GA, USA; ${ }^{3}$ University of Michigan, Ann Arbor, MI, USA; ${ }^{4}$ Rochester Institute of Technology, Rochester, NY, USA 
Background: Properly validated patient-reported outcome measures for use in Huntington disease (HD) clinical trials are needed for upcoming trials. Objective: To develop a multifaceted disease-specific patient-reported outcome measure for individuals with HD that can measure relevant changes in disease burden over time.

Methods: We performed 40 qualitative interviews and analyzed 2082 quotes regarding the symptomatic burden of HD. We subsequently performed a cross-sectional study with 389 participants to assess the prevalence and relative importance of 216 symptoms in HD. We selected questions for the Huntington Disease Health Index (HD-HI) to maximize its usability, responsiveness, and overall relevance to individuals with HD. Similar symptomatic questions were statistically grouped to form 13 individual HD-HI subscales. We performed cognitive interviews, test-retest reliability testing, and known groups validity testing on the HD-HI to optimize and further validate the instrument.

Results: Beta testing was conducted with 15 participants, as was testretest reliability testing. The wording of one question was modified based on participant input. Test-retest reliability demonstrated the reliability of the instrument while known groups testing demonstrated the capability of the instrument and its subscales to differentiate between groups of individuals with different levels of disease severity.

Conclusions: Initial evaluation of the HD-HI provides evidence that it is a valid and reliable outcome measure for serially assessing the multifaceted disease burden experienced by individuals with HD.

Sponsorship: Research support provided by NJ Cure and the Huntington Study Group.

Lay Summary: The Huntington Disease Health Index (HD-HI) is a valid, multifaceted, disease-specific patient-reported outcome measure designed to measure therapeutic gain during clinical trials. Here we describe its development and validation process in individuals with HD while outlining its key performance metrics.

\section{3}

Assessment of Mental Health Difficulties in Huntington's Disease: Does Informant Presence Make a Difference?

Sarah Gunn ${ }^{1}$, John Maltby ${ }^{1}$, Maria Dale ${ }^{2}$

${ }^{1}$ Neuroscience, Psychology and Behaviour, College of Life Sciences, University of Leicester, Leicester, LE1 9HN, United Kingdom; ${ }^{2}$ Leicestershire Partnership NHS Trust, Adult Mental Health Psychology, OSL House, East Link, Meridian Business Park, Leicester LE19 1XU, United Kingdom

Background: People with neurodegenerative conditions often have communication difficulties and cognitive impairments, making mental health assessment difficult. Informants close to the patient are often included in assessment, but effects of informant presence are unevaluated. This study investigated effects of informant presence during assessment of people with Huntington's disease (HD).

Methods: 4109 people split into four subsamples (manifest HD, premanifest carriers, genotype-negative and relatives not at genetic risk) were compared on the short-form Problem Behaviors Assessment affect, apathy, and irritability subscales, comparing participant-only versus informant-present report. Differences in affect, apathy and irritability scores between participant-only and informer-present conditions were examined via multiple regression, controlling for demographic, diseaserelated and individual confounds.

Results: Significant differences in apathy and irritability were found between participant-only and informant-present conditions for the premanifest, manifest and genotype-negative subsamples. When controlling for confounds, informant presence still significantly increased reported irritability for the manifest, premanifest and genotype-negative groups, and significantly increased apathy for the manifest group.

Discussion: Apathy may be systemically underreported in participantonly interviews, supporting prior findings of people with HD underreporting mental health symptoms. Affect was uninfluenced by informant presence, potentially due to lower symptom observability. Irritability ratings were higher with informant present for both HD and non-HD individuals, suggesting that underreporting via self-report may be attributable to non-HD factors.

Implications: Informant contributions to apathy assessments may be particularly important for people with HD. Clinicians should also note potential underreporting of irritability and affect, which is not remediated by incorporating informants.

Lay Summary: Mental health difficulties in people with $\mathrm{HD}$ are common, but often not recognized/expressed by the individual. Clinicians therefore rely on relative/carer input to identify such difficulties. We found this input is crucial for accurately assessing irritability/apathy, but low mood may still be underreported; this has important implications for assessment and support.

\section{4}

Development of Assessments for Later Stage Huntington's Disease: UHDRS Structured Interview of Function and HD Clinical Status Questionnaire

O.J. Handley ${ }^{1}$, S. Capodarca ${ }^{2}$, S. Frank ${ }^{3}$, R.L.M. Fuller ${ }^{4}$, M. Orth ${ }^{5}$, J.L. Levey $^{4}$, E. Neacy ${ }^{4}$, C. Sampaio ${ }^{4}$

${ }^{1}$ Cardiff University, UK. European Huntington's Disease Network; ${ }^{2}$ European Huntington's Disease Network; ${ }^{3}$ Beth Israel Deaconess Medical Center, USA; ${ }^{4}$ CHDI Management, Inc.; ${ }^{5}$ Ulm University, Germany. European Huntington's Disease Network

Background: The purpose of this study is to develop two assessments that can be used to measure critical milestones and events during the later stages of Huntington's disease (HD). An important aspect of the evaluation will be to assess whether the assessments can be administered to a companion either in-person or remotely (i.e. by phone contact with the companion). Therefore, these assessments will be evaluated for their internal consistency, reliability and validity.

Objectives: To evaluate these assessments for their internal consistency, reliability and validity.

Methods: Up to 170 dyads of Manifest HD Gene Expansion Carrier Participants and their Companion Participants are planned to be enrolled in this study from approximately 15-20 English-speaking study sites. The study includes two sequential parts, Part 1 and Part 2. Part 1 of this study will evaluate the internal consistency, reliability and validity of the Unified Huntington's Disease Rating Scale Structured Interview of Function (UHDRS SIF) using a cross-over design of the original UHDRS Total Functional Capacity, Functional Assessment Scale and Independence Scale (UHDRS TFC, FAS and IS) and the UHDRS SIF. The UHDRS SIF is a structured interview based on the UHDRS function scales (TFC, FAS and IS) and is administered to a Companion Participant only, either remotely or in-person. Part 2 will assess the clinimetric properties of the HD Clinical Status Questionnaire (HDCSQ), a questionnaire designed specifically to capture information on disease milestones that occur during the later stages of $\mathrm{HD}$, and the UHDRS SIF using a crosssectional design.

Current status and outlook: This study is in start-up. The First Participant In (FPI) is expected during 1Q2020. Study recruitment is scheduled to end during 2021. Preliminary results from Part 1 will be available during 2020 and full report will be available by the end of 2020 . Upon establishing the internal consistency, validity, and reliability these assessments may be incorporated into a large scale, global observational study of HD and/or other HD clinical studies as well as use them for planning clinical trials.

Lay Summary: The purpose of this study is to develop two assessments that can be used to measure critical milestones and events during the later stages of Huntington's disease. An important aspect of the evaluation will be to assess whether the assessments can be administered to a companion either in-person or remotely (i.e. by phone contact with the companion). 
35

Main Features of Mortality in Huntington's Disease: Analysis from Enroll-HD Dataset

Ilka Hatakeyama ${ }^{1,2}$, Paddy Byrne ${ }^{2}$, Jennifer Hoblyn ${ }^{1,2}$, Cristin Ryan ${ }^{2}$ ${ }^{1}$ Bloomfield Hospital, Dublin, Ireland; ${ }^{2}$ Trinity College Dublin, Dublin, Ireland

Background: Huntington's Disease (HD) is an autosomal dominant inherited disorder of a progressive nature. It involves a combination of motor, psychiatric and cognitive symptoms that ultimately leads to demise within 15-20 years from initial presentation. The advanced stages of HD require specialized multidisciplinary care in order to maximize the quality of life for these individuals and their family members. However, data describing the later stages of HD is scarce and published studies are limited. Aims and Objectives: The aim of this study is to depict the main features of mortality in HD and to identify any risk factors that may be influencing death rates. This will by achieved by analyzing data collected by EnrollHD, a fully integrated clinical research platform.

Methods: Data was obtained from Enroll-HD in a total of 15,301 participants. From this, the deaths of those individuals with manifest HD will be analyzed $(N=477)$. Statistical analysis will be performed with SPSS software (version 25). Descriptive statistic results for continuous variables will be presented as mean ( \pm standard deviation) whereas proportions will be applied for categorical variables. Some of the variables such as age, onset of symptoms, other comorbidities and habits will also be tested as covariates. Results: As a primary outcome, it is intended to have a clear description of the elements involved in the mortality of HD patients. We will identify and describe any correlations between variables and mortality, in order to determine potential risk factors for death in HD.

Lay Summary: In order to develop specialized multidisciplinary care and maximize the quality of life for HD patients, up-to-date data and indepth research about the late stages of the disease and description of main aspects of death are essential. The aim is to depict mortality and to identify risk factors that may be influencing death rates.

\section{6}

Experiences of Unauthorized Genetic Testing Disclosure Among Young People

Bonnie L. Hennig-Trestman, Catherine Martin

Huntington's Disease Youth Organization, Boca Raton, FL, USA

Background: Although predictive testing (PT) is accessible, the percentage of people at-risk who request PT is estimated to range between 3 and $25 \%$. Factors such as psychological and social challenges are suggested to contribute to such low utilization of PT. This study seeks to examine the personal experiences of at-risk individuals that in fact contribute to such low PT utilization.

Methods: Through social media platforms such as Facebook and Instagram the HD Youth Organization (HDYO) initially asked young people $(<35$ yo) affiliated with HDYO to share their testing experiences. Due to the frequency of respondents reporting experiences of unauthorized disclosure to test or disclosure of results for themselves or family members. HDYO then created a one-question online survey asking, "Have you ever experienced a time where a family member's HD information (or even your own information) was told to someone without permission?"

Results: Fifty-one young people responded to this inquiry. Twenty people $(40 \%)$ affirmed that a family member's or their own information was disclosed without their consent. Seven of the 20 respondents added comments. Each comment described incidences where healthcare practitioners disclosed one or more elements of HD testing information: healthcare practitioners included parent's HD status in the respondent's medical record; calls to family members answering questions about testing when the young person specifically indicated not to contact family members; a healthcare professional asked about CAG repeats for another family member even though the young person didn't know the family member was tested; and a situation where the healthcare provider commented that the respondent was the "last of the family to get tested" even though the person was unaware of others family members' testing status.

Conclusion: Although best practices have been developed to protect people at-risk, it remains important to educate healthcare providers on how to assure unintentional disclosure is avoided.

Lay Summary: Forty percent of young people who responded to a social media inquiry about unauthorized discloser during genetic testing affirmed that a family member's or their own information was disclosed without their consent. Although best practices have been developed to protect people at-risk, it remains important to educate healthcare providers on how to assure unintentional disclosure is avoided.

\section{7}

Analyzing Sex Differences in Huntington's Disease Using Enroll HD

Samantha Hentosh ${ }^{1}$, Weldon Furr ${ }^{2}$, Liang Zhu ${ }^{1}$, Natalia P. Rocha ${ }^{1}$, Erin Furr Stimming ${ }^{1}$

${ }^{1}$ UTHealth McGovern Medical School, Houston, TX, USA; ${ }^{2}$ LSU Health Shreveport, Shreveport, LA, USA

Rationale: Neurodegenerative diseases are influenced by a multitude of factors from genetics to environmental exposures. While much is known about the genetics of Huntington's disease (HD), sex-dependent differences in outcomes for this particular disease have not been fully uncovered. Other neurodegenerative diseases have been shown to have clear sex-dependent differences in disease prevalence and onset. Interpretation of these differences has an important role in optimizing treatment and better understanding disease mechanism in HD.

Objective: This study was designed to analyze sex-dependent differences in manifest HD patients from the Enroll HD database.

Methods: Longitudinal study including data from baseline and follow-up visits of manifest HD patients $(N=8043)$ from the Enroll-HD database (PDS4). Linear mixed models (GLIMMIX procedure) were used to assess sex-dependent differences regarding the motor, behavioral, and cognitive functioning over a series of 4 annual visits.

Results: Manifest HD patients showed significant sex-dependent differences in motor and depressive symptoms. Both sexes showed worsening motor and depressive symptoms over the course of 4 annual visits, female patients consistently presented with more severe motor and depressive symptoms than males $(p<0.0001)$. We did not find any difference between sexes regarding age at clinical $\mathrm{HD}$ diagnosis or the number of $\mathrm{CAG}$ repeats. There were no sex-dependent differences when we evaluated cognitive performance and severity of apathy, irritability/aggressive, psychosis and executive function scores as measured by the Problem Behaviors Assessment - Short (PBA-s) over the four visits.

Conclusions: Our analyses suggest that females overall have worse motor and depressive symptoms than males in the course of HD progression. The importance of continuing to identify sex differences in disease phenotype, progression, and neuropathology is critical for further advancing our understanding and treatment of HD.

Lay Summary: This study analyzes sex-dependent differences in Huntington's disease using a database that collects information of those affected (Enroll HD). It is important to the HD community because knowledge of sex-dependent differences may inform advancement in treatment and better understanding of disease mechanism.

\section{8}

First FDA-approved Human AAV Gene Therapy Clinical Trial in Adults with Early Manifest Huntington's Disease 
Ralf Reilmann ${ }^{1}$, Christopher Ross ${ }^{2}$, Paul S. larson $^{4}$, Michael D. Geschwind $^{5}$, Russell R. Lonser ${ }^{6}$, William S. Anderson ${ }^{3}$, Claudia M. Testa $^{8}$, Samuel A. Frank ${ }^{9}$, Sandra K. Kostyk ${ }^{7}$, Jee Bang ${ }^{2}$, Melvin Evers ${ }^{10}$, Martin de Haan ${ }^{10}$, Astrid Valles-Sanchez ${ }^{10}$, Kaity Renaud ${ }^{11}$, Lori Enney ${ }^{11}$, Patrick $\mathrm{Yu}^{12}$, Alex Kuta ${ }^{12}$, Scott McMilllan ${ }^{13}$, Jacek Lubelski ${ }^{13}$, Jaap Twisk $^{13}$, Pavlina Konstantinova ${ }^{10}$, Sander van Deventer ${ }^{10}$, and Joseph J. Higgins ${ }^{14}$ on behalf of the AMT-130 study team*

${ }^{1}$ George Huntington Institute, Muenster, Germany; Johns Hopkins School of Medicine, ${ }^{2}$ Department of Neurology, ${ }^{3}$ Department of Neurosurgery, Baltimore, MD USA; University of California San Francisco, ${ }^{4}$ Department of Neurological Surgery, ${ }^{5}$ Department of Neurology, San Francisco, CA USA; Ohio State University, ${ }^{6}$ Department of Neurosurgery, ${ }^{7}$ Department of Neurology, Columbus, $\mathrm{OH}$ USA; ${ }^{8}$ Virginia Commonwealth University, Department of Neurology, Richmond, VA USA; ${ }^{9}$ Harvard Medical School, Beth Israel Deaconess Medical Center, Department of Neurology, Boston, MA USA; uniQure BV, ${ }^{10}$ Research and Development, Amsterdam, The Netherlands, ${ }^{11}$ Clinical Operations, ${ }^{12}$ Regulatory, ${ }^{13}$ Chemistry, Manufacturing and Controls, and ${ }^{14} \mathrm{Clinical}$ Development, Lexington, MA USA

Objective: Design a Phase I/II clinical trial to explore the safety, tolerability, and efficacy of striatally-administered recombinant adenoassociated virus serotype 5, microRNA huntingtin (rAAV5-miHTT; AMT-130) gene therapy to lower huntingtin protein (HTT) in the brains of adult, early-manifest Huntington's disease (HD) patients.

Background: AMT-130 uses a therapeutic approach that efficiently lowers HTT mRNA and HTT protein by an RNA interference mechanism that targets the toxic mutant HTT (mHTT) exon 1 and avoids off-target effects. It is produced in large-scale commercial-grade quantities using a baculovirus expression system and insect cell line. Neuropathology, motor-function, and survival improve when mHTT is lowered by $25 \%$ to $75 \%$ in the brains of transgenic HD animal models. Studies in small and large animals performed under GLP regulations show that high doses of AMT130 are safe and well-tolerated after intra-striatal administration of AMT130 by convection enhanced delivery.

Design/Methods: The human equivalent dose (HED) to lower mHTT was extrapolated using a regression plot based on the brain biodistribution in small and large HD transgenic models. A clinical trial was designed using preclinical data and historical patient data from the TRACK-HD study.

Results: Bioanalytical modeling indicated that the AMT-130 HED to achieve a $75 \%$ lowering of mHTT in the striatum and $50 \%$ in the frontal cortex was $6 \times 1013$ AMT-130 genome copies/brain administered in a single dose. An analysis of historical HD patient data suggested that an 18-month, double-blind, imitation-surgery (sham)-controlled clinical trial enriched for early manifest subjects with $\geq 44$ HTT CAG repeats, was optimal to evaluate primary outcomes of safety and tolerability, and exploratory efficacy signals in mHTT, NF-L, imaging, motor, and functional measures.

Conclusion: We designed a clinical trial to assess safety and explore changes in clinical parameters and biomarkers related to HD progression. This clinical trial was approved by the FDA to enroll HD subjects in 2019. Lay Summary: We report the design of the first FDA-approved AAV gene therapy trial in adult patients with early manifest HD ( $\geq 44$ HTT CAG repeats) that will enroll patients in 2019. AMT-130 is a one time therapy, delivered intrastriatally, and uses a novel RNA interference approach that targets both full length HTT protein aggregates and importantly, also the highly toxic HTT exon1 protein fragments.

\section{9}

Clinical Studies of Deep Brain Stimulation for Huntington Disease with Chorea

AnChao Yang ${ }^{1}$, KaiLiang Wang ${ }^{1}$, Yue Huang ${ }^{2,3 \#}$, Jean Marc Burgunder ${ }^{4,5,6}$, HongQiu Gu ${ }^{2}$, Kai Zhang ${ }^{1}$, HuanGuang Liu ${ }^{1}$, Zhong $\mathrm{Pei}^{5}$, HuiFang Shang ${ }^{6^{*}}$, FanChao Meng ${ }^{1}$, JianGuo Zhang ${ }^{1 *}$
${ }^{1}$ Functional Neurosurgery Department, Beijing Tiantan Hospital, Capital Medical University, China; ${ }^{2}$ China National Clinical Research Centre for Neurological Diseases, Neurology Department, Beijing Tiantan Hospital, Capital Medical University, China; ${ }^{3}$ Neurology Department, University of Bern, Bern, Switzerland; ${ }^{4}$ SoMS, Faculty of Medicine, UNSW Sydney, Sydney, Australia; ${ }^{5}$ Neurology Department, Huaxi Hospital, Sichuan University, Chengdu, China; ${ }^{6}$ Neurology Department, the 1st Hospital, Zhongshan University, Guangzhou, China; * corresponding authors, email: zjguo73@126.com; hfshang2002@163.com; " presenting author email: yue.huang@unsw.edu.au

Chorea in HD is difficult to manage clinically. Case reports and small series have suggested that deep brain stimulation targeting bilateral global pallidus internus (Gpi-BDS) may improve HD chorea. In our Functional Neurosurgery Department, Beijing Tiantan Hospital, we have performed Gpi-BDS on thirteen HD patients with medically refractory chorea from 2012 January to 2018 January. We followed them up for 6 months $(n=$ 13), 12 months $(n=11), 24$ months $(n=9)$, and 36 months $(n=4)$ and assessed them using motor section of the UHDRS. Chorea and dystonia subscores were significantly improved and maintained for up to 36 months. Total motor scores and hypokynesia sub-score were significantly improved up to 24 and 12 months respectively. Other subscores remained unchanged at any follow-up time-point. At 6 months follow-up, independence scale (IS), functional assessment (FA) and total functional capacity (TFC) were significantly improved. The data from our $13 \mathrm{HD}$ patients provide further evidence for a positive effect of Gpi-BDS in HD patients with Chorea. Therefore, we have set up to conduct a multicentred, blinded, prospective study in China to consolidate the findings. Patients will be recruited and followed-up at three centres (Beijing, Chengdu and Guangzhou). DBS will be conducted by the same neurosurgery team (Beijing Tiantan Hospital) to ensure the consistent quality. Patients will be randomly selected to stimulation ON or OFF right after operation. The primary aim of this study is to evaluate efficacy and safety of GPi-DBS in the treatment of chorea in patients with HD. Secondary aims include impact of GPi-DBS on quality of life, on cognitive, behaviour and functional performances of HD. Furthermore, relevant indicators of GPi-DBS during intraoperative monitoring for predicting postoperative clinical outcomes will be explored.

Lay Summary: Chorea in HD is difficult to manage clinically. From 2012.01 to 2018.01 , we have performed deep brain stimulation targeting bilateral global pallidus internus (Gpi-BDS) on 13 HD patients with significant improvement of HD chorea up to 36 months. Therein, we are about to conduct a multi-centred study to further consolidate the findings.

\section{0}

Anosognosia Related to Memory but not Executive Functioning, Motor Dysfunction, or Genetic Susceptibility in Huntington's Disease

Shelby B. Hughes, Ciaran M. Considine, Daniel O. Claassen Vanderbilt University Medical Center, Nashville, TN, USA (all authors)

Objective: Individuals with Huntington's disease (HD) frequently exhibit a lack of awareness for obvious disease manifestations, termed anosognosia. Existent research is limited and tends to focus on motor impairment (rather than neurobehavioral and functional manifestations). Neurobiological origins have been proposed (e.g., nondominant frontostriatal dysfunction), with cognitive models proposing mediation via memory and executive functioning impairment. This study measured anosognosia across multiple domains of symptoms in HD patients, then related severity of anosognosia to clinical indicators.

Method: 32 HD patients were administered Deckel \& Morrison's Anosognosia Scale (AS; 1996) as a component of routine 
neuropsychological evaluation (including assessment of memory and executive functions) within the HDSA COE clinic at the Vanderbilt University Medical Center. Domains rated included gait, manual dexterity, speech, cognition, and emotion-regulation. The AS calculates a severity rating based on the difference between patient and clinician's scores. Patient and clinician were blind to the other's ratings. Positive scores indicated overestimation of symptoms, negative scores indicated anosognosia. Genetic susceptibility (CAG repeats), physical symptom severity (UHDRS score), and demographic factors were collected.

Results: Descriptive analyses suggested a bi-modal presence/absence of anosognosia, without an overestimation group. Bivariate correlation noted a significant positive correlation $(\mathrm{r}=0.36, p<0.045)$ between AS score and Recognition on a memory measure (HVLT-R). Executive functioning performance, UHDRS score, and CAG repeats were not correlated with AS score.

Conclusion: Anosognosia may be mediated by encoding/registration memory dysfunction rather than executive dysfunction (e.g., working memory impairment). Anosognosia may arise in HD patients independent of pathological/motor-clinical severity.

Lay Summary: This study looks at potential contributory factors associated with anosognosia in the HD population, specifically cognitive functions and disease severity. Our study shows that anosognosia may be mediated by memory dysfunction, but not by executive functions or disease severity.

\section{1}

Comorbidities and Medications in Huntington's Disease, Parkinson's Disease and the General Population in a US Claims Database

Lianna Ishihara ${ }^{1}$, Edward Wild ${ }^{2}$, David Oliveri ${ }^{3}$

${ }^{1}$ Roche Products Ltd., Welwyn Garden City, UK; ${ }^{2}$ Huntington's Disease Research Centre, UCL Institute of Neurology, London, UK; ${ }^{3}$ Genesis Research, Hoboken NJ, USA

Truven MarketScan has been previously used to explore healthcare utilization and direct medical costs among Huntington's disease (HD) patients by stages of disease progression. This study characterizes the comorbidities, symptoms and treatment patterns of patients with HD compared with patients with Parkinson's disease (PD) and the general population (GP) excluding neurodegenerative diseases. US claims data can offer valuable insights on the natural history of HD and provide information on standard practices used in a real-world setting.

Patients were matched by age, gender, database type (Commercial/ Medicaid) and enrollment prior to the index date (1st January 2017). Diseases and medications selected for this study were related to HD and its symptoms, as well as psychiatric comorbidities, common diseases and commonly used medications. All comorbidities were reported by International Classification of Diseases Version 10 (ICD- 10) sub-heading, and specific comorbidities and symptoms defined using ICD-10 codes. Medications were reported by therapeutic class and defined using national drug codes.

A total of 587 patients with HD were matched to patients diagnosed with PD and GP controls, of which $453(77 \%)$ had at least one HD ICD-10 code recorded in 2017. Major depressive disorders and other anxiety disorders were the most frequently reported disease subheadings in patients with HD; incidences were also higher in patients with PD than in GP. Patients with HD used antidepressants and antipsychotic therapies most and at a higher frequency than in patients with PD and GP.

These results indicate that major depressive and anxiety disorders are common to both HD and PD relative to the GP. Antidepressants and antipsychotics are commonly used in HD. Further studies exploring specific comorbidities and their relationship to medication use in these patient populations are ongoing.
Funding Information: This study was funded by F. Hoffmann-La Roche Ltd.

Lay Summary:This study looked at the most common medical conditions that affect people living with Huntington's disease (HD). It also examined the type of drugs prescribed to patients to treat these conditions. The results were compared with patients living with Parkinson's disease (PD) and the general population. Anxiety and depression were more common in HD and PD than in the general population. Patients with HD were prescribed antidepressants and antipsychotic drugs more often than patients with $\mathrm{PD}$ and the general population.

\section{2}

A Fully Automatic Pipeline for Estimation of Putamen and Caudate Volume

Richard Joules ${ }^{1}$, Richard Parker ${ }^{1}$, Robin Wolz ${ }^{1,2}$

${ }^{1}$ IXICO Plc, London, UK; ${ }^{2}$ Imperial College London, London, UK

Introduction: The striatum is a prominent area in Huntington's pathology where longitudinal atrophy has been employed to assess disease progression and potential treatment effects in clinical trials. Common methods for assessing atrophy require cross sectional segmentations for initialisation. Additionally, cross-sectional volumes of striatal structures are required to estimate dosage in clinical trial interventions where compounds are directly administered to the striatum.

Several methods exist to segment the caudate and putamen, the commonly accepted gold-standard being manual segmentation. This is resource intensive and susceptible to inter- and intra-rater variability. Here, we present a method for fully automated segmentation of the caudate and putamen, LEAP (Wolz;Neuroimage;2010), as applied to a HD cohort.

Method: We have optimized a multi-atlas pipeline to generate segmentations of the caudate and putamen from T1W images in a HD cohort. In brief, images are brain extracted (Pincram;Heckermann;PLOS ONE; 2015) and inhomogeneity corrected (Avants;Neuroinfomratics;2011). Pre-processed inputs are compared to a reference database of $>500 \mathrm{im}-$ ages with pre-existing caudate and putamen masks. A consensus atlas is generated from the 10 most similar images which is subsequently refined to generate a final regional segmentation.

Results: Test sets of $100 \mathrm{~T} 1 \mathrm{~W}$ images, with manual caudate and semiautomated putamen "ground-truth" segmentations were processed with the LEAP pipeline. Comparison of LEAP results to the ground truth reveal significant correlations in estimated volumes, $r=0.96(p<<0.001)$, for both the caudate and putamen. A significant spatial overlap in segmentations, as measured with DICE was observed for both putamen and caudate with a mean dice DICE of 0.901 (sd:0.03) and 0.8 (sd:0.06) respectively.

Conclusion: We have presented a fully-automatic pipeline for caudate and putamen segmentation allowing for efficiency saving in clinical trial deployments and reduction of potential inter-segmenter variance when compared to manual segmentation.

Lay Summary: This work describes a fully automatic method for segmentation of the caudate and putamen, providing an efficient and robust method for cross-sectional volume estimates and defining regions of interests for estimating longitudinal atrophy in the striatum, a key biomarker in clinical trials of HD.

\section{3}

Early Motor Phenotype Influences Neuropsychiatric and Cognitive Disturbances in Huntington's Disease

Parunyou Julayanont ${ }^{1}$, Kenneth M. Heilman ${ }^{1,2}$, Nikolaus R. McFarland ${ }^{1}$ ${ }^{1}$ University of Florida College of Medicine, Gainesville, FL, USA; ${ }^{2}$ Malcom Randall Veterans Affairs Medical Center, Gainesville, FL, USA 
Objective: To determine the influence of motor phenotype on the characteristics of neuropsychiatric disturbances and cognitive impairments in the early motor-manifest Huntington's disease (HD) individuals.

Methods: From the ENROLL-HD study, 3505 HD individuals with $\leq 5$ years of motor duration were classified into 1125 chorea-dominant (C-HD), 867 Parkinsonism-dominant (P-HD) and 1513 mixed-motor (MHD) phenotype based on the Parkinsonism/chorea index. The short Problem Behaviors Assessment (assessing depression, irritability/aggressive, apathy, obsessive-compulsive/perseverative behaviors, and psychosis) and neuropsychological tests (Stroop Interference test, trail A and B, letter fluency, Symbol Digit Modality test and animal fluency) were analyzed.

Results: Motor phenotype influenced the neuropsychiatric and cognitive characteristics in early motor-manifest HD individuals. Independent to the age of onset, motor duration and severity of motor symptoms, the P-HD group had significantly higher severity of all neuropsychiatric clusters and lower performance on all cognitive tests than the M-HD and CHD group (all $p<0.001$ ). The C-HD group had less apathy and depression than the M-HD group. The P-HD group also had poorer total functional capacity than the C-HD and M-HD group $(\mathrm{p}<0.001)$.

Conclusions: In the early stage of motor manifestation, the Parkinsonism-dominant phenotype had more severity of various neuropsychiatric disturbances and cognitive impairments than the choreadominant and mixed-motor phenotype. A longitudinal study should explore the progression of neuropsychiatric, motor, and cognitive symptoms among these motor phenotypes.

Lay Summary: This is the first and largest study to show that early motor phenotype influences the neuropsychiatric and cognitive profiles in HD patients. The Parkinsonism-dominant phenotype had more severity of various neuropsychiatric disturbances and cognitive impairments than the chorea-dominant and mixed-motor phenotype. This will help clinicians in stratifying the HD patients with higher risk of behavioral and cognitive impairments based on the prominent motor symptoms.

\section{4}

Mild Cognitive Impairment and Dementia in Motor Manifest Huntington's Disease: Classification and Prevalence

Parunyou Julayanont ${ }^{1}$, Nikolaus R. McFarland ${ }^{1}$, Kenneth M. Heilman ${ }^{1,2}$ ${ }^{1}$ University of Florida College of Medicine, Gainesville, FL, USA; ${ }^{2}$ Malcom Randall Veterans Affairs Medical Center, Gainesville, FL, USA

Objectives: To identify the characteristics and prevalence of mild cognitive impairment in patients with motor-manifest Huntington's disease (HD) and to propose a new mild cognitive impairment (HD-MCI) classification for $\mathrm{HD}$. Methods: From the ENROLL-HD, 307 motor-manifest HD patients who completed cognitive evaluations were included. Cognitive impairment in each domain was determined by the age- and education-adjusted cutoffs ( $>1.5$ standard deviations below the mean). HD-MCI was defined as an impairment in at least one cognitive domain without a loss of independence (Function Independence Scale, FIS $\geq 85$ ). Dementia (HD-Dem) was defined as at least two domains of cognitive impairment with functional impairment (FIS $\leq 80$ ).

Results: At the onset of motor manifestation, MCI was present in $84 \%$ and dementia in $5 \%$ of patients. After 5 years of motor abnormalities, $24 \%$ of participants met the criteria for MCI and $69 \%$ for dementia. Executive dysfunction including slow processing speed were the most prevalent impaired domains found in $70 \%$. Language impairment was reported in $55 \%$ and memory deficits were reported in 53\%. To classify subtypes of MCI, isolated impairments of "Executive Functions" (including processing speed) and "Representational Functions" (language and memory) were found in $30 \%$ and $15 \%$, respectively, while the combined impairments were reported in 55\% of HD-MCI cases.
Conclusion: A high prevalence of $\mathrm{MCI}$ is present even in the early stage of motor manifestation in HD. Based on the pathophysiology and prevalence of cognitive impairments; we classified MCI among HD patients into "Executive" MCI, "Representational" MCI and Combined "Executive- Representational" MCI.

Lay Summary: This study is the first report on the prevalence of cognitive impairment (MCI and dementia) and proposes a classification of MCI subtypes (Executive" MCI, "Representational" MCI and Combined "ExecutiveRepresentational" $\mathrm{MCI}$ ) among motor-HD individuals. It shows that, even in the early phase of motor manifestation, the prevalence of MCI is high in these patients and this should warrant more attention from health care providers.

\section{5}

Fall Risk and Functional Balance Are Related to Postural Sway in Huntington's Disease

Deb Kegelmeyer ${ }^{1}$, Sandra Kostyk ${ }^{2}$, Doris Walterhouse ${ }^{1}$, Anne Kloos ${ }^{1}$ ${ }^{1}$ School of Health and Rehabilitation Sciences, The Ohio State University, Columbus, OH, USA; ${ }^{2}$ Department of Neurology, The Ohio State University, Columbus, OH, USA

Background: Postural instability is a common problem in Huntington's disease (HD), yet little is known about trunk control and postural stability as it relates to functional mobility and falls. This study compared postural sway between individuals with $\mathrm{HD}$ and controls in relation to functional gait, balance, and falls measures.

Methods: Postural sway was measured under 4 conditions (standing on firm and foam surfaces; eyes open and closed) using the Boditrak2 pressure-sensing mat. Sway measures assessed: 1) maximal center of pressure distance reached $\left.\left(\mathrm{COP}_{\max }\right), 2\right)$ total $\mathrm{COP}$ distance traveled $\left.\left(\mathrm{COP}_{\text {total }}\right), 3\right)$ right and left weight distribution (percent). In addition, gait and balance function were assessed using the $25 \mathrm{ft}$ walk test, the Functional Gait Assessment (FGA), Tinetti mobility test (TMT), and six month fall history.

Results: Nineteen individuals with HD (mean age 53 y.o.; 7 males; mean years since onset 7.5); and 11 controls (mean age 43 y.o.; 6 males) participated. Postural sway differed between those with HD (59.7") and controls $\left(43.4^{\prime \prime}\right)$ only in $\mathrm{COP}_{\text {total }}$ on a firm surface with eyes open $(p=.038)$. Six individuals with $\mathrm{HD}$ were unable to stand on foam with eyes closed for the full $30 \mathrm{~s}$ while all controls completed this condition $(p=.038)$. In individuals with $\mathrm{HD}$, fallers $(n=10)$ exhibited significantly unequal left and right weight shift and $\mathrm{COP}_{\max }$ when standing on foam in both the eyes open and closed conditions $(p<.05)$ than non-fallers. Lower TMT and FGA scores correlated with higher $\mathrm{COP}_{\text {total }}$ on firm surfaces, eyes closed $(\mathrm{r}=-.496$ and $-.575 ; \mathrm{p}$ $<.05$ ). Gait speed did not correlate with postural sway.

Conclusions: Postural sway measures while standing on dense foam may be a simple useful tool for fall risk screening in HD. Greater multidirectional sway standing on a firm surface with eyes closed may indicate balance deficits that affect gait and fall risk.

Lay Summary: To prevent falls we must find the underlying cause so that we can develop better treatments. This study explored a simple tool for measuring trunk sway and examined the relationship between sway and falls. There was a relationship between history of falling and increased sway standing on a soft surface.

\section{6}

\section{HD Patients Facing Challenges in Pakistan}

Asif Khan

Huntington Disease Care and Cure Society of Pakistan

So far in Pakistan, no statistical data has been collected. Huntington Disease and other related degenerative disorders, although considered a rare entity, are now on the increase in our country. 
Although structured survey and prevalence to determine the incidence have not been done in Pakistan, it is postulated from certain indirect studies that our 15,000 family members may require support and information in the KPK region about this disease. In Pakistan people are reluctant to disclose their disease because of culture, so it is also worth mentioning that the number of affected people may be much higher.

There is no "Specialized Treatment Center" for degenerative neurological disorders anywhere in Pakistan. These disorders are dealt with by general physicians or internists, but they only manage the medical side of the treatment, about $20 \%$ of a patient's need, whereas the remaining $80 \%$ of the management of these disorders is missing. Therefore, in the care and treatment of such patients, the larger element has completely been neglected or has not been taken care of. This leaves these patients at mercy of the drugs only, which have a limited role in the treatment of such disorders as proven by many studies and reviews of randomized control trials conducted in the West.

Therefore, there is a dire need for an institute where the medical treatment as well as general supportive care and rehabilitation of these patients can be carried out.

\section{Patient Services: Must Meet Urgent Needs Now.}

Accordingly, the Huntington Disease Care and Cure Society of Pakistan assists people affected by this devastating nerve disorder, both directly and indirectly, to boldly face the painful and miserable death it brings. This is possible through unconditional quality care, treatment, counseling, and support of the people. The society's foremost objective is to establish a nursing home at Peshawar, and then a chain of network-clinics and nursing homes in the capital cities of the other three provinces in the long run, provided the resources permit.

Education, Awareness, Understanding and Know-How.

To ensure this noble cause is successful, the society is bent on educating the lay public by disseminating information by organizing awareness programs and campaigns on electronic and print media through bulletins, journals, and audio-video-conferences.

Lay Summary: Huntington Disease Care and Cure Society of Pakistan is a curtain raiser against $\mathrm{HD}$, is committed to fight for humanity, under the patronage of I H A based in The Netherlands, to be up to the task and challenges in the future in the services of humanity. The society aspires to the following goals and aims: 1) establishment of a nursing care home, 2) establishment of neuro rehab centers, 3 ) creating a center of excellence for educational programmes, and 4) establishing online telephone services $24 \mathrm{~h}$ for counseling, education, advocacy, and support to family and HD patients.

47

\section{Characteristics of Patients on First Visit to an HD Clinic}

Jennifer A Klapper, Tanya Bardakjian, Pedro Gonzalez-Alegre

HDSA Center of Excellence, Pennsylvania Hospital, Department of Neurology, University of Pennsylvania, Philadelphia, PA, USA (all authors)

Background: There are two main types of new patient visits (NPV) in an HD center, patients diagnosed with HD and subjects interested in predictive testing (PT). Understanding their needs is necessary to offer appropriate services.

Objective: To describe the demographic and clinical characteristics of new patients presenting to an HD center for their first evaluation.

Methods: We retrospectively collected demographic and clinical data from NPV at our HD Clinic between January 2018 June 2019.

Results: There were $86 \mathrm{NPV}, 54$ PT and 32 with HD. PT patients were younger, had a higher BMI and, unsurprisingly, lower mUHDRS scores than those with HD. All new patients were seen by the neurologist and genetic counselor. Evaluation by the clinic's psychiatrist was required in $46 \%$ PT and $75 \%$ of HD patients, and social worker help was requested in $20 \%$ of PT and $56 \%$ of HD patients. Reporting of symptoms differed between both groups, with a predominance of cognitive and motor in HD patients and psychiatric in those that came for PT. In both groups, the most commonly prescribed medications targeted psychiatric symptoms. $41 \mathrm{PT}$ patients proceeded to testing, 7 declined, 2 required stabilization of psychiatric symptoms before testing and 3 were not offered testing at the NPV for different reasons. Of the 41 tested, 20 were gene negative, 18 positive and 3 had intermediate repeats (27-35). Correlation of psychiatric symptoms and BMI with the outcome of testing will be presented.

Conclusions: Our data indicate that, despite different utilization of services between HD patients and those seeking PT, a neurologist, psychiatrist, genetic counselor and social worker should be available for all NPV. A large number of PT patients exhibit psychiatric symptoms.

Lay Summary: This data provides additional illustrations pointing to the need for a multi-disciplinary HD clinic which provides neurology, psychiatry, social work and genetic counseling to patients and families presenting for their initial visit for HD-related health care concerns. The expert assessment of several HD specialists, to identify needs and an optimal plan of care, in the context of a complex illness such as HD, is crucial for individuals and families establishing a relationship for care in an HD Center.

\section{8}

CAG Length Does Predict Duration of Huntington's Disease Illness

Douglas R Langbehn

University of Iowa, Iowa City, Iowa, USA

Background: There are published claims that, although CAG length is a strong predictor of HD onset-age, it surprisingly does not predict the duration of clinical illness until the time of death. However, we have noted a potential, subtle source of confounding not addressed in the underlying statistical models. We therefore reanalyzed one of the underlying datasets with an alternative model that addresses this potential confounding.

Methods: For the purpose of this reanalysis, we were granted access by EHDN to the final Registry database. We fit Cox proportional hazard survival models for the time from HD onset until death and included both CAG length and age of HD onset as predictor variables. In alternative models, we defined onset as the age of UHDRS diagnosis or the clinical rater's estimate of symptom onset-age. We first fit simple linear models to test the null hypotheses of no age and no CAG effect. We followed this with nonlinear models that more accurately described the relationships between CAG length, onset-age, and time until death. We used agestratified Kaplan-Meier survival plots to illustrate the possible CAG effects in the raw data.

Results: After controlling for onset-age (using either definition), CAG length has a highly significant influence on the remaining lifespan. For subjects experiencing onset at the same age, prognosis is clearly worse for those with longer CAG lengths. The phenomenon is easily observed in age-stratified plots of the raw data. The proportional hazard influences of onset-age and CAG are both nonlinear and can be illustrated using restricted cubic spline models. Conclusion: Conditional on onset-age, CAG length does indeed influence length of HD illness until death. Previous analyses suggesting the contrary did not consider the influence of onset-age, which tends to be younger with longer CAG lengths. Perhaps agedependent protective factors partially mask this CAG influence? Lay Summary: It has recently been claimed that the length of the CAG expansion does not influence the remaining lifespan after the onset of HD illness. Reanalysis of one of the data sources used to 
claim this suggests that this is not the case. For people with similar ages of onset, longer CAG lengths are associated with shorter remaining lifespans.

\section{9}

\section{An Atlas of Age and CAG-Dependent HD Phenotypes}

Douglas R Langbenh ${ }^{1}$, Cristina Sampaio ${ }^{2}$, Clement $\mathrm{T}$ Loy $^{3}$, Amrita Mohan $^{2}$, Elizabeth McCusker ${ }^{4}$

${ }^{1}$ University of Iowa, Iowa City, Iowa, USA; ${ }^{2}$ CHDI Foundation, Princeton, New Jersey, USA; ${ }^{3}$ Sydney School of Public Health, University of Sydney, Sydney, NSW, Australia; ${ }^{4}$ Sydney University Medical School, University of Sydney, Sydney, NSW, Australia

Objective: To document the range of Huntington disease (HD) phenotypes in detail for various CAG repeat lengths, age-ranges, and declining functional levels.

Introduction: A fundamental feature of $\mathrm{HD}$ is the variable $\mathrm{CAG}$ repeat expansion in the Huntingtin gene. Much is known about the variation in onset of motor manifestations and its inverse relationship to expansion length. The wider phenotype - including non-motor features - at particular CAG lengths, ages, and functional levels is less well characterized. $\mathrm{Up}$ to $70 \%$ of motor onset variability is estimated to relate to the CAG expansion in the Huntingtin gene on chromosome 4. Beyond this, genetic and environmental modifiers may influence the disease phenotype. To date, limited but consistent progress has been made in the search for genetic and environmental modifiers. These have been identified by quantitative differences in disease progression. Less studied is the possible modifier impact on the qualitative HD phenotype.

We created the Enroll-HD Phenotype Atlas to summarize the range and distribution of HD phenotypes, including outliers and possible clusters, for the range of CAG lengths, ages, and functional levels.

Methods: The December 2018 Enroll-HD data-cut contains 27,554 observations of 11,429 individuals with HD gene CAG expansions. This large sample allows detailed observational summaries by five-year age range for all common HD CAG lengths. Further, it allows descriptive plots relating specific ENROLL measurements to age and CAG.

Results: This descriptive atlas is an initial data-reduction that allows easier comprehension of the Enroll-HD data. It is useful for understanding typical HD states and for judging the degree to which an individual's phenotype is atypical. The observable patterns in the atlas should also be a catalyst to more formal multi-domain characterization of motor, cognitive, and psychiatric progression and its relationship to functional decline and diseasemodifiers. This web-based atlas will soon be available via CHDI.

Lay Summary: The Enroll database now consists of over 27,000 observations from more than 10,000 people with the Huntington's Disease CAG expansion. We are providing an organized summary of the range and distribution of clinical research measures that are typical at various ages for all commonly encountered CAG lengths. This will assist investigators and clinicians in their understanding of typical and atypical features of HD and identification of individuals or groups with illness patterns that deserve additional treatment considerations and further research.

50

Preliminary Results from an Ongoing Open-label Extension (OLE) Study Investigating RG6042 Huntingtin Protein (HTT) Antisense Oligonucleotide (ASO) in Adults with Manifest Huntington's Disease (HD)

Blair R Leavitt ${ }^{1}$, Sarah J Tabrizi ${ }^{2}$, Patricia Sanwald Ducray ${ }^{3}$, Edward J Wild $^{2}$, Valerie

Schlegel $^{4}$, Gregory Hooper ${ }^{5}$, Alessia Nicotra ${ }^{5}$, Jestinah Chevure ${ }^{5}$, Anne Smith $^{6}$, Roger Lane ${ }^{6}$, Frank Bennett ${ }^{6}$, Lauren Boak ${ }^{4}$, Rachelle Doody ${ }^{4,7}$, and Scott Schobel ${ }^{4}$ on behalf of the OLE investigator network
${ }^{1}$ Centre for Molecular Medicine and Therapeutics, Department of Medical Genetics, University of British Columbia, Vancouver, BC, Canada; ${ }^{2}$ UCL Huntington's Disease Centre, UCL Queen Square Institute of Neurology, University College London, London, UK; ${ }^{3}$ Roche Pharma Research and Early Development, Roche Innovation Centre Basel, Basel, Switzerland; ${ }^{4}$ F. Hoffmann-La Roche Ltd., Basel, Switzerland; ${ }^{5}$ Roche Products Ltd., Welwyn Garden City, UK; ${ }^{6}$ Ionis Pharmaceuticals Inc., Carlsbad, CA, USA; ${ }^{7}$ Genentech Inc., South San Francisco, CA, USA

Long-term safety, tolerability, biomarker and clinical effects of the HTT-lowering ASO RG6042 are currently being evaluated in a global clinical programme, including an ongoing 15-month OLE study (NCT03342053) in 46 individuals who previously participated in the Phase I/IIa study (NCT02519036). Patients in the OLE were randomized to receive $120 \mathrm{mg}$ RG6042 either every 4 weeks (Q4W) or every 8 weeks (Q8W).

At the time of abstract submission, over 400 doses of RG6042 have been administered in participants of this study, with all individuals having received at least 11 doses $\mathrm{Q} 4 \mathrm{~W}$ or six doses $\mathrm{Q} 8 \mathrm{~W}$. The safety and tolerability data to date suggest that RG6042 is well tolerated over greater than one year of treatment. There were fewer overall adverse events (AEs), serious AEs and potentially drug-related complications in the Q8W regimen than the $\mathrm{Q} 4 \mathrm{~W}$ regimen.

Cerebrospinal fluid (CSF) mutant HTT (mHTT) data available at a 9-month analysis suggest that CSF mHTT levels can be lowered by a median of $47 \%$ in the Q8W regimen and $66 \%$ in the Q4W regimen with both magnitudes exceeding the minimum considered pharmacologically relevant. Transient increases in CSF neurofilament light chain and Tau are observed in both the Q4W and Q8W groups from data available to date which decreased on continued treatment. The plasma exposure (pharmacokinetics [PK]) and CSF mHTT reduction (pharmacodynamics [PD]) from this study informed a PK/PD model which has been used to simulate a less frequent dosing regimen (every 16 weeks) that would achieve pharmacologically relevant targets for potential efficacy - a hypothesis currently being tested in the Phase III GENERATION HD1 study.

Funding Information: The OLE study was funded by Ionis Pharmaceuticals Inc. and F. Hoffmann-La Roche Ltd.

Lay Summary: Lowering the huntingtin protein (HTT) is a promising therapeutic strategy in Huntington's disease (HD) which has the potential to slow or stop clinical disease progression. Long-term information about lowering HTT in people with HD using RG6042, an antisense oligonucleotide injected into the spinal fluid, is currently being collected in a global clinical programme. Results from the 15-month extension of the Phase I/IIa study on the safety, tolerability and biological response of RG6042 in patients with HD are being presented.

51

Intrathecal Drug Delivery of Antisense Oligonucleotides in Huntington's Disease: Experience of Ionis/Roche RG6042 Development Programme and Best Practice Considerations for Real-World Use

Blair R Leavitt ${ }^{1}$, Edward J Wild ${ }^{2}$, Jee Bang ${ }^{3}$, Anne Smith $^{4}$, Valerie Schlegel ${ }^{5}$, Scott Schobel ${ }^{5}$, Alessia Nicotra ${ }^{6}$, G. Bernhard Landwehrmeyer $^{7}$, Sarah J Tabrizi ${ }^{2}$, and Mark Guttman ${ }^{8}$ on behalf of the OLE investigator network

${ }^{1}$ Centre for Molecular Medicine and Therapeutics, Department of Medical Genetics, University of British Columbia, Vancouver, BC, Canada; ${ }^{2}$ UCL Huntington's Disease Centre, UCL Queen Square Institute of Neurology, University College London, London, UK; ${ }^{3}$ Department of Neurology, Johns Hopkins University School of Medicine, Baltimore, MD, USA; ${ }^{4}$ Ionis Pharmaceuticals Inc., Carlsbad, CA, USA; ${ }^{5}$ F. Hoffmann-La Roche Ltd., Basel, Switzerland; ${ }^{6}$ Roche 
Products Ltd., Welwyn Garden City, UK; ${ }^{7}$ University of Ulm, Dept of Neurology, Ulm, Germany; ${ }^{8}$ Centre for Movement Disorders, Toronto, ON, Canada

Intrathecal administration involves the introduction of a drug substance into the intrathecal (subarachnoid) space of the spinal column through a spinal needle inserted typically into the lower back via lumbar puncture (LP) or via a special device (e.g. a surgically implanted catheter connected to a drug reservoir). This procedure is used when drugs cannot cross the blood-brain barrier. Once injected, drugs administered via the intrathecal route are distributed within the cerebrospinal fluid from which they can access target tissues in spinal cord and brain. The method is commonly used in spinal anesthesia, pain management and chemotherapy; but with the advent of antisense oligonucleotides (ASO) for treatment of neurological conditions, the indications have expanded to include spinal muscular atrophy, and, as potential new indications, amyotrophic lateral sclerosis, Alzheimer's disease and Huntington's disease (HD).

We use the intrathecal route to deliver RG6042 (previously HTTRx), an investigational, huntingtin mRNA-targeting ASO to patients with HD. Considerable experience on intrathecal administration via LP has been gained in the RG6042 clinical development programme, including the double-blind, placebo-controlled, multiple ascending dose, Phase I/IIa study (NCT02519036) in 46 patients with early manifest HD and an ongoing Phase II open-label extension (NCT03342053) where patients receive $120 \mathrm{mg}$ RG6042 monthly or every other month in a 62-week treatment period (total procedures $\geq 400$ in the programme to date).

We will report on the experience with intrathecal drug delivery across these two studies and present information on suggested best practices in HD. Procedure-related videos will be shared, including use of spinal ultrasound. Finally, we will provide a forward-looking outlook on realworld considerations of implementing intrathecally delivered therapies in the clinical interface.

Funding Information: The studies were funded by Ionis Pharmaceuticals Inc. and F. Hoffmann-La Roche Ltd.

Lay Summary: Injection into the spine via lumbar puncture (known as intrathecal administration) can be used to deliver a drug to the brain when delivery by other methods (pills, intravenous administration, etc.) will not result in enough drug reaching the brain. Intrathecal administration is increasingly being used for drug delivery in neurological diseases. We have gained experience with intrathecal delivery during the clinical development programme of a new experimental drug, RG6042, for people with Huntington's disease (HD). The presentation will share lessons learnt so far and present information about this important procedure that allows antisense oligonucleotide huntingtin-lowering drugs to be given to people with $\mathrm{HD}$.

\section{2}

Utilizing 24(S)-hydroxycholesterol to Identify Patient Populations and Clinical Endpoints: Negative Emotion Processing in Huntington's Disease as Proof of Principle

Michael Lewis ${ }^{1}$, Jing Dai ${ }^{1}$, Joe Kennedy ${ }^{1}$, Beth Borowski ${ }^{2}$, Amrita Mohan $^{2}$, Sarah Tabrizi ${ }^{3}$, Albert Robichaud ${ }^{1}$, James Doherty ${ }^{1}$, Michael Quirk $^{1}$

${ }^{1}$ Sage Therapeutics, Pharmacology and Translational Science, Cambridge, MA, USA; ${ }^{2}$ CHDI Foundation, New York, NY, USA; ${ }^{3}$ UCL Institute of Neurology, University College, London, UK

24(S)-hydroxycholesterol (24(S)-HC) is an endogenous, brain specific, cholesterol metabolite that acts as a positive allosteric modulator of the Nmethyl-D-aspartate (NMDA) receptor. Alterations in plasma and/or brain levels of 24(S)-HC have been identified in several diseases, including Smith-Lemli-Opitz syndrome, Niemann Pick, Huntington's disease (HD), and some forms of dementia. Although a broad range of pathology and core symptomology is observed across these different disorders, all manifest some degree of behavioral and psychiatric symptoms. One important question to be addressed is whether $24(\mathrm{~S})-\mathrm{HC}$ is associated with these symptoms and which features are most directly associated with decreased glutamatergic tone.

Cognitive deficits are a hallmark of HD and precede the onset of motor impairments by decades. One of the most consistent cognitive findings in HD are deficits in emotional face processing, which are present in both manifest and pre-manifest HD. Previous work has established that levels of 24(S)-HC are decreased in plasma and brain in HD patients, suggestive of decreased NMDA receptor function. Here, we investigated the relationship between 24(S)-HC and emotional face processing in samples from TRACK-HD, a longitudinal biomarker study of pre-manifest and early stage HD. Plasma samples from the TRACK-HD study (60 control; 60 Pre-HD; $60 \mathrm{HD}$ ) were analyzed for 24(S)-HC via liquid-liquid extraction and analyzed with LC-MS/MS. Regression analysis was then performed between oxysterol levels and performance on the Ekman and Friesen emotion recognition task.

We find that 24(S)-HC levels are positively correlated with performance on an emotional face processing task that is not due to overall neurodegenerative processes. Importantly, we found no relationship between emotional face processing and other oxysterols (25- and 27-HC) suggesting a specific role for central nervous system derived 24(S)-HC. These data support a critical role for NMDA receptor function in negative emotion processing in $\mathrm{HD}$ and identify emotion recognition as a potential clinical endpoint.

Lay Summary: We have identified a peripherally detectable, brain specific, cholesterol metabolite that may reflect altered NMDA signaling in HD. We found significant correlations between this metabolite and a number of cognitive endpoints in HD. Behavioral and cognitive issues often precede the motor symptoms of HD, sometimes by many years, and there are currently no treatments available for non-motor aspects of Huntington's disease. We are currently investigating a novel NMDA therapeutic in HD.

53

Reliability, Feasibility and Validity of a Novel Digital Monitoring Platform Assessing Cognitive and Motor Symptoms in People with Stage I and II Huntington's Disease (HD)

Florian Lipsmeier ${ }^{1}$, Cedric Simillion ${ }^{1}$, Atieh Bamdadian ${ }^{1}$, Anne Smith ${ }^{2}$, Scott Schobel ${ }^{3}$, Christian Gossens ${ }^{1}$, Patrick Weydt ${ }^{4}$, Edward Wild ${ }^{5}$, Michael Lindemann ${ }^{1}$

${ }^{1}$ Roche Pharma Research and Early Development, pRED Informatics, F. Hoffmann-La Roche Ltd., Basel, Switzerland; ${ }^{2}$ Ionis Pharmaceuticals Inc., Carlsbad, CA, USA; ${ }^{3}$ F. Hoffmann-La Roche Ltd., Basel, Switzerland; ${ }^{4}$ University of Ulm Medical Center, Ulm Germany; Current address: Dept. of Neurodegenerative Disease and Gerontopsychiatry/Neurology, University of Bonn Medical Center, Bonn, Germany; ${ }^{5}$ Huntington's Disease Research Centre, UCL Institute of Neurology, London, UK

Longitudinal monitoring of HD signs and symptoms through frequent, home-based testing promises to enhance standard clinical tests that are potentially limited by natural symptom fluctuations, inter-rater variability and subjectivity. A preliminary analysis of the ongoing RG6042 openlabel extension study (OLE) (NCT03342053) demonstrated that frequent remote monitoring yields reliable and valid measures of cognition and motor symptoms in HD when compared with established clinical measures.

The Roche HD digital monitoring platform includes a smartphone application with seven active tests (Symbol Digit Modalities Test [SDMT], Stroop Word Reading, Speeded tapping, Chorea, Balance, U-turn, and 2min walk test) and continuous passive monitoring. This platform is used in an ongoing, natural history study in patients with Stage I/II HD (NCT03664804). A cross-sectional baseline analysis will be conducted. We will extract predefined sensor features (e.g. number of correct 
responses on the SDMT and U-Turn turn speed) from active tests and passive monitoring, as carried out in a cross-sectional baseline analysis with a similar HD population (RG6042 OLE). Features will be averaged from all tests performed over a 2-week period and compared with standard clinical measures at baseline. Intra-class correlation coefficients and Spearman correlations will be used to quantify test-retest reliability, and convergent validity of novel digital measures compared with equivalent in-clinic tests.

We will report on preliminary adherence to demonstrate acceptance and feasibility of the approach and evaluate the test-retest reliabilities of active test features and compare with those collected in a separate study. Results will be reported for correlations of digital test features with related clinical scores.

Further evidence of the feasibility, validity, and reliability and agreement between digital and established clinical measures will support continued use of frequent remote digital monitoring of cognitive and motor symptom severity in patients with HD.

Funding Information: This study is funded by Ionis Pharmaceuticals and F. Hoffmann-La Roche Ltd.

Lay Summary: These investigations aimed to find out if motor and cognitive symptoms in Huntington's disease (HD) could be reliably measured using a series of at-home tests, developed for use on a smartphone application (app). The researchers will investigate whether the information taken from the in-app tests during two studies are as reliable as inclinic assessments of HD. This app has the potential to improve how disease progression is monitored in HD. It could result in more efficient clinical trials and improved routine patient care.

\section{4}

HD 101: Educating Occupational Therapy Practitioners about Huntington's Disease

Deb Lovecky ${ }^{1}$, Debbie Amini ${ }^{2}$, Sandra Kostyk ${ }^{3}$, Ashwini Rao ${ }^{4}$, K.M. "Shelley" Watkins ${ }^{5}$, Cindy Sargent ${ }^{6}$

${ }^{1}$ Huntington's Disease Society of America (HDSA), New York, NY, USA; ${ }^{2}$ American Occupational Therapy Association, Bethesda, MD, USA; ${ }^{3}$ The Ohio State University, Columbus, OH, USA; ${ }^{4}$ Columbia University, New York, NY, USA; ${ }^{5}$ University of Virginia, Charlottesville, VA, USA; ${ }^{6}$ University of Alabama, Birmingham, AL, USA

HDSA currently has 47 HDSA Centers of Excellence (plus 6 partner sites) that provide exemplary medical and social services to HD families including physical, occupational and speech evaluations. However, ensuring access to ongoing community based rehabilitation services has been a challenge. The number of HD experienced therapists in the areas of physical, occupational and speech therapy are limited. Thus, HDSA sought to create strategic partnerships with the most relevant professional organizations to develop continuing education courses that would provide community based therapy specialists with the tools and knowledge needed to evaluate, assess and treat a person with HD.

HDSA formed an alliance with the American Occupational Therapy Association (AOTA) in 2015. Utilizing HD knowledgeable therapists from HDSA Centers of Excellence, a fully-accredited, multi-module course that provided up to four free continuing education credits (CEs) was created to provide essential information about HD. The course was hosted on the AOTA website for a period of three years.

At the completion of the course, participants were tested on knowledge learned in key areas including disease symptomology/progression, evaluation/management of persons with HD throughout the course of the disease, as well as strategies to address special issues pertinent to HD. Over a three-year period, more than 2200 continuing education credits were issued to licensed occupational therapy practitioners by AOTA for the four modules comprising the course.
By educating community-based occupational therapy practitioners, in the specific areas of knowledge needed to assess, evaluate and provide ongoing services, HDSA has improved access to knowledgeable rehabilitation professionals in the community. Partnering with the AOTA, the premier professional organization for licensed occupational therapists, provided HDSA with access to the broadest number of trained OTs in the US. Offering free $\mathrm{CE}$ credits ensured that the maximum number of professionals were exposed to the course and the information provided.

Lay Summary: By educating community-based occupational therapy practitioners in the specific areas of knowledge they will need to assess, evaluate and provide ongoing rehabilitation services, HDSA has improved access to HD knowledgeable professionals in the community. Offering free continuing education credits, which are required for licensing, ensures that the maximum number of professionals are exposed to the course and the information provided.

\section{5}

Improving Access to Care: Educating Community-based Medical Professionals about HD

Deb Lovecky ${ }^{1}$, Jennifer Bomberger ${ }^{2}$, Gena Dolson ${ }^{2}$, Jess Dropkin ${ }^{2}$, Rupinder Hayer ${ }^{2}$, Catherine Murray ${ }^{2}$, Ronan O'Beirne ${ }^{3}$, Jee Bang ${ }^{4}$, Danny Bega ${ }^{5}$, Daniel Claassen ${ }^{6}$, Samuel Frank ${ }^{7}$, Madaline Harrison, Sandra Kostyk ${ }^{9}$, Erin Furr Stimming ${ }^{10}$, Victor Sung ${ }^{3}$, Vaslerie Suski ${ }^{11}$, Claudia Testa ${ }^{12}$

${ }^{1}$ Huntington's Disease Society of America (HDSA), New York, NY, USA; ${ }^{2}$ Medscape, New York, NY, USA; ${ }^{3}$ University of Alabama, Birmingham, AL, USA; ${ }^{4}$ Johns Hopkins University, Baltimore, MD, USA; ${ }^{5}$ Northwestern University, Chicago, IL, USA; ${ }^{6}$ Vanderbilt University, Nashville, TN, USA; ${ }^{7}$ Beth Israel Deaconess Medical Center, Boston, MA, USA; ${ }^{8}$ University of Virginia,24821247571 Charlottesville, VA, USA; ${ }^{9}$ The Ohio State University, Columbus, OH, USA; ${ }^{10}$ University of Texas Health Science Center, Houston, TX, USA; ${ }^{11}$ University of Pittsburgh, Pittsburgh, PA, USA; ${ }^{12}$ Virginia Commonwealth University, Richmond, VA, USA

Overview: Huntington's disease is rare and thus there are a limited number of trained, qualified clinicians in the community. While HDSA has strategically placed 53 HDSA Centers of Excellence in major population hubs, many HD families are unable to or elect not to use one within their region. To that end, HDSA identified a need to educate community based healthcare providers about Huntington's disease in order to address this segment of the HD community that does not currently utilize the services provided by an HDSA Center of Excellence.

The Process: In 2017, HDSA joined with Medscape to create a one year accredited multi-module Continuing Medical Education (CME) course for primary care providers, neurologists and psychiatrists not familiar with HD. The goal was to provide basic knowledge about the disease, its progression and current treatment options so providers who might currently be treating a person with HD in the community, or who might provide care in the future, would have the necessary tools and resources. Experts from HDSA Centers of Excellence assisted in program development and served as presenters/panelists.

Findings: By the end of 2018, 12,294 learners had viewed the three modules with Medscape awarding 5040 continuing education credits. The most surprising outcome was the number of psychiatrists who participated and earned credits. Of the 12,294 learners, 4576 (37\%) were community-based psychiatrists who earned $60 \%$ of the CE credits awarded (3041).

While HDSA continues to expand the number of HDSA Centers of Excellence, in order to provide expert care to more HD patients and families, partnering with experienced medical education providers, like Medscape, expands the network of HD knowledgeable community based providers. Offering the expert knowledge, tools and ongoing resources these medical professionals need remains key to ensuring quality care for all persons affected by HD. 
Lay Summary: While HDSA continues to expand the number of HDSA Centers of Excellence, in order to provide expert care to more HD patients and families, partnering with experienced medical education providers, like Medscape, expands the network of HD knowledgeable communitybased providers. Offering the expert knowledge, tools and ongoing resources these medical professionals need remains key to ensuring quality care for all persons affected by HD.

\section{6}

High Intensity Onabotulinum ToxinA Therapy for the Management of Severe Dystonia in Juvenile-onset Huntington's Disease

Leslie C. Markun, Lin Zhang, Alexandra O. Duffy, A. Joshua Dayananthan, Vicki L. Wheelock

University of California Davis, HDSA Center of Excellence, Sacramento, CA (all authors)

Background: Symptomatic management of dystonia in individuals with Juvenile-onset Huntington's disease can often be difficult and refractory to medication treatment. Few case reports have described symptomatic management with botulinum toxin treatment.

Methods: A single movement disorders neurologist (LZ) performed intramuscular onabotulinum toxinA injections using electromyographic guidance. Standard dosing based on activity of muscle recruitment was selected. Treatment plan adjustments in dosing and frequency were made based on patient response.

Results: Patient 1 was a 20 year-old girl with Juvenile-onset Huntington's disease starting at age 6 years with progressive bradykinesia, rigidity and painful severe dystonic spasms. She had severe arm involvement with forced elbow flexion causing her to have several excoriations of her face. She additionally had retrocollis, lingual and jaw opening dystonia and blepharospasm. She had a partial response to treatment with clonazepam, baclofen and carbidopa/levodopa. She underwent botulinum toxin treatment at targeted muscle groups every 6-8 weeks totaling 600-800 units per treatment. Response with marked improved motor control was noted with six weeks of good effect. She had continued response to treatment for 3 years.

Patient 2 was a 22 year-old girl with Juvenile-onset Huntington's disease starting at age 13 years with progressive rigidity and severe dystonia of her arms bilaterally and severe anterocollis. Her fingers were clutched and passive upper extremity motion was extremely limited. She was treated with trihexyphenidyl and amantadine, but with inadequate symptomatic relief. She underwent botulinum toxin treatment every 6-8 weeks at targeted muscle groups totaling 300-400 units per treatment. Response with improved motor control was noted with eight weeks of good effect. She had continued response to treatment for 6 years.

Conclusions: We report two patients treated with high intensity botulinum toxin injections that resulted in substantial symptomatic relief for acquired dystonia in Juvenile-onset Huntington's disease.

Lay Summary: Symptomatic management of dystonia in individuals with Juvenile-onset Huntington's disease can often be difficult to treat. We describe a technique of high intensity botulinum toxin therapy as a management option.

57

\section{Risky Behaviors in HD Patients: A First Look at a New Measure}

Katherine McDonell ${ }^{1}$, Abagail E. Ciriegio ${ }^{2}$, Lisa Hale ${ }^{1}$, Anna C. Pfalzer ${ }^{1}$, Heather Riordan ${ }^{1}$, Sarah Moroz ${ }^{1}$, Bruce E. Compas ${ }^{2}$, Daniel O. Claassen ${ }^{1}$

${ }^{1}$ The Huntington Disease Society of America Level 1 Center of Excellence at Vanderbilt University Medical Center, Nashville, TN, USA $;{ }^{2}$ Vanderbilt University, Nashville, TN, USA

Cognitive and psychiatric changes associated with Huntington disease (HD) typically emerge early in the disease process and are often the most burdensome for patients and caregivers. One aspect of HD that is commonly observed but not well-studied is a tendency towards risky, impulsive behaviors. These behaviors, including hypersexuality, reckless driving, substance use, and physical aggression, can be extremely detrimental and can lead to adverse personal, social, and legal consequences. However, there is currently little literature regarding these symptoms, and no empirically validated measures are available to screen for them. We present findings on a new measure: The Risky Behavior Questionnaire for HD Patients (41 items, internal consistency reliability = .91 ). In a pilot sample of $13 \mathrm{HD}$ patients (mean age $=44$ ), $64 \%$ of patients endorsed engaging in risky behaviors, further $46 \%$ of patients scored in the moderate to severe range for levels of depression. Levels of depression were significantly correlated with total number of risky behaviors $(r$ $=.65, p=.031)$ and specifically with inappropriate sexual behaviors $(r=$. $75, p=.012)$ and physical abuse of others $(r=.73, p=.011)$. These findings suggest the importance of screening for risky and impulsive behaviors as part of an overall mental health profile of HD patients. Data on a larger sample will be available at the time of conference.

Lay Summary: Risky, impulsive behaviors in HD patients are not wellstudied. We present findings on a new measure: The Risky Behavior Questionnaire for HD Patients. These findings suggest the importance of screening for risky and impulsive behaviors as part of an overall mental health profile of HD patients.

\section{8}

D-Serine and Metabolic Pathways in Huntington's Disease of Varying Severity: A Cross-Sectional Pilot Study

Andrew McGarry ${ }^{1}$, John Gaughan ${ }^{1}$, Ruin Moaddel $^{2}$, Basant Pradhan ${ }^{1}$, Hamza Shaikh ${ }^{1}$, Cory Hackmyer ${ }^{3}$, Irving Wainer ${ }^{4}$

${ }^{1}$ Cooper University Healthcare at Rowan University, Camden, NJ, USA; ${ }^{2}$ National Institute on Aging, Bethesda, MD, USA; ${ }^{3}$ Touro College of Osteopathic Medicine, Middletown, NY, USA; ${ }^{4}$ Chief Science Officer, Mitchell Woods Pharmaceuticals

Introduction: In HD, no treatment exists for cognitive disability. Dserine (DSR), the most biologically active D-amino acid in the brain, plays a role in dendritic development, synaptic plasticity, and neuronal migration. It is a co-agonist of the N-methyl-D-aspartate receptor and predominantly active at synaptic NMDAR, where long-term potentiation and depression are mediated. DSR may be of importance for cognitive function, though little is known about DSR biology in HD. Here we report initial data in a cross-sectional study of HD participants of varying severities, and explore the relationship between other metabolites and disease severity as measured by the UHDRS.

Methods: 12 symptomatic participants with genetically confirmed HD of varying severity (TFC 3-13) underwent plasma draw and lumbar puncture. Metabolites were analyzed using the AbsoluteIDQ kit p180 (Biocrates Life Science AG, Austria) and the Lipidyzer Platform ((Sciex, Framingham, MA) on a 5500 QTrap(r) (Sciex) mass spectrometer. D-serine was measured following previously developed protocols.

Results: Plasma DSR showed a significant correlation with Stroop Word task and associations with SDMT and independence Scale scores, while total serine showed a significant correlation with Stroop Color and Interference tasks. In CSF, D-serine was not significantly correlated with disease progression; significant correlations included asparagine (positive) and arginine (negative). Arginine, citrulline, and glycine in plasma demonstrated negative correlations with cognitive outcomes. CSF creatinine displayed negative correlations across numerous indices of disease severity, while normal in plasma. A number of triacylglyceride and sphingomyelin species were negatively correlated with disease progression.

Discussion: In this pilot study, decreasing DSR in plasma correlates with cognitive decline. Plasma arginine and glycine were negatively correlated with cognitive decline, with glycine positively correlated with TFC progression. Further study is warranted to determine the natural history and 
therapeutic potential for modification in HD of DSR, arginine, glycine, and lipid species.

Lay Summary: In HD, no treatment exists for cognitive disability. Understanding D-serine, an amino acid in the brain thought to be involved in neuronal plasticity and cognitive function, may reveal strategies for the development of new therapies.

59

Baseline Variables Associated with Functional Decline in 2CARE, A Randomized Clinical Trial in Huntington's Disease

Andrew McGarry ${ }^{1}$, Michael P. McDermott ${ }^{2}$, Karl Kieburtz ${ }^{2}$, Jing Peng ${ }^{3}$, Merit Cudkowicz ${ }^{4}$, for the Huntington Study Group 2CARE Investigators and Coordinators

${ }^{1}$ Cooper University Healthcare at Rowan University, Camden NJ, USA; ${ }^{2}$ University of Rochester, Rochester, NY, USA; ${ }^{3}$ The Ohio State University, Columbus, OH, USA; ${ }^{4}$ Harvard Medical School, Boston, MA, USA

Introduction: Huntington's disease (HD) is a progressive, fatal neurodegenerative disorder involving motor, cognitive, and behavioral abnormalities that exert a cumulative effect on daily function. It is not clear in the literature which demographic and lifestyle factors are most related to functional progression over time. Understanding variables that are associated with functional decline may be of value towards improving management strategies and quality of life. Using data from 2CARE, a large randomized clinical trial that included up to 5 years of follow-up of participants, we sought to identify baseline clinical, demographic and lifestyle factors that are associated with functional decline as measured by the TFC.

Methods: The outcome variable in this secondary analysis was rate of change over time (slope) for the TFC score. Potential baseline risk factors for more rapid progression were examined using linear mixed effects models that included TFC score as the outcome variable and the risk factor, time (continuous, expressed as the number of years since randomization), and the interaction between the risk factor and time as independent variables.

Results: Variables associated with faster functional decline included worsening motor performance, worsening cognitive test scores, bipolar disorder, female sex, weight and body mass index, CAG repeat length, and an interaction between CAG repeat length and age.

Conclusion: Attention to several of the identified risk factors may be useful towards managing functional decline over time. The finding that women experienced a more rapid functional decline than men, an observation that did not have an interaction with weight/body mass index, deserves attention. It is not clear what the biological basis for this asymmetry would be, as sex hormones are not thought to be relevant to disease mechanisms; this finding requires further study to determine if sex is associated with functional preservation in some currently unknown way. Lay Summary: While the importance of preserving function is generally accepted as an aim of clinical care in HD, it is not entirely clear what factors are most related to functional progression over time. Understanding clinical, demographic, or lifestyle features that are associated with functional decline may be of value towards improving management and quality of life.

\section{0}

Difficulties of Speech and Swallowing in Colombian Population With Huntington's Disease

Maryluz Camargo Mendoza

Universidad Nacional de Colombia, Bogotá, Colombia

Background: Difficulties in speech and swallowing are very common in people with Huntington's Disease and may appear earlier than other motor or psychiatric symptoms characteristic of the disease. These alterations have been described as a result of the choreic movements that arise from the dysfunction of the basic ganglia and that affect the way in which the musculature implies the proper functioning of speech and swallowing structures.

Purpose: To describe the speech and swallowing characteristics of people with Huntington's Disease in two main areas of Colombia.

Methods: Data were taken from 20 people from two different regions of Colombia through two questionnaires, one dedicated to determining the characteristics of speech, and the other, to the characteristics of swallowing using the Munich Dysphagia Test -MDT-

Results: The results show that, on the one hand, the main difficulties that people report with respect to their speech are: running out of breath when talking, decreasing the volume of the voice and the sensation of having a hoarse or variable voice with tension in the muscles of the neck, difficulty for others to understand their productions, as well as a self-perception of speaking entangled and more slowly. On the other hand, the difficulties of swallowing are related to the escape of food through the mouth or nose, whole swallowing sensation, residues in the mouth after passing food and need to clear your throat or cough during or after eating.

Conclusion: Problems in speech and swallowing of the people studied are present with different degrees of severity depending on the stage they are in HD. In early stages with signs that do not compromise speech intelligibility or the safe passage of food, and in advanced stages where the production of speech is very altered or is no longer, and oral feeding compromises the safety of the airway.

Lay Summary: This study is important for the HD community because it shows difficulties in speech and language in people with HD at different stages of the disease, which can contribute to understanding the disease much more as well as providing information to undertake therapeutic actions.

\section{1}

\section{Multi-Step Prescreening Strategies for HD-Trials}

Sarah Moroz, Danielle Buchanan, Brittany Brown, Daniel Claassen Vanderbilt University Medical Center, Nashville, TN, USA (all authors)

Background: The initiation of disease-modifying therapies for Huntington's disease has garnered substantial interest in patient communities. We have noted an influx of patient calls for participation in these trials. To address this, we implemented a multi-step recruitment model, similar to those previously reported, with the objectives to ensure high recruitment, low screening failure and dropout rates, as well as to adequately inform patients of trial opportunities and manage their expectations.

Methods: This multi-step screening process utilizes internal and observational databases to identify patients who meet inclusion and exclusion criteria for studies. We notate families who have expressed specific interest in participating in clinical trials, including those who have reached out to us from outside the VUMC network. These patients, as well as their primary caregivers, are contacted by phone and interviewed to establish eligibility, commitment, and feasibility (i.e. travel logistics). For queries from patients outside our clinic, we require establishing care at our center to begin a clinical relationship with the HD team. Patients are then placed into a pool of potential study participants and contacted based on the lead coordinator's discrimination.

Results: Since initiation of the multi-step screening process, we have had over 100 patient inquiries, scheduled at least 50 new patient visits, and successfully enrolled 39 patients in 3 disease modifying studies. Our site reports a low number of screening failures and study drop-outs. Through this process, we also noticed the need for inter-site communication, generating referrals between 5 other HD sites and thus creating a network through which HD patients and their families can more comfortably seek treatment and access to clinical trials. Conclusion: As a result of this process, we have effectively succeeded in managing recruitment schedules as well as patient expectations. 
Clinicians, social workers, and coordinators have all played an integral role in successfully executing the multi-step screening process.

Lay Summary: With the inception of a number of disease-modifying therapies for Huntington's disease, our clinic has received a significant increase in calls and emails from individuals with HD, or their family members, who are interested in participating. In order to effectively communicate the requirements of the trials, as well as to manage patient expectations and decrease the likelihood of screening failures and study drop-outs, we have implemented a multi-step prescreening procedure at our clinic. This system has allowed clinicians, coordinators, and social workers to establish better relationships with patients - old and new and improved screening outcomes for a number of HD trials.

62

Profiling Microglial Phenotypes in HD Using Flow Cytometry and Cluster Mapping

Scott D. Olson, Naama Toledano Furman, Karthik Prabhakara, Erin Furr Stimming, Amit Srivastava

University of Texas Health Science Center at Houston, Houston, TX, USA (all authors)

Neuroinflammation has been shown to be a significant component of the development of Huntington's disease (HD). This inflammation of the CNS leads to chronic activation of microglia, which contributes to neurodegeneration. A number of interventions have been proposed to target microglial activation to reduce neuroinflammation as a treatment for HD. However, the study of microglia has presented several formidable technical challenges, including difficulty in differentiating microglia from invasive peripheral monocytes and macrophages responding to CNS inflammation. Historically, microglial activation has been described using polarized M1 and M2 phenotypes, however more recent work has found this convention to be oversimplified and insufficient to address the dynamic nature of microglial responses to stimuli in vivo. For these reasons, we have applied new multidimensional cluster-based flow cytometry analysis techniques to a multicolor panel featuring microglial surface markers. These techniques are used to map and discover clusters of events across individuals using unbiased comparisons of surface phenotype marker presence and expression levels. When combined with conventional logic-gating based analysis, these new techniques more accurately demonstrate the changes in microglial phenotypes and conventional M1/M2 surface markers in HD. This will be useful in detecting mild or modest effects of therapeutic interventions on microglia.

Lay Summary: This work is important to understanding the changes in the immunobiology of the brain, and specifically microglia, as a result of HD. In recent years, microglial biology has undergone a significant shift to better understand the role of dynamic and mixed pro- and antiinflammatory signals and activities that occur inside the CNS as a response to injury and disease. Here we are applying newly developed techniques to better analyze the changes that are occurring in microglia in an R6/2 animal model of HD.

63

Cost of Predictive Testing in HD Varies Greatly Across Centers of Excellence in the United States

Tyler Orem, Victor Sung

University of Alabama at Birmingham, Birmingham, Alabama, USA (all authors)

Background: Huntington's disease (HD) is a genetically determined neurodegenerative disorder. Despite the availability of pre-symptomatic (predictive) genetic testing, most at-risk individuals choose not to undergo testing. With many new therapies for HD on the horizon, it is important to identify factors contributing to the decision to test for atrisk individuals. There has been insufficient data published on cost of testing as a factor. Therefore, we sought to determine whether the cost of predictive testing is a factor in the decision to test in the United States. Methods: The Huntington's Disease Society of America (HDSA) provided de-identified data from individual Centers of Excellence (COEs) on the various components of the predictive genetic testing process at each $\mathrm{COE}$ as well as the cost of each component for an uninsured patient. This data was self-reported by each $\mathrm{COE}$ as part of an annual progress report. Data from 2017 (28 COEs) and 2018 (19 COEs) progress reports were aggregated and analyzed.

Results: In 2017, the average cost of a predictive genetic test was $\$ 1128$ (range \$324-\$3640). In 2018, the average cost of the genetic test was $\$ 1142$ (range \$250-\$2625). Only 37\% of COEs reported the availability of financial assistance in 2017, and only $39 \%$ of COEs reported the availability of financial assistance in 2018

Conclusions: Even at COEs, which perform predictive HD genetic testing with greater regularity than a private practitioner, there is great variability in the cost of testing. With costs averaging $>\$ 1000$, high costs could play a role in the decision to seek pre-symptomatic genetic testing. Future research should closely examine the impact of high costs on the decision-making process of individuals at-risk for HD.

Lay Summary: With many new therapies for HD on the horizon, it is important to identify factors contributing to the decision to test for at-risk individuals. Our research seeks to determine if there is a financial barrier preventing many at-risk individuals from undergoing predictive testing.

\section{4}

Brain-Derived Neurotrophic Factor in Cerebrospinal Fluid and Plasma as a Potential Biomarker for Huntington's Disease

Zhen-Yi Andy $\mathrm{Ou}^{1}$, Lauren M. Byrne ${ }^{1}$, Filipe B. Rodrigues ${ }^{1}$, Rosanna Tortelli $^{1}$, Eileanor B. Johnson ${ }^{1}$, Martha S. Foiani ${ }^{4}$, Marzena Arridge ${ }^{2}$, Enrico De Vita ${ }^{2,3}$, Rachael I. Scahill ${ }^{1}$, Amanda Heslegrave ${ }^{4}$, Henrik Zetterberg $^{4,5,6}$, Edward J. Wild ${ }^{1}$

${ }^{1}$ Huntington's Disease Centre, University College London (UCL) Institute of Neurology, London WC1N 3BG, UK; ${ }^{2}$ Lysholm Department of Neuroradiology, National Hospital for Neurology and Neurosurgery, London WC1N 3BG, UK; ${ }^{3}$ Department of Biomedical Engineering, School of Biomedical Engineering and Imaging Sciences, King's College London, London SE1 7EH, UK; ${ }^{4}$ Fluid Biomarker lab, UK Dementia Research Institute at UCL, London WC1E 6BT, UK; ${ }^{5}$ Department of Psychiatry and Neurochemistry, Institute of Neuroscience and Physiology, Sahlgrenska Academy at the University of Gothenburg, Mölndal, 40530 Gothenburg, Sweden; ${ }^{6}$ Clinical Neurochemistry Laboratory, Sahlgrenska University Hospital, Mölndal, 41345 Gothenburg, Sweden

Brain-derived neurotrophic factor (BDNF), a member of the neurotrophin family, is implicated in the survival of striatal neurons. BDNF function is reduced in Huntington's disease (HD), possible because mutant huntingtin impairs its transport from cortex to striatum, which may contribute to dysfunction and degeneration of striatal neurons. BDNF in blood has been suggested as a potential biomarker for HD, but BDNF has not been quantified in cerebrospinal fluid (CSF) in HD.

Our aims were to quantify BDNF in CSF and blood plasma to assess its potential as a biomarker for disease progression in HD.

We quantified BDNF in CSF and plasma in the HD-CSF cohort, consisting of 20 pre-manifest and 40 manifest HD mutation carriers and 20 age- and gender-matched controls using an ultrasensitive immunoassay (Quanterix ${ }^{\circledR}$ SimoaTM). We also evaluated several conventional BDNF ELISA kits used in previous CSF reports. Statistical analyses included multiple regression, ANOVA, and ROC analysis. Results were Bonferroni-corrected and adjusted for age, gender, BMI, anti-depressant, anti-psychotic, and blood platelet count or CSF erythrocyte count. 
BDNF concentration did not differ significantly between controls and HD mutation carriers in CSF or plasma. Plasma and CSF BDNF in controls, premanifest and manifest HD did not differ across groups (ANOVA $P=$ 0.1058 and $P=0.6564$ respectively). BDNF in plasma and CSF were not associated with clinical or MRI brain volumetric measures. Plasma and CSF BDNF had poor diagnostic ability in discriminating controls from HD mutation carriers (plasma $\mathrm{AUC}=0.494, \mathrm{CSF} \mathrm{AUC}=0.602$ ) and premanifest from manifest $\mathrm{HD}$ (plasma $\mathrm{AUC}=0.409, \mathrm{CSF} \mathrm{AUC}=$ $0.454)$.

CSF BDNF concentrations fell below the lower limit of quantification of all tested ELISA kits used in previous reports in neurodegeneration.

We conclude that BDNF in both CSF and plasma is not a biomarker of progression in $\mathrm{HD}$, and urge caution in interpreting studies where conventional ELISA was used to quantify CSF BDNF.

Lay Summary: Brain-derived neurotrophic factor, a neuronal growth protein produced by the brain, is reduced in HD and its level in blood or cerebrospinal fluid (CSF) has been suggested as a way of measuring or predicting progression. Using an ultra-sensitive measurement platform, we show that BDNF level is not altered in blood or CSF at any stage of $\mathrm{HD}$, and that the methods previously used in other brain diseases were probably not sensitive enough to measure it reliably in CSF.

\section{5}

National Trends in Inpatient Gastrostomy Placement for Huntington Disease

Meredith Pauly ${ }^{1}$, Dylan P. Thibault ${ }^{2}$, Pedro Gonzalez-Alegre ${ }^{3,5}$, Allison W. Willis 2,3,4, $^{\text {, Ali G. Hamedani }}{ }^{3,4}$

${ }^{1}$ Good Shepherd Penn Partners, Penn Therapy and Fitness, Pennsylvania Hospital, Philadelphia, PA, USA; ${ }^{2}$ Department of Biostatistics and Epidemiology, Center for Clinical Epidemiology and Biostatistics, University of Pennsylvania, Philadelphia, PA, USA; ${ }^{3}$ Department of Neurology, Perelman School of Medicine, University of Pennsylvania, Philadelphia, PA, USA; ${ }^{4}$ Translational Center of Excellence for Neuroepidemiology and Neurology Outcomes Research, University of Pennsylvania, Philadelphia, PA, USA; ${ }^{5}$ Raymond G. Perelman Center for Cellular \& Molecular Therapeutics, The Children's Hospital of Philadelphia, Philadelphia, PA, USA; ${ }^{6}$ Leonard Davis Institute of Health Economics, University of Pennsylvania, Philadelphia, PA, USA

Background: HD causes dysphagia and dementia, both of which are risk factors for poor oral intake and malnutrition. Gastrostomy is used to sustain enteral intake in patients with neurodegenerative disease, but the frequency and associated outcomes in HD are unknown.

Methods: We performed a retrospective cross-sectional analysis of data from the National Inpatient Sample, sampling all inpatient admissions with a diagnosis of HD and separating those with and without a claim for gastrostomy placement. We used logistic regression to compare the patient- and hospital-level characteristics associated with gastrostomy placement in HD and compared the prevalence of associated diagnoses in HD gastrostomy patients to ALS gastrostomy patients. We also examined outcomes of in-hospital mortality, length of stay, and discharge status.

Results: Of 31,636 inpatient admissions with a diagnosis of HD from 2004 to 2013, $1694(5.35 \%)$ were associated with gastrostomy tube placement. Gastrostomy patients were more likely to be Black (adjusted odds ratio [AOR] 1.55, 95\% CI: 1.09-2.21) or Native American (AOR 3.47, 95\% CI: $1.21-9.95$ ) and more likely to have Medicare coverage (AOR 1.43, 95\% CI: 1.0-2.05). The most common associated diagnoses were aspiration pneumonia $(34.1 \%)$, dementia $(31.3 \%)$, malnutrition $(30.3 \%)$, and dysphagia (29.5\%). Compared to inpatient gastrostomy patients with ALS, those with HD had significantly greater odds of aspiration pneumonia $(34.1 \%$ vs. $20.5 \%, p<0.0001)$ and sepsis $(28.1 \%$ vs. $13.7 \%, \mathrm{p}<0.0001)$ and had longer length of stay (OR 1.14, 95\% CI: $1.02-1.28)$ and greater skilled nursing facility discharge $(\mathrm{p}<0.0001$ for
Wald chi square test). Among HD gastrostomy patients, dementia and delirium were associated with non-routine discharge but not length of stay, and aspiration pneumonia, sepsis, and Elixhauser comorbidity index were associated with length of stay but not discharge status.

Conclusion: Dementia and aspiration pneumonia are key determinants of gastrostomy placement in hospitalized HD patients and are associated with negative hospitalization outcomes.

Lay Summary: Gastrostomy tubes are used to sustain enteral intake in patients with neurodegenerative disease, but the frequency with which this occurs in HD and its associated risk factors and outcomes are unknown. Identifying this will guide clinicians' conversations with people who have $\mathrm{HD}$ and their families in order to support treatment decision-making.

66

Use of the Enroll-HD Observational Study as a Platform for Enriching Data Collection in a 12-month Nested Randomized Controlled Feasibility Study of a Physical Activity Intervention in People with Huntington's Disease (PACE-HD)

Cheney J Drew ${ }^{1}$, Olivia Handley ${ }^{2}$, Jenny Townhill ${ }^{2}$, Rhys WilliamsThomas $^{1}$, Monica Busse ${ }^{1}$, Lori Quinn ${ }^{1,3}$

${ }^{1}$ Centre for Trials Research, Cardiff University, Cardiff, UK; ${ }^{2}$ European Huntington Disease Network, University of Ulm, Germany ${ }^{3}$; Teachers College, Columbia University, New York, NY, USA

Background: PACE-HD is a multi-site, 12-month trial within a cohort evaluation of a physical activity intervention in people with Huntington's Disease with a target recruitment of $n=120$. The study design incorporates collection of assessment data at baseline and 12 months, supplemented by outcome data collected at participants' annual Enroll-HD visit.

Methods: Trial sites were selected based on information provided in feasibility forms and numbers recruited to Enroll-HD, facilitated by the Enroll-HD clinical trials manager. Following IRB approval, sites were provided with details of potentially eligible participants according to study inclusion/exclusion criteria by the Enroll-HD clinical trials manager. Further screening for eligibility and recruitment was conducted at site. A specific data request was approved by the EnrollHD scientific planning committee to provide supplementary outcome data at specified timepoints. Lastly, a monitoring strategy utilizing Enroll-HD monitors to confirm veracity of consent and linking of Enroll-HD and PACE-HD identifiers for data linkage was devised in conjunction with the Enroll-HD team.

Results: 411 potential participants were identified via Enroll-HD records. Sites reported screening a total of 273 participants for inclusion on the trial, culminating in the recruitment of 116 participants. Enroll-HD monitors were able to visit each site at least once to ensure verification of eligibility, consent and linked participant identifiers, with completion of monitoring triggering the generation of the baseline data set. $88 \%$ of baseline assessments were performed within the stipulated 4-week window from the participant's annual Enroll assessment.

Conclusions: The use of a global observational platform study such as Enroll-HD can be used successfully to deliver clinical trial research in terms of site selection, recruitment, site monitoring and data collection. The use of platforms like Enroll-HD for data collection in future studies has the potential to support innovative trial designs and reduce the burden on research participants.

Lay Summary: PACE-HD is a multi-site, 12-month trial within-cohort evaluation of a physical activity intervention in people with HD being conducted across 3 countries. Use of the Enroll-HD platform has been essential in coordinating site selection, recruitment, site monitoring and data collection, and has reduced burden on research participants. The use of a global observational platform study such as Enroll-HD can be used successfully to deliver clinical trial research incorporating innovative trial designs such as PACE-HD. 
67

Piloting Data Linkage with the Enroll-HD Dataset: Linking Between Enroll-HD and PACE-HD

Rhys Williams-Thomas ${ }^{1}$, Cheney Drew ${ }^{1}$, Fransisco Quevedo Fernandez ${ }^{1}$, Katie Taiyari ${ }^{1}$, Rebecca Playle ${ }^{1}$, Mette Gilling ${ }^{2,3}$, Lori Quinn $^{1,4}$, Monica Busse ${ }^{1}$

${ }^{1}$ Centre for Trials Research, Cardiff University, Cardiff, UK; ${ }^{2}$ The European Huntington's Disease Network, University of Ulm, Ulm, Germany; ${ }^{3}$ University of Copenhagen, Copenhagen, Denmark; ${ }^{4}$ Teachers College, Columbia University, New York, USA

Background: PACE-HD is a cohort study with a nested randomized pragmatic controlled trial to evaluate a one-year physical activity and exercise intervention in people with Huntington's disease (HD). The PACE-HD study sites also participate in Enroll-HD, a worldwide observational study of HD. For efficiency, a large proportion of trial data will be extracted from Enroll-HD and linked to outcome data collected for PACE-HD. PACE-HD is the first study to request large-scale, specific participant level data from the Enroll-HD study for linkage.

Methods: Variables to be included in the dataset were agreed with the Enroll-HD Scientific Review Committee via the Enroll-HD specified data request process. A dummy dataset of agreed variables was produced to allow preparation of the structured query language server (SQL) database for linkage. Upon import, the database reads the uploaded file, populates the data into tables and produces reports on uploaded versus expected data types and ranges. The dummy dataset enabled testing of this functionality before receiving live data. Data for active study participants was requested from Enroll-HD when all PACE-HD study sites had recruited at least five participants. No identifiable data is collected as part of either study dataset, PACE-HD site staff were required to maintain look-up tables matching unique identifiers for Enroll-HD (HDID) and PACEHD (PID) to enable linkage by the Enroll-HD team.

Results: The pilot dataset was imported and linking functionality of the PACE-HD database fully tested.

Conclusions: We have formalized a data linkage model using a specified dataset from the Enroll-HD study to be used for PACE-HD. This validated linkage procedure will enable us to import future study data without the need for further data checks thus improving trial efficiency. Importantly linkage to a global observational study has the potential to reduce participant burden and has implications for wider use in future HD trials.

Lay Summary: PACE-HD, a within-cohort, one-year physical activity and exercise intervention in people with $\mathrm{HD}$, is the first study to request large-scale specific participant level data from the Enroll-HD study for linkage. We have formalized a data linkage model using a specified dataset from the Enroll-HD study to be used for PACE-HD. This validated linkage procedure will enable us to efficiently import future study data and has implications for wider use in future HD trials.

68

Making Sense of Wearable Sensors: Associations Between Movement Smoothness Derived from Wearable Sensors and the UHDRS Chorea Subscore

Gregory Youdan $\mathrm{Jr}^{1}{ }^{1}$, Lisa Muratori ${ }^{2}$, Nora Fritz ${ }^{3}$, Xueyao $\mathrm{Li}^{1}$, Tanya Gurevich $^{4,7}$, Noit Inbar ${ }^{4}$, Inbar Hillel ${ }^{4}$, Monica Busse ${ }^{5}$, Jeffrey M. Hausdorff ${ }^{4,6,7}$, Lori Quinn ${ }^{1,5}$

${ }^{1}$ Teachers College, Columbia University, New York, NY, USA; ${ }^{2}$ Stony Brook University, Stony Brook, NY, USA; ${ }^{3}$ Wayne State University, Detroit, MI, USA; ${ }^{4}$ Tel Aviv Sourasky Medical Center, Israel; ${ }^{5}$ Centre for Trials Research, Cardiff University, Cardiff, UK; ${ }^{6}$ Rush University Medical Center, Chicago, USA; ${ }^{7}$ Tel Aviv University, Israel

Background: Chorea, a hallmark sign of Huntington's disease (HD), is typically assessed using subjective ratings from the Unified Huntington's
Disease Rating Scale (UHDRS). Wearable sensors are poised to provide precise measurement of motor impairments in people with $\mathrm{HD}$; however, objective metrics that reflect chorea have not yet been identified.

Methods: 43 individuals with early to mid-stage HD (mean age: 53.6 years; mean total motor score (TMS): 40.6) sat (30 s) and stood $(45 \mathrm{~s})$ with feet apart and feet together, wearing 6 APDM inertial sensors. Pearson's correlations were performed between jerk (derivative of acceleration that reflects smoothness of movement) of postural sway and UHDRS chorea subscore, total functional capacity (TFC) and TMS. Variables from the wearables (e.g., jerk, total sway area) from all conditions were entered into a stepwise regression model to predict UHDRS chorea subscore. Lowest akaike information criterion (AIC) was used to determine the final model.

Results: Larger jerk of seated postural sway moderately correlated with larger TMS $\left(\mathrm{r}^{2}=0.39\right)$, and weakly correlated with lower TFC $\left(\mathrm{r}^{2}=\right.$ $-0.21)$. Larger jerk of postural sway while standing feet together $\left(r^{2}=\right.$ $0.38)$ and jerk of seated postural sway $\left(r^{2}=0.54\right)$ were moderately correlated with greater UHDRS chorea subscore. The final model determined that total sway area in standing feet apart and jerk of seated postural sway were the best predictors of UHDRS chorea subscore, explaining $36 \%$ of the variance.

Conclusions: Choreic movements lead to an unsmooth movement of the center of mass that can be captured during sitting and standing. Jerk of postural sway from wearable sensors may represent a sensitive measure that relates to chorea intensity and frequency. Additional research is needed to assess the reliability and sensitivity to change of this putative measure of chorea that may be useful in clinical trials.

Lay Summary: Chorea is a hallmark sign of HD; however, it is difficult to reliably quantify. Measures from wearable sensors, specifically those related to the smoothness of postural sway, were found to be correlated with subjective measures of chorea from the UHDRS. Wearable sensors may provide quantitative assessment of the intensity and frequency of chorea, although additional research is needed to assess their use as an outcome measure in clinical trials.

\section{9}

Patient and Physician Perspectives on the Care and Assistance Needs in Huntington's Disease

Karina Raimundo ${ }^{1}$, Ruoding $\operatorname{Tan}^{1}$, Tu My To ${ }^{1}$, Jonathan de Courcy ${ }^{2}$, Umang Ondhia ${ }^{3}$, Hugh Rickards ${ }^{4}$, Martha Nance ${ }^{5}$

${ }^{1}$ Genentech Inc., South San Francisco, CA, USA $;{ }^{2}$ Adelphi Real World, Bollington, Cheshire, UK; ${ }^{3} \mathrm{~F}$. Hoffmann-La Roche Ltd., Basel, Switzerland; ${ }^{4}$ University of Birmingham, Birmingham, UK; ${ }^{5}$ Struthers Parkinson's Centre, Golden Valley, MN, USA

Huntington's disease (HD) is a genetic, neurodegenerative and ultimately fatal disease that leads to loss of independence. Eventually, most HDaffected individuals require assistance with daily activities; however, little is known about the type of care they require, or differences in care requirements across countries.

This is a retrospective analysis of data from the Adelphi HD Disease Specific Programme - a cross-sectional survey of neurologists, patients and care partners conducted in France, Germany, Italy, the UK and the US between July and October 2017. Here we describe patient- and neurologist-reported care and assistance.

Data were available for 1050 patients. 555 (52.9\%) were reported by the physician to have a care partner. The most common physician-reported types of assistance were help with managing finances (41.1\%), emotional support (39.8\%), motivation to engage in daily activities (36.5\%), planning and organizing everyday activities $(34.3 \%)$ and preparing meals $(33.2 \%)$. For patients with a care partner, assistance types were ranked in a similar order at higher rates. The most common needs varied across countries (France: shopping, 42.4\%; Germany: daily activities, $62.4 \%$, Italy: meal preparation, $40.9 \%$; UK: finance management, $42.6 \%$; US: 
emotional support, 37.6\%). Neurologist-perceived disease severity distribution was similar in Italy, UK and the US. Germany had the highest number of mild patients and France the highest number of severe patients. Of 52 patients who responded with care-giving needs, 40 (76.9\%) reported needing assistance and $26(65.0 \%)$ reported receiving assistance. Assistance needs of people with HD vary with disease severity, cultural differences, family support and other factors and a high proportion of individuals are not receiving the assistance they require. A multidisciplinary group can help patients with HD and their families to access appropriate care based on their individual needs.

Funding Information: This study was funded by Genentech Inc. and F. Hoffmann-La Roche Ltd.

Lay Summary: This survey looking at the assistance needs of people living with Huntington's disease (HD) in the US and Europe found that the level of support needed depends on factors such as severity of disease, country of residence and available family support. The survey also found that a high proportion of people living with HD do not receive appropriate support. Findings suggest that having support from a team of different healthcare professionals (e.g. specialist doctors, occupational therapists, nurses) could help people affected by HD to access care for their individual needs.

70

Impact on Work Status of People Caring for Someone with Huntington's Disease

Karina Raimundo ${ }^{1}$, Ruoding Tan ${ }^{1}$, Tu My To ${ }^{1}$, Jonathan de Courcy ${ }^{2}$, Umang Ondhia ${ }^{3}$, Hugh Rickards ${ }^{4}$, Martha Nance ${ }^{5}$

${ }^{1}$ Genentech Inc., South San Francisco, CA, USA $;{ }^{2}$ Adelphi Real World, Bollington, Cheshire, UK; ${ }^{3} \mathrm{~F}$. Hoffmann-La Roche Ltd., Basel, Switzerland; ${ }^{4}$ National Centre for Mental Health, Birmingham, UK; ${ }^{5}$ Struthers Parkinson's Centre, Golden Valley, MN, USA

Huntington's disease (HD) is a genetic, progressive and ultimately fatal neurodegenerative disease that leads to loss of independence. All people with HD eventually require care; however, little is known about the impact caregiving has on a family member's employment status.

This is a retrospective analysis of data from the Adelphi HD Disease Specific Programme, a one-time survey between July and October 2017 of physicians, patients and care partners in France, Germany, Italy, the UK and the US. Since only a small number of care partners answered the survey, we focus on the physician-reported impact of caregiving on employment status.

Data were collected from 1050 patients. Rates of caregiving were higher in France $(69.4 \%)$ and Germany $(64.9 \%)$, while the lowest rates were reported in the US (39.6\%). $555(52.9 \%)$ patients had care partners who were; professional only $(n=68,12.3 \%)$ both professional and nonprofessional $(n=106,19.1 \%)$ or non-professional only $(n=378$, $68.1 \%)$. Among non-professional care partners, the most common family member to fulfill the role was the partner/spouse (74.3\%). $27.4 \%$ of care partners worked full-time, $22.7 \%$ part-time, $22.5 \%$ were retired and $20.1 \%$ were unemployed/homemakers. Germany $(75.0 \%)$ and US $(61.0 \%)$ reported the highest number of full- or part-time care partners, whereas UK reported the lowest rate (15.2\%). Physician-reported impact of patients' HD on the care partner's work status was evaluated in $82.8 \%$ of cases. $45.7 \%$ of care partners reported a change in work status due to HD. Work status changes typically occurred when patients were in Stage II or III.

Significant indirect cost exists for caring for patients with HD. Care partners are usually close relatives and caregiving often has a negative impact on the care partner's employment status, with severity of impact depending on factors including HD stage.

Funding Information: This study was funded by Genentech Inc. and F. Hoffmann-La Roche Ltd.
Lay Summary:All people living with Huntington's disease (HD) eventually require care. In this study, data collected from doctors found that being a carer for a person affected by HD negatively impacts the carer's employment status. Nearly half of carers reported a change in work status, with changes typically occurring when the people they were caring for were in Stage II or III of HD.

71

Predictors of Energy Expenditure During Physical Activity and Exercise in Huntington Disease

Ashwini K. Rao, Simone Norris, Jackie Montes, Paula Wasserman, Karen S. Marder

Vagelos College of Physicians and Surgeons, Columbia University, New York, NY, USA (all authors)

Introduction:People with Huntington disease (HD) have unintended weight loss, which may be due to a deficit in energy expenditure (EE). While resting EE is comparable to healthy controls, EE during physical activity (PA) and exercise are not known. We examined EE during PA and exercise and predictors of EE.

Methods: We tested $10 \mathrm{pHD}($ mean $=43.2 \mathrm{y}), 10 \mathrm{mHD}($ mean $=52.5 \mathrm{y})$, and 7 controls (mean $=46.6 \mathrm{y}$ ). EE was measured during PA (sit, stand, walk at different speeds) and 6-min walk test with an accelerometer system (outcomes: EE, speed, variability). Exercise capacity was measured with cardiopulmonary exercise test (outcomes: Peak $\mathrm{O} 2$ consumption (VO2), heart rate, workload). Predictors of EE included disease severity (UHDRS motor score), lean body mass (measured with Bioelectrical Impedance Analysis), caloric intake (Food Frequency questionnaire), overall PA (Actigraph sensor), gait variability (accelerometer system), and in-vivo muscle oxygen uptake (near infrared spectroscopy). Clinical measures were analyzed with one-way ANOVA. Maximal and sub-maximal exercise data were analyzed using factorial ANOVA. Regression analysis examined predictors of EE, adjusting for age.

Results: Age, lean body mass, and overall PA were similar across groups $(p>0.05)$. EE during sit and stand were similar across groups. EE was higher for $\mathrm{pHD}$ and $\mathrm{mHD}$ while walking at preferred and fasters speeds $(p<0.006)$ and during 6MWT despite walking slower than controls. Peak $\mathrm{VO} 2$ was lower for $\mathrm{pHD}$ and $\mathrm{mHD}$ at $75 \%$ and maximal intensity $(p<$ 0.01 ). Muscle oxygen uptake was lower in pHD and mHD only at peak exercise intensity $(p<0.03)$.

Conclusion: EE deficit is pronounced in $\mathrm{pHD}$ and $\mathrm{mHD}$ during higher intensity of PA and exercise. Disease severity, motor variability and muscle oxygen uptake were predictive of EE deficit. The results suggest that exercise training at intensity less than $75 \%$ may prevent exacerbation of muscle oxidative deficits in people with HD.

Lay Summary: Muscle weakness, reduced exercise capacity and unintended weight loss are commonly seen in Huntington disease (HD), and may be due to increased energy expenditure. Exercise may be beneficial, but may worsen energy expenditure - it is therefore important to understand factors related to energy expenditure during physical activity.

72

\section{Falls and Life-space Mobility in Huntington Disease}

Ashwini K. Rao ${ }^{1}$, Gregory Youdan $\mathrm{Jr}^{2}$, Xueyao $\mathrm{Li}^{2}$, Nora Fritz ${ }^{3}$, Lisa Muratori $^{3}$, Noit Inbar ${ }^{5}$, Tanya Gurevich ${ }^{5}$, Jeffrey Hausdorff ${ }^{5}$, Vincent Poile $^{6}$, Karen Marder ${ }^{7}$, Monica Busse ${ }^{6}$, Lori Quinn ${ }^{2}$

${ }^{1}$ Rehabilitation and Regenerative Medicine (Physical Therapy), G.H. Sergievsky Center, Columbia University, NY, USA; ${ }^{2}$ Movement Science, Teachers College, Columbia University, NY, USA; ${ }^{3}$ Physical Therapy, Wayne State University, Detroit, MI, USA; ${ }^{4}$ Physical Therapy, Stony Brook University, Stony Brook, NY, USA; ${ }^{5}$ Physical Therapy and Neurology, Tel Aviv University, Tel Aviv, Israel; ${ }^{6}$ Centre for Trials 
Research, Cardiff University, Cardiff, Wales, UK; ${ }^{7}$ Neurology, Taub Institute, GH Sergievsky Center, Columbia University, NY, USA

Introduction: People with Huntington disease (HD) have mobility impairments and increased risk for falls, which limit quality of life. We examined the spatial extent of mobility, characteristics and circumstances of falls in people with Manifest HD.

Methods: 50 people with HD $(53.4 \pm 11.8$ years $)$ and 28 controls $(52.3 \pm 10.6$ years $)$ were tested. Falls were examined with the History of Falls Questionnaire, and physical activity was quantified with the International Physical Activity questionnaire. Postural sway was measured with inertial sensors (APDM). We administered the Life-space questionnaire, a reliable and valid tool that measures the extent, frequency and assistance needed for spatial mobility patterns at home and community in a subset of participants $(n=30)$. Scores range from 0 (bed bound) to 120 (travel outside town). Clinical assessment included the UHDRS Total Motor Score, Symbol Digit Modality Test, and Functional Assessment. Pearson correlation and regression analyses were used to examine associations.

Results: Age, height and weight were similar across groups. Falls were reported by $58 \%$ of HD subjects, among whom $69 \%$ reported 2 or more falls. Common causes of falls included loss of balance (48\%), slips $(24 \%)$, trip/stumble $(20 \%)$ and quick movements $(20 \%)$. Indoor falls were most common $(58 \%)$ and environmental hazards contributed to $59 \%$ of falls. Falls were significantly associated with cognitive function $(\mathrm{r}=0.42, p=0.04)$. The mean Life-space score was $74.2 \pm 28.4$ (Range $8-120)$. $60 \%$ of HD subjects had scores indicating that their mobility was limited to their home and neighborhood. Life-space score was significantly correlated with postural sway $(-0.67, p=0.005)$ and UHDRS Functional Assessment $(0.49, p=0.01)$, but not with cognitive function or falls.

Conclusion: Cognitive impairments and environmental hazards contribute to falls, and postural instability contributes to falls and community mobility. Postural stability and environmental modifications may be important targets for intervention.

Lay Summary: Mobility and participation in community activity may prevent falls and contribute to quality of life in people with Huntington disease (HD). It is therefore important to examine patterns of community activity and factors associated with falls in people with HD.

\section{3}

\section{Microglia Activation in Basal Ganglia Is a Late Event in Huntington's Disease Pathophysiology}

Natalia P. Rocha ${ }^{1,2,3}$, Leigh B. Latham ${ }^{4}$, Odelin Charron ${ }^{5}$, Gabriela D. Colpo $^{3}$, Paolo Zanotti-Fregonara ${ }^{6}$, Leorah Freeman ${ }^{5}$, Erin Furr Stimming $^{2}$, Antonio L. Teixeira ${ }^{3}$

${ }^{1}$ Mitchell Center for Alzheimer's Disease and Related Brain Disorders, Department of Neurology, The University of Texas Health Science Center, Houston, TX, USA; ${ }^{2}$ HDSA Center of Excellence at University of Texas Health Science Center at Houston, Houston, TX, USA; ${ }^{3}$ Neuropsychiatry Program, Department of Psychiatry and Behavioral Sciences, McGovern Medical School, University of Texas, Houston, TX, USA; ${ }^{4}$ School of Medicine, University of Washington, Seattle, WA, USA; ${ }^{5}$ Department of Neurology, The University of Texas at Austin, Austin, TX, USA; ${ }^{6}$ Nantz National Alzheimer Center and Houston Methodist Research Neurological Institute, and Weill Cornell Medicine, Houston, TX, USA

Rationale: Previous studies have shown that inflammatory mechanisms are intrinsically associated with neurodegeneration. However, the role of both central nervous system and peripheral inflammation in HD is still poorly understood.
Objective: To investigate structural and functional changes in microglia in different stages of $\mathrm{HD}$.

Methods: 12 patients with a genetic diagnosis of HD [6 manifest patients $(50.8 \pm 11.5 \mathrm{y} ; 6 \mathrm{~F}), 6$ premanifest $\mathrm{HD}$ gene carriers $(38.2 \pm 7.2 \mathrm{y} ; 4 \mathrm{M} / 2 \mathrm{~F})]$, and 6 controls $(43.3 \pm 8.8 \mathrm{y} ; 5 \mathrm{~F} / 1 \mathrm{M})$ underwent a [11C]-ER176 PET scan and an MRI for anatomical localization. Segmentation of region of interest (ROIs) was performed and group differences in [11C]-ER176 binding (used to evaluate the extent of microglial activation) were assessed by the standardized uptake value ratio (SUVR), that is, the ratio of brain uptake in ROIs to that in the cerebellum. Blood samples were collected and monocytes were used to generate induced microglia-like cells (iMG). In vitro evaluation of the dynamic functions of microglia included a phagocytosis assay.

Results: Patients with HD present higher [11C]-ER176 SUVR in both left and right pallidi and putamens in comparison with controls $(p<0.05$; Kruskal-Wallis test followed by Dunn's test). No differences were observed when we compared premanifest HD with controls or with manifest HD. iMG from controls, premanifest HD and HD patients showed similar phagocytic capacity. However, cytokine production during phagocytosis was increased in iMG from patients with manifest $\mathrm{HD}$ in comparison with premanifest HD and controls.

Conclusions: Patients with manifest HD present increased microglia activation in basal ganglia structures that are known to be involved in HD pathophysiology. Premanifest HD gene carriers present SUVRs similar to controls, thus concluding that microglia activation occurs later in HD pathology. It is important to refine the understanding of the more specific immune/ inflammatory mechanisms that are involved in HD. This will allow the development of new therapeutic interventions to halt the progression of HD. Funding Information: Huntington's Disease Society of America (HDSA).

Lay Summary: Previous studies have shown that inflammatory/immune mechanisms are intrinsically associated with neurodegeneration. Herein, we investigated microglia activation (the brain immune cells) in different stages of HD. We found that patients with manifest HD (but not premanifest HD) present increased microglia activation in brain structures that are known to be involved in HD pathophysiology. It is important to refine the understanding of the more specific immune/inflammatory mechanisms that are involved in HD. This will allow the development of new therapeutic interventions to halt the progression of HD.

\section{4}

The Digital-HD Study: Smartphone-based Remote Testing to Assess Cognitive and Motor Symptoms in Huntington's Disease

Rosanna Tortelli ${ }^{1}$, Cedric Simillion ${ }^{2}$, Florian Lipsmeier ${ }^{2}$, Timothy Kilchenmann $^{2}$, Filipe Brogueira Rodrigues ${ }^{1}$, Lauren M Byrne ${ }^{1}$, Atieh Bamdadian $^{2}$, Christian Gossens ${ }^{2}$, Scott Schobel ${ }^{3}$, Michael Lindemann ${ }^{2}$, Edward J Wild ${ }^{1}$

${ }^{1}$ UCL Huntington's Disease Centre, UCL Queen Square Institute of Neurology, University College London, London, UK; ${ }^{2}$ Roche Pharmaceutical Research and Early Development, pRED Informatics, F. Hoffmann-La Roche Ltd., Basel, Switzerland; ${ }^{3} F$. Hoffmann-La Roche Ltd., Basel, Switzerland

The aim of this study is to evaluate the ability of smartphone-based remote testing to meaningfully quantify signs, symptoms and impairments among participants with premanifest Huntington's disease (HD) and manifest HD and compare them with healthy controls (HC).

A smartphone application comprising seven active tests (Symbol Digit Modalities Test [SDMT], Stroop Word Reading test [SWRT], Speeded tapping, Chorea, Balance, U-turn, 2-min walk) and continuous passive monitoring is deployed in an observational study (Digital-HD) of participants with premanifest HD, manifest HD and HC. Predefined active test measurements were aggregated over Weeks 5 and 6 post-screening to minimize practice effects. Group differences between HC, premanifest $\mathrm{HD}$ and manifest HD were analyzed using the Kruskall-Wallis test and 
subsequent pairwise Mann-Whitney tests. In an exploratory analysis, a broader set of features was analyzed for differences between $\mathrm{HC}$ and participants with premanifest HD.

Recruitment for Digital-HD is ongoing. Preliminary data were analyzed from 18 participants with premanifest HD, 15 with manifest HD and 19 HC. Predefined digital tests generally had close correspondence with their commonly used in-clinic counterparts and these significantly differed between $\mathrm{HC}$ or the premanifest HD group and the manifest HD group ( $p=$ $0.01-0.05$ for U-Turn speed and 2-min walk step frequency variability, $p<$ 0.01 for all other tests); however, no differences were observed in these tasks between $\mathrm{HC}$ and the premanifest HD group. The exploratory analysis identified several features with high face validity that significantly differed between $\mathrm{HC}$ and the premanifest group (e.g. tapping regularity in the speeded tapping test and drawing speed and accuracy in the Draw-a-shape test). This preliminary analysis suggests that the digital platform can robustly measure established features of manifest $\mathrm{HD}$, and affords the opportunity for innovative measures that may have great value in assessing premanifest and prodromal phases of HD.

Funding Information: Study funded by F. Hoffmann-La Roche Ltd.

Lay Summary: Digital-HD is a study to compare smartphone-based assessments in Huntington's disease (HD). Healthy controls and people with premanifest HD were found to perform differently from people with manifest HD in several tests, implying that this technology has the potential to shed light on disease symptoms and progression during the early stages of HD.

75

Cerebral Blood Flow and Affect Recognition in Early Premanifest and Manifest Huntington's Disease

M. Agustina Rossetti, Erin Furr Stimming, Natalia Pessoa Rocha McGovern Medical School at UTHealth Houston, Department of Neurology (all authors)

Objective: Emotion recognition deficits have been well studied in Huntington's disease (HD). The objective of this study was to examine the association between deficits in affect recognition and cerebral blood flow in early premanifest and manifest HD.

Methods: Seven control, seven early premanifest (11.67 \pm 7.09 years before the predicted clinical diagnosis), and seven manifest participants completed neuropsychological measures, including an affect naming scale. As part of a larger HDSA funded study, they underwent brain magnetic resonance imaging (MRI) at $3 \mathrm{~T}$ with pseudo-continuous arterial spin labeling (pCASL) to quantify cerebral blood flow (CBF), and 3D-MPRAGE for volumetric segmentation. pCASL images were analyzed with the FMRIB Software Library (FSL) and FreeSurfer v5.3.0. Region of interest (ROI) analyses were performed to analyze the correlation between $\mathrm{CBF}$ in specific brain regions and affect naming scores (Spearman correlations).

Results: No statistically significant differences among the three groups were observed in age, education, or gender. CAG length did not statistically differ between the manifest $(44.67 \pm 2.42)$ and premanifest $(42.67 \pm$ 1.86) participants $(p=0.140)$. Compared to healthy controls and early premanifest individuals, manifest participants had significant difficulties with affect naming with the highest number of errors made on items assessing perception of negative emotions. Among manifest HD, affect naming (total correct responses) correlated with $\mathrm{CBF}$ in left pallidum (rho $=-0.883, p=0.020)$, left amygdala (rho $=0.971, p=0.001)$, and left insula ( $\mathrm{rho}=0.883, p=0.020$ ). These correlations were not found among controls or the early premanifest HD group.

Conclusion: Early premanifest and healthy controls do not differ in their ability to recognize emotions from facial stimuli. There were no correlations between affect naming and regional $\mathrm{CBF}$ in the early premanifest group. Among individuals with manifest HD, there were strong associations among emotion recognition and CBF in the left insula and amygdala, areas well established in the neurobiology of emotional perception. Future studies examining these associations in prodromal HD are warranted to help inform behavioral interventions if and when a disease modifying therapy is approved.

Lay Summary: This pilot study examines affect recognition and cerebral perfusion in early premanifest and manifest HD patients. This research may help elucidate early changes in blood flow in the disease spectrum and help inform when appropriate interventions that target emotion recognition training may be implemented.

\section{6}

\section{Sleep Disturbance in Huntington's Disease - Observational Study}

Kasia Gustaw Rothenberg, Lyndsey Sandy, Anwar Ahmed

Cleveland Clinic, Cleveland, OH, USA (all authors)

Objective: Both mood disorders as well as sleep disturbances are a prominent feature of Huntington's disease, and substantially impair patients' quality of life (Bellosta Diago et al. 2017, Simpson et al. 2016). Similarly, both in Huntington's disease (HD) as in depression, sleep disturbance becomes one of the dominant pathognomonic features (Morton et al. 2014). Moreover, sleep problems may be the first sign of the onset of a major depressive episode. Some studies involving HD patients suggest that a deficit contributing to disordered sleep is reduced suprachiasmatic nucleus output. The mechanism underlying this deficit is not yet known, but mitochondrial dysfunction and oxidative stress are likely involved (Pallier et al. 2007, Van Wamelen et al. 2013).

This raises the possibility that the disordered sleep and circadian function experienced by HD patients may be an integral part of the disease.

Aim: To delineate the nature of sleep disturbances and identify correlates of sleep impairment in the context of depression in HD patients.

Subjects:

Demographics, genetic, mood and sleep parameters were analyzed in:

- $\quad 37$ Patients (10 M and $27 \mathrm{~F}$ ) with symptomatic Huntington's disease

- Mean age 51 (range 32 to 79 ; median 51)

- Mean numbers of CAG repeats 44 (range 38-62; median 43)

- All diagnosed and treated in HDSA Center of Excellence at Cleveland Clinic (assessment between February 2017 and July 2018).

\section{Methods:}

- Clinical questionnaires for sleep and psychiatric assessment for depression were used.

- Sleep-wake disorders were categorized according to DSM 5 criteria.

- Depression was quantitatively assessed by PHQ 9 (maximum score 27).

- Study protocol was approved by The Cleveland Clinic IRB (\# 18-1040).

- Descriptive statistics and correlates were calculated with Statistica.

Results:

- All examined Patient but four report history of at least one episodes of major depressive disorder $(80 \%)$.

- Of thought all we actively treated for depression (55\% SSRI, $26 \%$ SNRI, $18 \%$ the other groups of antidepressants, $60 \%$ of Patients were on two on more agents).

- At the time of evaluation $88 \%$ of patient were in at least partial remission.

- According to PHQ9 (mean score 10.2 range 0-22, median 8) patient presented only with minimal (PHQ1-4) or mild depression (PHQ59) at the time of evaluation. 
- $\quad$ Sleep impairment was prevalent in HD Patients and was reported by almost all examined but two $(94.5 \%)$.

- Circadian rhythm sleep-wake disorders with delayed sleep phase type dominated in the group $(30 / 37,81 \%)$

- $\quad$ Followed by insomnia $(26 / 37 ; 70 \%)$ and

- Hypersomnia $(7 / 37 ; 18 \%)$.

- More than $60 \%$ of Patients suffers from two or more sleep disorders

- Only $13 \%$ of Patients however reported the most characteristic for depression early morning awakenings.

- Correlations:

- History of depression correlated with sleep disturbance $(r=+0.76)$ but not with number of CAG repeats $(r=+0.17)$.

- Treatment and remission of depression did not correspond with improvement in the sleep disorders $(r=+0.2)$.

- $\quad$ Sleep impairment seem to persist beyond any form of treatment for depression in (32/37); $86 \%$ of Patients.

Conclusions: HD is primarily accompanied by circadian rhythm sleep disturbances, delayed sleep phase, which are associated with depression but does not respond to depression management. This raises the possibility that the disordered sleep and circadian function experienced by HD patients may be an integral part of the disease independent from mood disorder. If these hypotheses are correct, we should focus on treating circadian misalignment and sleep disruptions early in disease progression parallel to addressing depression.

Lay Summary: HD is primarily accompanied by circadian rhythm sleep disturbances, delayed sleep phase, which are associated with depression but does not respond to depression management. This raises the possibility that the disordered sleep and circadian function experienced by HD patients may be an integral part of the disease independent from mood disorder. If these hypotheses are correct, we should focus on treating circadian misalignment and sleep disruptions early in disease progression parallel to addressing depression.

77

Abnormal Development of Cerebellar-Striatal Circuitry in Huntington's Disease

Jordan L. Schultz, Alexander V. Tereshchenko, Joel E. Bruss, Vincent A. Magnotta, Eric A. Epping, Peg C. Nopoulos

The University of Iowa, Iowa City, IA, USA(all authors)

Objective: To investigate functional connectivity differences over time of the striatum and the cerebellum between pre-symptomatic patients with the HD gene expansion (GE) and patients with a family history of HD but without the gene-expansion (GNE).

Methods: This study utilized resting-state, functional MRI (rsfcMRI) data from participants enrolled in the Kids-HD study who were 6 to 18 years old. Participants were divided into the GE (CAG 36-59) and GNE (CAG <36) groups. Seed to seed correlations were calculated between four regions that provide input signals to the anterior cerebellum (aCB): 1) Dorsolateral Putamen (dPU);2) Globus Pallidus Externa (GPE); 3) Subthalamus Nucleus (STN), and 4) Pontine Nuclei (PN) and two regions that represented output from the cerebellum, the Dentate Nucleus (DN) to the: 1) Ventrolateral Thalamus (VL) and 2) dPU. Linear mixed effects regression models evaluated whether the trajectory of development of these connections over time differed between groups.

Results: Four of the six striatal-cerebellum correlations showed significantly different trajectories between groups. All showed a pattern where in the early age ranges (6-12 years), there was hyperconnectivity in the GE compared to the GNE with those trajectories showing linear decline in the latter half of the age range.

Discussion: These results parallel previous findings showing striatal hypertrophy in GE expanded children as early as age 6. These findings support the notion of developmentally higher connectivity between the striatum and cerebellum early in the life of the child with the HD gene expansion, possibly setting the stage for cerebellar compensatory mechanisms.

Lay Summary: This study demonstrates that children who have tested positive for the HD gene expansion have significantly difference trajectories over time of functional connectivity between the striatum and cerebellum compared to patients without the gene expansion. These findings are vitally important to enhancing our understanding of how the presence of mutant huntingtin affects neurodevelopment. It also provides further evidence that the cerebellum may play a compensatory role in HD and may be a viable therapeutic target for HD.

\section{8}

Higher CAG Repeats Below Disease Threshold Confers Cognitive Advantage

Jordan L. Schultz ${ }^{1}$, Carsten $\mathrm{Saft}^{2}$, Peg C. Nopoulos ${ }^{1}$

${ }^{1}$ University of Iowa, Iowa City, IA, USA; ${ }^{2}$ Huntington Center NRW, Ruhr-University Bochum, St Josef-Hospital, Bochum, Germany

Objective: To investigate the relationship between CAG repeat length in patients with and without Huntington's Disease (HD) and cognitive performance.

Methods: We analyzed the fourth data cut (released December 2018 ) of the Enroll-HD dataset. Young adults ( $\leq 25$ years) without a history of depression, anxiety, or apathy were included in the analysis. We included participants with and without the geneexpansion $(\mathrm{CAG} \geq 36$ ) for $\mathrm{HD}$. The participants with the geneexpansion had to have a Total Functional Capacity Score of 13, a diagnostic confidence level of less than four, and total motor score of $<20$, and be more than 20 years from their predicted motor onset. We performed linear mixed effects regression analyses to investigate the non-linear (quadratic) relationship between CAG repeat length (15 to 46) and various cognitive measures. The models included a random effect per subject and adjustments for age, sex, and educational attainment.

Results: There were significant relationships between CAG and Symbol Digit Modalities Test $(p=0.0005)$, Stroop Color Naming Test $(p=0.004)$, and part A of the Trails Making Test $(p=0.035)$. For all of these findings, performance improved below the disease threshold $(\mathrm{CAG}<36)$ and peaked at a $\mathrm{CAG}$ repeat length of approximately 40 .

Discussion: These results provide further evidence that an increasing number of $\mathrm{CAG}$ repeats in the huntingtin protein below disease threshold confer a potential evolutionary advantage in cognitive performance. The advantage begins to decrease at repeat numbers above approximately 40 . These findings highlight the potential importance of the wild-type huntingtin protein.

Lay Summary: The normal function of the huntingtin protein is still unclear, but previous studies have demonstrated that increased CAG repeat length below disease threshold $(<36)$ confers cognitive and intellectual advantages. This study aimed to investigate the relationship between CAG repeat length and cognitive function among healthy controls and patients with HD in a large patient population. This information is critically important to the HD community as it may provide evidence for an evolutionary advantage of increased CAG repeat length in healthy controls, which may increase our understanding of the function of the huntingtin protein. 
79

Preliminary Data from Longitudinal Study of Neuropsychiatric Symptoms in Huntington's Disease (LATNS-HD)

Sellers J, Isaacs DA, Claassen DO

Vanderbilt University Medical Center, Nashville, TN, USA (all authors)

Objective: 1) To determine the level of agreement between psychiatry and neurology assessment in detection and quantification of neuropsychiatric symptoms in HD and 2) To present preliminary data on the effects of neuropsychiatric symptoms on quality of life in HD.

Background: Neuropsychiatric symptoms are common in HD, though they are often underrecognized and under-treated. Currently, assessment by a psychiatrist is the gold-standard for detection of these symptoms, but many HD providers do not have access to specialized psychiatry resources. Here we present preliminary data from an ongoing, longitudinal study of neuropsychiatric symptoms in HD. Our goal for this analysis was to assess the amount of agreement between psychiatry and neurology clinic assessment in identification and rating of neuropsychiatric symptoms in HD. We also present data on the distribution of symptoms and preliminary effect sizes for correlation of these symptoms with quality of life.

Methods: Fifty HD patients and their caregivers were recruited for Phase A of this longitudinal study. Each patient completed a battery of scales to assess neuropsychiatric symptoms, patient-reported outcomes, quality of life, and other clinical characteristics. A psychiatrist familiar with HD also evaluated each patient, then completed a Likert scale to rate presence and severity of several neuropsychiatric symptoms including depression, anxiety, apathy, irritability/aggressive behavior, obsessive or preservative behavior, and delusions or hallucinations. We plan to use ROC curves to evaluate the level of agreement between the psychiatrist's assessment and our battery of scales used in neurology clinic. We will also evaluate the distribution of neuropsychiatric symptoms reported at baseline. Canonical correlations will be used to detect associations between neuropsychiatric symptoms and quality of life outcomes.

Results of the above analyses will be presented and implications for research and clinical practice will be discussed.

Lay Summary: Neuropsychiatric symptoms are common in HD, though they are often underrecognized and under-treated. Currently, assessment by a psychiatrist is the gold-standard for detection of these symptoms, but many HD providers do not have access to specialized psychiatry resources. In this study we assessed the amount of agreement between psychiatry and neurology clinic assessment of neuropsychiatric symptoms in HD to inform best practice for future research and clinical practice.

\section{0}

Central Cognitive Processing Speed Is an Early Marker of Huntington's Disease Onset

Chase Snell ${ }^{1}$, McKenna E. Williams ${ }^{2}$, Sameer Aboufadel ${ }^{1}$, Paul E. Gilbert $^{2,3}$, Jody Corey-Bloom ${ }^{1,3}$

${ }^{1}$ Department of Neurosciences, University of California, San Diego, La Jolla, CA, USA; ${ }^{2}$ Department of Psychology, San Diego State University, San Diego, CA, USA; ${ }^{3}$ San Diego State University/University of California San Diego Joint Doctoral Program in Clinical Psychology, San Diego/La Jolla, CA

Objective: The objective of the current study was to examine central cognitive processing speed, as measured using the Computerized Test of Information Processing (CTIP), as an early marker of Huntington's disease (HD) onset.

Background: Several studies have suggested that cognitive processing speed may be particularly useful for assessing early cognitive change in individuals with premanifest HD (Pre-HD); however, current measures lack the ability to control for the effects of motor dysfunction that is common in HD. The CTIP is a rapidly administered and useful computerized tool that allows for examination of central cognitive processing speed by using motor-corrected scores to account for motor dysfunction. Methods: The CTIP, along with a standardized neuropsychological battery, was administered to 100 gene carriers and 55 healthy adults (HA). Gene carriers included 1) Pre-HD, $n=33 ; 2$ ) transitional HD (T-HD, i.e., individuals close to disease onset or with very mild $\mathrm{HD}, n=23$; and 3) mild-moderate $\mathrm{HD}, n=46$.

Results: The HD group performed significantly slower than the HA, Pre$\mathrm{HD}$, and T-HD groups on both motor-corrected subtests $(\mathrm{ps}<.05)$. Additionally, the T-HD group performed significantly slower than the HA group on both motor-corrected subtests (ps <.05). Importantly, effect sizes associated with significant group differences between the T-HD and HA groups on motor-corrected CTIP subtests $(\mathrm{d}=.73$ and .84$)$ were similar to effect sizes associated with group differences on the Symbol Digit Modalities Test $(\mathrm{d}=.82)$, a standardized measure of processing speed that does not correct for motor dysfunction, and other traditional cognitive assessments (MoCA d $=.75$; MMSE $\mathrm{d}=.84$ ).

Conclusions: Our results suggest that the CTIP may be a useful marker of deficits in central cognitive processing speed in individuals close to HD onset. Lay Summary: The purpose of this study was to see if central processing speed could be an early marker of HD onset. We found that HD subjects and, more importantly, individuals transitioning to $\mathrm{HD}$, showed slowed central cognitive processing compared to healthy adults. This suggests that central processing speed may be a useful and early marker of progression to HD.

\section{1}

\section{Decoding CSF Mutant Huntingtin}

Nicholas S. Caron ${ }^{1}$, Ethan D. Smith ${ }^{2}$, Christopher Yanick ${ }^{2}$, Yuanyun $\mathrm{Xie}^{2}$, Stephen E.P. Smith ${ }^{3}$, Ji Joon Song ${ }^{4}$, Ihnsik Seong ${ }^{5}$, Blair R. Leavitt ${ }^{1}$, Michael R. Hayden ${ }^{1}$, Amber L. Southwell ${ }^{2}$

${ }^{1}$ Center for Molecular Medicine and Therapeutics, University of British Columbia, Vancouver, Canada; ${ }^{2}$ Burnett School of Biomedical Sciences, University of Central Florida, Orlando, FL, USA; ${ }^{3}$ Seattle Children's Research Institute, University of Washington, Seattle, WA, USA; ${ }^{4}$ Department of Biological Sciences, KAIST, Daejeon, Korea; ${ }^{5}$ Department of Neurology, Massachusetts General Hospital, Boston, MA, USA

The first huntingtin (HTT) lowering safety trial recently completed, demonstrating dose-dependent reduction of cerebrospinal fluid (CSF) mutant (mt) HTT. However, we do not know where CSF mtHTT comes from or how it enters CSF, which confounds interpretation of treatment-induced changes. We are using immunoprecipitation and flow cytometry (IPFCM) mtHTT detection to query what this data tells us about therapeutic activity in the brain.

The source of CSF mtHTT is unknown. Some therapeutics, such as ASOs, may be most active in regions in contact with the CSF. If these regions are the major sources of CSF mtHTT protein, then CSF-based predictions of HTT lowering in deeper structures may not be straightforward. Conversely, if regional contributions to CSF mtHTT are similar, then changes in CSF mtHTT level could be used to more accurately infer changes in basal ganglia mtHTT. Thus, we are interrogating the source(s) of CSF mtHTT protein using genetic approaches and ectopic delivery of mtHTT.

Additionally, we do not know how mtHTT enters CSF. Acute brain injury causes a transient increase in CSF mtHTT, suggesting that mtHTT is released from dying neurons. Thus, any neuroprotective therapy would be expected to reduce CSF mtHTT, and HTT lowering treatment-induced changes may represent a combination of target engagement and neuroprotection. However, inhibiting HTT secretion reduces CSF mtHTT protein concentration, suggesting that there may be both passive and active clearance mechanisms involved. If active clearance is the primary 
mechanism of mtHTT release, then treatment-induced changes may mostly represent HTT lowering. To investigate the mechanism(s) of mtHTT release to CSF, we are ectopically delivering intra and extracellular mtHTT with or without inhibitors of secretion and glymphatic clearance in the presence or absence of neuronal insult.

Delineating the source and mechanism(s) of entry of CSF mtHTT protein will greatly enhance interpretation of this promising HD biomarker.

Lay Summary: The first huntingtin lowering clinical trial recently demonstrated dose-dependent reduction of mutant huntingtin in the spinal fluid of treated Huntington disease patients. We are investigating where huntingtin protein in spinal fluid comes from and how it gets there to better understand what this exciting new data means about the brain.

\section{2}

A Novel Repeat-Primed PCR Assay to Detect the Full Range of Trinucleotide CAG Repeats Including Pediatric Huntington Disease Associated Alleles

A. De Luca ${ }^{1}$, F. Consoli ${ }^{1}$, A. Morella ${ }^{1}$, A. Onori ${ }^{1}$, M. D Asdia ${ }^{1}$, S. Fanelli $^{2}$, B. D Alessio ${ }^{3}$, F. Squitieri ${ }^{2,3}$

${ }^{1}$ UOS Diagnosi Genetica Molecolare, Fondazione IRCCS Casa Sollievo della Sofferenza, San Giovanni Rotondo and CSS-Mendel, Rome, Italy; ${ }^{2}$ UOI Huntington and Rare Diseases Unit, Fondazione IRCCS Casa Sollievo della Sofferenza, San Giovanni Rotondo and CSS-Mendel, Rome, Italy; ${ }^{3}$ Fondazione Lega Italiana Ricerca Huntington (LIRH), Rome, Italy

Introduction: Pediatric Huntington disease (PHD) is a rare condition associated with unusually large HTT CAG repeat size, usually of 60 CAG repeats or more. It affects adolescent patients before $18 \mathrm{yrs}$. of age, and very rarely young children before 10 yrs. of age, with particularly severe presentation and course. In these patients, accurate determination of the CAG repeat number is recommended to confirm the diagnosis. Here we evaluated the reliability of a novel tripled repeat primed PCR-based method (AmplideX PCR/CE HTT Kit) for accurate estimation of HTT CAG repeats in PHD.

Materials and Methods: Study cohort included a total of 66 DNA samples, comprising: i) a first reference set of 14 cell-line samples (Coriell) with associated genotype data; ii) a second reference set of 5 samples previously genotyped by multiple laboratories, including two cases with alleles larger than $60 \mathrm{CAG}$ repeats; and iii) a set of 47 samples previously genotyped in our laboratory by flurescent repeat-flanking PCR encompassing the full range of trinucleotide CAG repeats in HTT gene, but enriched for alleles larger than 60 CAG repeats.

Results: All samples showed full concordance with the previously verified allele sizes. Identical repeat size or sizing errors within \pm 1 CAG were obtained for alleles $\leq 42$ CAG repeats, whereas sizing errors within \pm 3 CAG were obtained for alleles $>43$ CAG repeats, conforming to established guidelines. The assay was able to accurately size 21 samples with very large alleles comprised between 60 and $100 \mathrm{CAG}$ repeats, and to detect two exceptionally large alleles with more than $200 \mathrm{CAG}$ repeats.

Conclusions: This method provides a rapid, sensitive and reliable strategy to accurately genotype the HTT CAG repeat stretch, and extends the detection limit of large expanded alleles to over 200 CAG repeats, thus providing a comprehensive molecular diagnostic evaluation of all Huntington disease samples, including those pediatric forms carrying extremely large, hard to detect, alleles.

Lay Summary: The method we describe provides a rapid, sensitive and reliable strategy to accurately genotype the HTT CAG repeat stretch, and extends the detection limit of large expanded alleles to over $200 \mathrm{CAG}$ repeats, thus providing a comprehensive molecular diagnostic evaluation of all Huntington disease samples, including those pediatric forms carrying extremely large, hard to detect, alleles.
83

Exploring Executive Functions in Premanifest and Prodromal Huntington's Disease Subjects

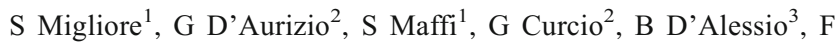
Squitieri ${ }^{1,3}$

${ }^{1}$ UOI Huntington and Rare Diseases Unit, Fondazione IRCCS Casa Sollievo della Sofferenza Hospital, San Giovanni Rotondo and CSSMendel, Rome, Italy; ${ }^{2}$ Department of Biotechnological and Applied Clinical Sciences, University of L'Aquila, L'Aquila, Italy; ${ }^{3}$ Fondazione Lega Italiana Ricerca Huntington (LIRH), Rome, Italy

Introduction: Huntington's Disease (HD) cognitive dysfunction may occur before unequivocal motor signs become apparent. Indeed, composite measures to track presymptomatic life stage include both cognitive and motor assays. We tested executive functions in premanifest and prodromal HD subjects by different assays: 1) task-switching; 2) risky decisionmaking process by means Game of Dice Task (GDT).

Methods: In study 1 we recruited 15 preHD1 subjects with a TMS $\leq 4$ (far from onset), 15 preHD2 subjects with a $5 \leq \mathrm{TMS} \leq 9$ and 18 age-sexeducation matched Healthy Controls (HC). In study 2 we recruited 30 preHD subjects $(\mathrm{TMS}<10)$ and 21 age-gender-education matched $\mathrm{HC}$. Results: Study 1: All preHD subjects had worse performance than $\mathrm{HC}$ in the switch and repetition trials, as indicated by increased switch-cost and reaction times. In particular, preHD2 showed impaired switching abilities with reaction times slower than preHD1 and HC. Study 2: Decision and feedback times were longer in preHD than in $\mathrm{HC}$ in both disadvantageous and advantageous choices. Moreover, preHD provided a greater number of "safe" strategies, taken with longer decision-making time than HC, showing a reduced propensity to risk.

Conclusions: Our studies highlighted early task-switching impairment and economic decision-making slowness with a reduced propensity to risk since HD was still at a pre-manifest stage. Such abnormalities worsen when preHD subjects start to show subtle motor manifestations, still nonspecific and insufficient to define the clinical diagnosis of HD $(\mathrm{DCL}<4)$. Such dysfunctions might contribute to delineate an early behavioral and cognitive profile in premanifest stage of the disease. Moreover, considering that such abilities have obvious implications for activities of daily living, early cognitive rehabilitation addressing such deficits might be useful in this stage of the disease.

Lay Summary: Executive functions dysfunction contribute to delineate an early behavioral and cognitive profile in presymptomatic life stage and may contribute to highlight new potential clinical markers to further update composite measures and new potential targets for therapies.

\section{4}

Assessment of Post-dural Puncture Headache Frequency in a Huntington's Disease Clinical Research Population

Ragini Srinivasan, Bhavpreet Dham, Mark Guttman Centre for Movement Disorders, Toronto, Ontario, Canada (all authors)

Introduction: Lumbar puncture (LP) for CSF sampling and intrathecal bolus (ITB) drug administration are being performed more frequently in Huntington's disease (HD) research. Post-dural puncture headache $(\mathrm{PDPH})$ has been reported in the literature as a complication affecting up to $35 \%$ of subjects. As predictors of PDPH in the HD population are not established, we reviewed the rate and risk factors for PDPH in HD patients at our centre.

Methods: A retrospective review of LPs performed for research related CSF sampling and ITB research drug administration over the last 30 months was performed. All LPs were first attempted at the L3-4 interspace with either $22 \mathrm{G}$ or $24 \mathrm{G}$ Whitacre atraumatic needles. LPs performed on the same patient at various time points were considered independent procedures. 
Results: A total of 184 procedures were performed in 78 individuals, of which 178 were successful in collecting CSF. The median age of our cohort was 50 years (range 22-73) of whom $41 \%$ were male. Symptomatic HD subjects accounted for 116 LPs (65\%), whereas presymptomatic HD subjects and control subjects accounted for $34(19 \%)$ and $28(16 \%)$ LPs, respectively. There were 94/178 (52.8\%) LPs performed with the $22 \mathrm{G}$ needle in 63 patients and 84/178 (47.2\%) LPs performed with the $24 \mathrm{G}$ needle in 15 patients.

PDHP occurred with 10/94 (10.6\%) LPs that were performed using 22G needle whereas none of the 84 LPs using $24 \mathrm{G}$ were associated with PDHP. This difference across the two needle sizes was statistically significant $(p=0.0017)$. One patient with PDPH required an epidural blood patch.

Conclusion: This retrospective chart review showed PDPH of $10.6 \%$ in the $22 \mathrm{G}$ needle group compared to $0 \%$ in the $24 \mathrm{G}$ group. Based on these observations, we recommend all studies utilize the $24 \mathrm{G}$ atraumatic LP needles for HD CSF and ITB studies.

Lay Summary: Lumbar punctures (LPs) are being performed more in Huntington's Disease research, and a common complication is a severe headache that improves when lying flat, called a postdural puncture headache. We reviewed the LPs done at our site to see if using a smaller spinal needle led to fewer headaches. Postdural puncture headaches happened in $10.6 \%$ of LPs with a standard $(22 \mathrm{G})$ needle and did not occur at all with the smaller (24G) needle. We suggest that HD studies use the smaller $24 \mathrm{G}$ needle for LPs to reduce the risk of headaches.

\section{5}

Adipose-derived Mesenchymal Stem Cells Ameliorate Neuroinflammation and Promote Neurogenesis in a Transgenic Mouse Model of Huntington's Disease

Amit K. Srivastava ${ }^{1}$, Jose F. Moruno-Manchon ${ }^{2}$, Karthik S. Prabhakara ${ }^{1}$, Akshita Kumar $^{1}$, Naama E. Toledano Furman ${ }^{1}$, Alex Villarreal ${ }^{1}$, Gabriela D. Colpo ${ }^{3}$, Andrey S. Tsvetkov ${ }^{2}$, Antonio L. Teixeira Jr. ${ }^{3}$, Erin FurrStimming ${ }^{4}$, Scott D. Olson ${ }^{1}$

${ }^{1}$ Department of Pediatric Surgery, University of Texas Health Science Center at Houston, McGovern Medical School, Houston, TX, USA; ${ }^{2}$ Department of Neurobiology and Anatomy, The University of Texas, Houston Medical School, Houston, TX, USA; ${ }^{3}$ Department of Psychiatry and Behavioral Sciences The University of Texas, Houston Medical School, Houston, TX, USA; ${ }^{4}$ Department of Neurology, The University of Texas Health Science Center at Houston, Houston, TX, USA

Huntington's disease (HD) is a genetically dominant neurodegenerative disorder characterized by progressive motor, cognitive, and psychiatric disturbances associated with neuronal dysfunction and atrophy of the striatum and other brain regions. To date, there are no effective disease modifying treatments approved for HD. Because restorative therapies targeting the underlying cause of neuronal dysfunction do not exist, therapies with anti-inflammatory, neuroprotective and regenerative potential hold great promise. Neuroinflammation is an early pathological feature of HD. Previous studies reported Ibal and GFAP immunoreactivities in the brains of presymptomatic carriers and an increase with disease progression. Mesenchymal stem cells (MSCs) have demonstrated significant therapeutic effects in various neuroinflammatory diseases. In this study, we examined the therapeutic effect of a commercial preparation of adipose-derived MSCs (HB-AdMSCs) on neuroinflammation and neurogenesis in a transgenic HD mouse model. The cells were obtained from Hope Biosciences (Sugar Land, TX) and intravenously infused twice, first at 7 weeks of age and then at 10 weeks of age of B6CBAR6/2 transgenic HD or wildtype mice. No immunosuppressive drug was given to any animal. The animals were perfused 14 days after the second cell injection to harvest the brains and perform immunohistochemical assays. We observed that HB-AdMSCs were able to significantly reduce astrocyte reactivity in the cortical region of the HD brains compared to controls. The cells also significantly reduced microglial reactivity and induced neurogenesis in the striatum. We conclude that administration of HB-AdMSCs could ameliorate the inflammatory microenvironment in the brain to support survival of damaged neurons and improve neurogenesis in experimental HD.

Lay Summary: Adipose-derived mesenchymal stem cells therapy could be a good therapeutic strategy for HD.

\section{6}

\section{Patterns of Genetic Testing in Huntington's Disease}

Karina Raimundo ${ }^{1}$, Ruoding $\operatorname{Tan}^{1}$, Tu My To ${ }^{1}$, Jonathan de Courcy ${ }^{2}$, Umang Ondhia, ${ }^{3}$ Huu Phuc Nguyen ${ }^{4}$, Martha Nance ${ }^{5}$

${ }^{1}$ Genentech Inc., South San Francisco, CA, USA; ${ }^{2}$ Adelphi Real World, Bollington, Cheshire, UK; ${ }^{3}$ F. Hoffmann-La Roche Ltd., Basel, Switzerland; ${ }^{4}$ Department of Human Genetics, Ruhr University Bochum, Bochum, Germany; ${ }^{5}$ Struthers Parkinson's Centre, Golden Valley, MN, USA

Huntington's disease (HD) is a genetic disease caused by a CAG trinucleotide repeat expansion in the huntingtin gene. Genetic testing can identify the presence of the CAG repeat expansion before disease manifestation. Here, we describe patterns of genetic testing in five countries.

This is a retrospective analysis of data from the Adelphi HD Disease Specific Programme - a cross-sectional survey of neurologists and patients conducted between July-October 2017 in France, Germany, Italy, the UK and the US. We describe physician-reported rates of, and reasons for, predictive and confirmatory genetic testing. Only responses with consistent and non-conflicting reporting of family history of HD were included.

This analysis included physician-reported data on 985 manifest patients. Overall, $46 \%$ of patients were not aware of family history of HD. Most patients $(70 \%)$ received, at some point, genetic testing confirming HD. Predictive testing was reported in only $23 \%$ of patients who were later clinically diagnosed with HD. Among all reasons reported for undergoing predictive testing, the most common reasons were: desire to know (64\%) and awareness of other family members being diagnosed (23\%). For the $73 \%$ of patients who chose not to undergo predictive testing, the most common reasons were: unaware of family history (48\%), rather not know $(25 \%)$, and no treatment for disease available $(12 \%)$.

Currently not all patients with HD receive confirmatory genetic testing. Rates of testing prior to clinical diagnosis are even lower. Among the reasons not to be tested is the lack of treatments available. In the event therapies are proven to change the course of disease, these patterns may change, potentially leading to an increase in rates of confirmatory testing. Nonetheless, the decision to receive predictive testing is personal and can be influenced by multiple factors.

Funding Information: Study sponsored by Genentech Inc. and F. Hoffmann-La Roche Ltd.

Lay Summary: The authors of this study analyzed data from a survey of physicians and patients with manifest Huntington's disease (HD) to understand how genetic testing is used in HD and what motivates a person to take the genetic test. Most patients (70\%) received a genetic test to confirm their diagnosis of HD, however only $23 \%$ of patients received the test before symptoms of HD developed. Wanting to confirm a diagnosis of HD was the most popular reason for taking a genetic test (64\% of 160 patients surveyed), while not being aware of a family history of HD was the most common reason for not taking a genetic test ( $48 \%$ of 502 patients surveyed).

\section{7}

Sleep Complaints Are Associated with Anxiety Symptoms and Poorer Quality of Life in HD

Will K. Tanigaki ${ }^{1}$, Maria A. Rossetti ${ }^{1,2}$, Sudha Tallavajhula ${ }^{2}$, Natalia P. Rocha $^{1,2}$, Erin Furr Stimming ${ }^{1,2}$ 
${ }^{1}$ HDSA Center of Excellence at University of Texas Health Science Center at Houston, Houston, TX, USA; ${ }^{2}$ Department of Neurology, The University of Texas Health Science Center at Houston, Houston, TX, USA

Background: Notwithstanding the high prevalence and the availability of clinical tools, sleep disorders are still not routinely evaluated in patients with HD. Sleep disturbances can seriously affect the quality of life of the patient, caregiver and family. In addition, sleep dysfunction can result in cognitive impairment and mood disorders. Therefore, the current study was designed to investigate sleep quality and its association with clinical symptoms in HD.

Methods: Fifteen patients with HD $(51.15 \pm 12.66$ years, $10 \mathrm{~F} / 5 \mathrm{M})$ completed a series of self-reported questionnaires about sleep quality and pattern [Pittsburgh Sleep Quality Index (PSQI)], daytime sleepiness [Epworth Sleepiness Scale (ESS)], cognitive function (NeuroQOLv2.0 Cognition Function), quality of life [HD Quality of Life Questionnaire (HDQoL)] and depressive and anxious symptoms [Hospital Anxiety and Depression Scale (HADS)]. Spearman correlation analyses were performed to ascertain the association between sleep quality and severity of self-perceived clinical symptoms.

Results: The primary sleep complaints reported by the patients were related to waking up in the middle of the night or early in the morning; and increased sleep latency. Nine out of the 15 patients $(60 \%)$ reported the use of sleep medications at least once a week. We did not find any significant association between sleep quality and self-perceived cognitive function or depressive symptoms. Worse sleep quality was associated with greater severity of anxiety symptoms, as observed by the significant correlations between HADS-anxiety score and PSQI global score (rho = $0.561, p=0.046$ ) and PSQI components: subjective sleep quality (rho = $0.577, p=0.039$ ); sleep latency ( $r h o=0.675, p=0.011)$; sleep duration $($ rho $=0.586, p=0.035)$; sleep disturbances $(\mathrm{rho}=0.733, p=0.004)$. Importantly, greater severity of sleep latency and sleep disturbances were associated with worse quality of life (rho $=0.673, p=0.012$; and rho $=$ $0.679, p=0.011$, respectively)

Conclusions: In our experience, sleep dysfunction in patients with HD is associated with increased anxiety and worse quality of life. Identifying sleep dysfunction in HD is essential as this could significantly impact the optimal management of individuals with HD.

Lay Summary: Fifteen HD patients completed a series of questionnaires about sleep quality, daytime sleepiness, depressive and anxious symptoms, and quality of life. In our experience, sleep dysfunction in patients with HD is associated with increased anxiety and worse quality of life.

\section{8}

\section{How to Navigate the Huntington's Disease (HD) Big Data Maze?}

Muthukumaran Thangaramanujam, Jennifer Hoblyn

Bloomfield Hospital and Trinity College Dublin, Dublin, Ireland (all authors)

Background: It is currently difficult to predict the onset of symptoms, and disease progression as HD data is currently segregated like a maze. Aims and Objectives: This poster proposes an information model for HD Big Data, for linking all HD research data ranging from Basic Medical Research, Translational Medicine, and Clinical Outcomes.

Methods: An Information Model is a framework that can be used to categorize information resources. We propose The Hourglass Framework for HD Big Data.

Top of the hour glass: At the moment, the treatment a person with HD patient receives trickles down from existing research and clinical data which is still naescent. There appears to be disconnect between HD research and clinical practice in various parameters. It is proposed that a hyperlinked data set be created, covering from genetic data to clinical progression throughout the lifespan of the individual, and to have ethnicity specific population datasets. When the data acquisition is fine-tuned and streamlined as proposed, then this could trickle further on to the person with $\mathrm{HD}$, at the middle.
Centre: Person with HD.

Base of the Hourglass: The personalized targeted therapy outcomes are meticulously collected in an agreed standardized framework, and trickle down further to the data repository. The open non-relational database of outcomes could be open for data wrangling for clinicians to provide decision support thereby enabling targeted personalized medicine interventions. Eventually, the effective targeted outcomes are stored in an open relational database as a linked repository which would eventually form the HD Big Data Repository.

Conclusion: This Hourglass Digital Health Framework would thereby enable both clinicians to apply targeted personalized medicine treatment plans to individuals, and researchers to look beyond their speciality, thereby eventually guiding us to navigate the maze that is HD.

Lay Summary: Currently, HD research and clinical data lie segregated in an unstructured way. It's vital to have a global synergy to solve $\mathrm{HD}$, so we propose an information model or a "goggle" to navigate this HD Big Data maze.

89

Enroll-HD Platform Services

Enroll-HD is a global research platform with the infrastructure to support clinical trials and studies. Key elements include global study management and governance, an integrated EDC and study database and a user-friendly comprehensive webportal. This infrastructure supports the Enroll-HD study, a prospective, observational, longitudinal registry study of $\mathrm{HD}$, currently with $>18,000$ participants who have performed standardized clinical assessments and biosample collections from annual visits at over 163 clinical sites in 19 countries, and provides resources for clinical trial sponsors conducting interventional and observational studies in HD.

Resources and services of the platform that are available to the HD research community include easily accessible periodic clinical datasets and associated biosamples, assistance with site feasibility and selection, ICF development and translation, insurance, assessment scale licensing, IRB/ ethics submissions, site staff training and certification via the Enroll-HD training portal, database construction, and data monitoring.

Long term working relationships with the clinical sites allows for wellinformed site selection and efficient feasibility using Enroll-HD study data and the HD Clinical Trial Site Certification list (sites assessed against a set of standard minimum criteria for clinical trial participation), local knowledge of sites, recorded past performance of sites and access to eligible participants. The Enroll-HD platform's substantial registry of participants allows for powerful in-silico screening to support participant recruitment and the close working relationships with sites enables ongoing support for issue resolution and trial facilitation.

A team of subject matter experts (SMEs) with extensive experience in study start-up and management are available to assist in the implementation and operationalisation of clinical studies. The provision of platform services, including SME support, is managed by a team of Platform Resource and Service Managers (PRSM). This team also facilitates the interface between clinical study and trial project teams or researchers and the Enroll-HD Platform.

Lay Summary: The Enroll-HD platform infrastructure supports clinical trials and studies by providing resources and services to the HD research community, including clinical datasets and associated biosamples, assistance with site feasibility and selection, ICF development and translation, insurance, assessment scale licensing, training and certification, IRB/ ethics submissions, and participant recruitment.

90

\section{Huntington's Disease Clinical Trial Site Certification}

Enroll-HD Platform Team

CHDI Foundation, USA 
The Huntington's Disease (HD) Clinical Trial Site Certification Scheme aims to maximize the number of high-quality HD clinical trial sites, improve efficiency and expedite the site selection process for clinical trials. It provides an opportunity for HD clinical sites to register their interest and ability to participate in trials and increases the visibility of these sites to sponsors and clinical study teams. The scheme reduces the administrative burden during trial set-up by maintaining up to date centralized records about sites and providing these details to approved sponsors conducting HD clinical research. More than 100 sites around the globe have received certification, providing an invaluable resource to the HD clinical trial community.

Certification requires that sites meet a specific set of criteria designed to assess clinical trial readiness including clinical trial infrastructure and facilities, information on HD cohort and participant access arrangements, training, education and research experience. The scheme is open to Enroll-HD and non-Enroll-HD sites in Australia, Europe, Latin America, North America, New Zealand, Nordic regions and Russia, and is managed by the Enroll-HD Clinical Trial Committee (CTC). Sites apply for certification by completing a brief application form, completing the GSID-HD (HD Global Site Investigator Database) and submitting a standard set of documents and SOPs for review. For further information contact sitecertification@enroll-hd.org.

Lay Summary: The Huntington's Disease Clinical Trial Site Certification Scheme aims to maximize the number of high-quality HD clinical trial sites, improve efficiency and expedite the site selection process for clinical trials. It provides an opportunity for $\mathrm{HD}$ clinical sites to register their interest and ability to participate in trials and increases the visibility of these sites to sponsors conducting HD research.

\section{1}

\section{Registry Dataset (RDS)}

European Huntington's disease Network, on behalf of Registry investigators and participants

European Huntington's disease Network

Registry is an observational, prospective, longitudinal, multi-national, multi-centre cohort study of Huntington's disease (HD) initiated in 2004, which annually collected standardized clinical data and biospecimens on individuals affected by HD, HD family members and controls from 161 expert HD clinical sites across 17 European countries. Over 14,000 participants contributed clinical phenotypical data, with more than 10,000 participants additionally donating biosamples for use in large-scale genetic modifier and biomarker studies. This data is now available via the Registry dataset (RDS) (https://www.euro-hd.net/html/ projects/proposals/scipro), which is an invaluable resource for scientists and researchers conducting clinical and genetic modifier studies.

Some values in the RDS have been aggregated to further protect the identity of study participants. Access to disaggregated data and/or data not provided in the RDS can be applied for via a specified dataset request. The procedure for obtaining access to the RDS and renewable biosamples is straightforward and involves a review of the project by the Chairs of the European Huntington's Disease Network Scientific and Bioethics Advisory Committee (SBAC) and Executive Committee (EC). Requests for a specified dataset or nonrenewable biosamples will undergo full review by the SBAC and EC.

The Registry study was closed in 2017 and since 2013, many of the Registry sites and participants have been transitioning from Registry to Enroll-HD, the global observational study of HD (www.enroll-hd.org). The RDS has been prepared in a similar format to the datasets available from Enroll-HD (periodic datasets (PDS)), using the same recoded IDs, which simplifies the analysis for applicants requesting access to both Registry and Enroll-HD datasets. Some Registry data is also included in the Enroll-HD PDS for participants who have transitioned from Registry to the Enroll-HD study, however, there are a wealth of Registry data that are uniquely represented in the RDS.
Lay Summary: The Registry dataset (RDS) is a valuable resource for the HD scientific community, comprising annually collected standardized clinical data ( $>14,000$ individuals) and biospecimens $(>10,000$ individuals) on people affected by HD, HD family members and controls. The RDS is available to researchers via a simple application process: https:// www.euro-hd.net/html/projects/proposals/scipro.

\section{2}

Using Functional Status to Aid Interpretation of cUHDRS Scores in Patients with Huntington's Disease.

Dylan Trundell ${ }^{1}$, Giuseppe Palermo ${ }^{2}$, Jeffery D. Long ${ }^{3}$, Blair R. Leavitt ${ }^{4}$, Scott Schobel ${ }^{2}$, Sarah J. Tabrizi ${ }^{5}$

${ }^{1}$ Roche Products Ltd., Welwyn Garden City, UK; ${ }^{2}$ F. Hoffmann-La Roche Ltd., Basel, Switzerland; ${ }^{3}$ University of Iowa, Iowa City, USA; ${ }^{4}$ University of British Columbia, Vancouver, BC, Canada; ${ }^{5}$ Huntington's Disease Research Centre, UCL Institute of Neurology, London, UK

The composite Unified Huntington's Disease Rating Scale (cUHDRS) has been developed to assess disease progression in early-to-moderate manifest Huntington's disease (HD). It combines four assessments (Total Functional Capacity [TFC], Total Motor Score, Symbol Digit Modalities Test and Stroop Word Reading) into a single metric that provides more comprehensive sensitive outcome assessment of cognitive, functional and motor impairment in HD. To aid score interpretation, it is important to understand how cUHDRS score relates to typical functional status.

Data from Enroll-HD were evaluated. The cUHDRS score range was calculated for patients meeting the reference population for the Phase III RG6042 study, GENERATION HD1 (manifest HD, TFC 5-13, $\geq 20$ years; $N=3490$ ). Patients were divided into groups around each integer score (e.g. for $\mathrm{cUHDRS}=11: 10.5 \leq \mathrm{cUHDRS}$ score $<11.5$ ). For each grouping, the mean HD stage, Independence Scale (IS) score, mean Functional Assessment (FA) score and number of FA items that $\geq 50 \%$ of individuals can achieve were calculated.

In this population, cUHDRS score groupings ranged 2-18; however, six individuals were $\mathrm{cUHDRS}=2$, and so this grouping was excluded from subsequent analyses, leaving a range of 3-18 $(N=3484)$. For those patients in the $14-18$ cUHDRS score groupings, $\geq 50 \%$ were able to achieve all $25 \mathrm{FA}$ items and had a mean IS of 95. As cUHDRS scores declined, the number of FA items achievable and the level of independence declined. For patients in the lowest cUHDRS score group, cUHDRS $=3$ points, only 12 FA items were achievable by $\geq 50 \%$ and the mean IS $=65$. A visual aid was created using these results, to aid interpretation of cUHDRS scores, by showing how cUHDRS scores correspond to existing measures of functional ability.

Funding Information: This study was funded by F. Hoffmann-La Roche Ltd.

Lay Summary: Huntington's disease (HD) is a complex disease that causes problems with a person's ability to think, behave and move. Current measurements used in clinical trials do not comprehensively assess the impact of the disease. Scientists have developed an improved measurement of disease progression called the composite Unified Huntington's Disease Rating Scale. To aid interpretation of this measurement, a visual aid has been developed linking the outcomes of this measurement to other measures of functional ability in HD currently in use.

\section{3}

\section{Novel Exploratory Outcome Assessments in GENERATION HD1}

Dylan Trundell ${ }^{1}$, Scott A. Schobel ${ }^{2}$, Raymund Roos ${ }^{3}$, Karen Anderson ${ }^{4}$, Alpa Thobhani ${ }^{1}$, Gregory Hooper ${ }^{1}$, Mai-Lise Nguyen ${ }^{2}$, Sarah J. Tabrizi ${ }^{5}$ ${ }^{1}$ Roche Products Ltd., Welwyn Garden City, UK; ${ }^{2}$ F. Hoffmann-La Roche Ltd., Basel, Switzerland; ${ }^{3}$ Leiden University Medical Centre, Leiden, the Netherlands; ${ }^{4}$ Georgetown University Hospital, 
Washington, DC, USA; ${ }^{5}$ Huntington's Disease Research Centre, UCL Institute of Neurology, London, UK

With multidimensional diseases such as Huntington's disease (HD), there is a need to assess a broad range of symptoms and impacts. Although novel patient-reported outcome measures (e.g. HD-PRO-TRIAD, HDHealth Index, FuRST 2.0) have been developed, there is still need for a clinician-reported outcome (ClinRO) measure of daily function that can assess change in a more granular way than existing ClinROs.

Gaps were identified while evaluating the holistic measurement strategy for the first pivotal Phase III study, GENERATION HD1, against qualitative research, indicating the core symptoms and impacts from the perspectives of patients and their families. It was determined that in addition to a novel ClinRO assessing daily functional ability, brief measures of patient-reported speech difficulty and companion-reported irritability were also required to balance patient burden with the desire for comprehensive measurement. Qualitative research was reviewed and, in collaboration with HD clinical experts, an iterative process was used to develop items and response options to assess clinician-reported daily functional ability.

Additionally, a single-item patient-reported assessment of speech difficulty and three draft companion-reported items to assess observed irritability and angry outbursts were developed. The draft companion-reported measure was shared with the international HD-COPE board of patients and care companions via an interactive online platform. Feedback was used to revise the content.

Three novel scales were developed and are being used for the first time in the GENERATION HD1 study as exploratory measures: the HD-Daily Activities Scale, a 25-item measure of daily activities; the HDCompanion-reported Irritability and Angry Outbursts Scale, a 2-3 item measure of irritability and angry outbursts; and the HD-Speaking Difficulty Item, a single-item measure of speech difficulty.

Future assessment of their measurement properties (e.g. validity and reliability) is required to support their use in longitudinal research.

Funding Information: This study was funded by F. Hoffmann-La Roche Ltd.

Lay Summary:Scientists have developed three new measurement tools to better understand how Huntington's disease (HD) impacts a person's speech, their ability to complete day-to-day tasks and how often they experience irritability or angry outbursts. The measures were developed with HD experts and feedback from the global patient/companion advisory board HD-COPE. These measures will be used for the first time in the Roche RG6042 Phase III GENERATION HD1 clinical trial.

94

Huntington's Disease: Disclosure and Future Decision-Making in Romantic Relationships

Marian Tsang, Moyra Smith, Leslie Thompson, Katherine Hall

University of California Irvine, Irvine, CA, USA (all authors)

Huntington's Disease (HD) is an inherited neurodegenerative condition in which an affected individual gradually loses control over their behavior, cognition, and movement. Typically, onset of symptoms is in the 30 s or $40 \mathrm{~s}$, although there are some cases that fall outside of this age range. This means most individuals often achieve early to mid-life milestones, including romantic relationships, marriage, and reproduction, before the onset of symptoms. An individual with HD has a $50 \%$ chance of passing down the condition to each child. If an individual decides to disclose to a potential romantic partner their HD history, communicating the symptoms and inheritance aspects of the condition may help the couple make informed decisions regarding their possible future life together. How and when individuals with, or at risk for, HD approach dating, relationships, disclosure, and life decisions with a potential romantic partner is a topic that has not been well documented in the literature. This information can give healthcare providers deeper understanding into this important aspect of life for their patients. This study surveyed individuals both at risk for and diagnosed with HD, as well as their romantic life partners regarding issues surrounding discussion of the diagnosis of HD. The survey included both multiple choice and free response questions on the subject of disclosure and its impact on reproductive decisions including family planning and in vitro fertilization with preimplantation genetic testing (PGT$\mathrm{M})$. The information collected in this study provides insight into disclosure and factors considered by those affected and at risk for HD and their romantic partners as they consider their future together.

Lay Summary: This study explored the area of disclosure and family planning decisions from the perspectives of the individual who was either at risk for or diagnosed with Huntington's disease and the romantic partners of these individuals. The goal of this study is to give healthcare providers important insight into this aspect of life for their patients.

\section{5}

Speech in Prodromal and Symptomatic Huntington's Disease as a Model of Measuring Onset and Progression in Dominantly Inherited Neurodegenerative Diseases

Jess C.S. Chan ${ }^{1}$, Julie C. Stout ${ }^{2}$, Adam P. Vogel ${ }^{1,3,4}$ *

${ }^{1}$ Centre for Neuroscience of Speech, University of Melbourne, Victoria, Australia; ${ }^{2}$ Institute of Cognitive and Clinical Neuroscience, School of Psychological Sciences, Monash University, Victoria, Australia; ${ }^{3}$ Department of Neurodegeneration, Hertie Institute for Clinical Brain Research, University of Tübingen, Germany; ${ }^{4}$ Redenlab, Australia

Subtle motor and cognitive changes can manifest prior to diagnosis in many progressive neurological disorders. Most people with Huntington's disease (HD) develop dysarthria. Yet, how speech evolves, how the brain regulates speech in $\mathrm{HD}$, and the clinical implications of these changes remain unclear. We reviewed existing literature on speech production in premanifest (PreHD) to symptomatic HD. A search of databases yielded 12 eligible studies. Nine of the twelve studies were included in the metaanalysis. Data suggest that speech in PreHD is characterized by impaired speech agility, phonatory function and speech rate. Over the disease course, speech gradually deteriorates across all subsystems of speech. Our findings indicate subtle changes in speech appear prior to a clinical diagnosis of HD. However, distinct patterns of decline and the magnitude of these deficits require further investigation. Our current understanding of speech impairment in HD relies on small cohort studies. Natural history studies documenting changes from premanifest to end stage may facilitate the development of speech as a marker of disease onset and treatment response.

Lay Summary: •Subtle but apparently genuine changes in speech occur in HD before clinical diagnosis. $\cdot$ Speech changes in prodromal individuals are largely characterized by timing deficits. $\cdot$ Limited longitudinal data restricts capacity to predict HD evolution using speech.

\section{6}

Motor Speech Phenotype of Huntington's Disease: A Clinical Biomarker in Premanifest and Early Phases

Jess C.S. Chan ${ }^{1}$, Julie C. Stout ${ }^{2}$, Adam P. Vogel ${ }^{1,3,4} *$

${ }^{1}$ Centre for Neuroscience of Speech, University of Melbourne, Victoria, Australia; ${ }^{2}$ Institute of Cognitive and Clinical Neuroscience, School of Psychological Sciences, Monash University, Victoria, Australia; ${ }^{3}$ Department of Neurodegeneration, Hertie Institute for Clinical Brain Research, University of Tübingen, Germany; ${ }^{4}$ Redenlab, Australia

Background: Speech disturbances have been described in premanifest Huntington's Disease (HD). Yet a recent systematic review and metaanalysis highlighted disagreement in the literature regarding the nature 
and size of these deficits. How speech changes over time is relatively unknown outside of single time point cohort studies.

Objective: Drawing on measures known to be sensitive to pathology, we explored the utility of objective analysis of diadochokinesis (DDK) in describing four distinct HD groups and the stability of those measures over time in the premanifest phase.

Methods: Sensitivity of speech metrics was determined by comparing production in 55 people carrying the expanded HD gene divided into four groups (14 presymptomatic (PresymHD); 18 prodromal (ProHD); 14 early (EarlyHD); and 9 middle (MidHD) stages of HD). Participants' speech data were compared to four groups of age- and sex-matched control subjects $(n=55)$. Participants repeated syllable sequences, sustained vowel, read a passage and produced a monolog. Stability was investigated in a subset of 13 participants from the two premanifest groups by measuring speech over a 6-months period (7 days, 1 month, 3 months and 6 months post baseline). All speech data were analyzed objectively using acoustic methods.

Results: Speech performance did not differ between control and PresymHD (further from disease onset) groups. There were significant differences on measures of syllabic duration and syllable repetition rate between participants with prodromal HD (within 15 years to disease onset) and their matched controls. People in the ProHD group also presented with slower rate of speech in reading tasks with greater pauses and silences compared to controls. Results from stability data revealed differences in speech function between PresymHD and ProHD groups over the 6-months period, and there were no significant differences across the five time points within each group. As the disease progresses, speech agility and timing appear to gradually deteriorate.

Conclusion: Articulatory rate and agility are significantly reduced in individuals closer to the onset of HD. The speech decline observed may be due to task novelty in syllable repetition task, as well as cognitive (e.g. attention) and oromotor changes in the premanifest period. Our data suggest that these features are relatively stable over a six-month period.

Lay Summary: Subtle motor speech differences appear to be detectable more than a decade from disease onset using objective analysis of speech. We explored the stability of speech measures over a six-month period in a subset of participants in the premanifest phase of HD. Data show relatively stable production over brief and intermediate inter-recording intervals.

\section{7}

GEORGE $\AA^{\circledR}$ - The First Smartphone Application for Huntington Disease - Pilot Study

Emma M. Waddell ${ }^{1}$, Karthik Dinesh ${ }^{2}$, Kelsey L. Spear ${ }^{3}$, Christopher G. Tarolli ${ }^{1}$, Molly J. Elson ${ }^{4}$, Michael J. Curtis ${ }^{5}$, David J. Mitten ${ }^{5}$, Gaurav Sharma $^{2}$, E. Ray Dorsey ${ }^{1}$, Jamie L. Adams ${ }^{1}$

${ }^{1}$ University of Rochester Center for Health + Technology, Rochester, NY, USA; ${ }^{2}$ University of Rochester Department of Electrical and Computer Engineering, Rochester, NY, USA; ${ }^{3}$ Chicago Medical School at Rosalind Franklin University, North Chicago, IL, USA; ${ }^{4}$ Emory University School of Medicine, Atlanta, GA, USA; ${ }^{5}$ University of Rochester Health Lab, Rochester, NY, USA

Objective: Assess the feasibility of using an Android smartphone application, GEORGE®, to quantitatively measure motor features of Huntington disease.

Background: Current measures of Huntington disease are insensitive, subjective, and restricted to in-person visits. As technology becomes increasingly inexpensive, ubiquitous, and advanced, smartphones present a unique opportunity to objectively measure disease. Previous studies in Parkinson disease have demonstrated that smartphones effectively measure motor symptoms and correlate well with clinician assessments.

We developed the first smartphone application for HD, GEORGE®, at the University of Rochester. This technology may differentiate individuals with and without $\mathrm{HD}$, quantify motor symptoms, and detect response to drug treatment. GEORGE® uses the smartphone's sensors to assess chorea and balance, the screen to assess tapping speed, and the microphone to assess voice.

Methods: This is an observational study of 25 participants: 10 with manifest HD, 5 with prodromal HD, and 10 healthy controls. Participants undergo two in-clinic assessments and complete the application's tasks three times daily for one month. Primary outcome measures include feasibility and quantitative motor data.

Results: To date, 23 subjects have been enrolled, and one withdrew before completing any remote tasks. On average, participants use the application 2.3 times daily, with no significant difference in frequency based on disease status. We have observed a significant difference in speed of finger tapping, gait regularity, and chorea score derived from the application between individuals with and without manifest HD. Individuals with prodromal $\mathrm{HD}$, the youngest cohort, tapped at the fastest rate and had the lowest average chorea score.

Conclusions: Our data suggest use of a smartphone application by individuals with $\mathrm{HD}$ is feasible. GEORGE® successfully differentiates individuals with and without manifest HD and measures disease features. Refinement of the application's interface and activities will improve usability and sensitivity of data and, ideally, make it useful for clinical trials.

Lay Summary: Developed at the University of Rochester, GEORGE® is the first smartphone application for HD. This novel technology has the potential to distinguish between individuals with and without HD, quantify motor symptoms, and measure response to drug treatment. By enabling faster and more frequent data collection, GEORGE® could expedite the development of novel therapeutics for HD.

\section{8}

Evaluation of Effector Domains Fused to dCas9 for Allele-specific Silencing in Huntington's Disease Patient-derived Cells

Jennifer Waldo ${ }^{1,2}$, Peter Deng ${ }^{1,2,3}$, Julian Halmai ${ }^{1,2}$, Jasmine Carter ${ }^{1,2}$, Casiana Gonzales $^{1,2,4}$, Samantha Del Campo ${ }^{1,2}$, David Cameron ${ }^{1,2}$, Jan Nolta $^{1}$, David Segal ${ }^{3}$, Kyle Fink ${ }^{1,2}$

${ }^{1}$ Stem Cell Program and Institute for Regenerative Cures, University of California Davis Health Systems, Sacramento, CA, USA; ${ }^{2}$ Department of Neurology, University of California Davis Health Systems, Sacramento, CA, USA; ${ }^{3}$ Genome Center, MIND Institute, and Biochemistry and Molecular Medicine, University of California, Davis, CA, USA; ${ }^{4}$ Department of Biological Sciences, California State University, Humboldt, CA, USA

Huntington's disease (HD) is caused by a trinucleotide expansion in exon 1 of the Huntingtin gene, which leads to neuronal dysfunction and eventually cell death. The prospect of allele-specific reduction is important, as it is not known what the consequences of total knockdown of the Huntingtin protein could be in the long term. We aimed to use single nucleotide polymorphisms (SNPs) found in the patient population to reduce the expression of only the mutant HTT with a modified CRISPR system in HD patient-derived cells. We first characterized disease-associated single nucleotide polymorphisms (SNPs) in patient-derived primary cells that could be used to guide our modified CRISPR system to the mutant allele. We confirmed heterozygous SNPs near regulatory regions of the Huntingtin promoter in HD patient cells which allowed for the design of allele-specific guide RNAs containing the SNP proximal to the PAM site, which is needed for the Cas9 protein to recognize and bind to DNA.

We then cloned a new Cas9 variant, $\operatorname{dxCas}(3.7)$, tethered to repressive effector domains into a novel vector via Gibson cloning (dxiCas9). $\mathrm{dxCas}(3.7)$ has been shown to have a broader variety of PAM sites and higher binding specificity, which is necessary to target the mutant-allele associated SNPs in the HTT promoter. The various dxiCas9 and gRNA plasmids were introduced to an HD patient-derived fibroblast line as an initial proof-of-concept study. After delivery of our gRNAs and different dxiCas proteins, we performed qRT-PCRs to assess knockdown of total HTT transcript levels as a screen for gRNA selection to continue with. We 
assessed at knockdown at 4 different loci in the HTT gene using various gRNAs and effector domains, and saw downregulation using several of our gRNAs that were designed to be allele specific.

Lay Summary: We are currently working towards therapeutics using gene editing technology to turn off the mutant gene that causes HD. Targeting mutant huntingtin at the DNA level could allow for a more substantial effect and prolonged effect.

\section{9}

\section{Music Therapy to Address Issues in Huntington's Disease}

\section{Becky Wellman}

Cleveland Clinic Lou Ruvo Center for Brain Health, Las Vegas, Nevada, USA

Music therapy is a clinical and evidenced-based intervention which can accomplish individual goals within a therapeutic relationship led by a credentialed provider. Music can be an effective tool in addressing the physical, communication, behavioral, and emotional symptoms of Huntington's disease (HD). Using the innate elements of music such as rhythm, melody, beat, etc., music therapists can aid in regulating gait, increasing diction and annunciation of speech, control fine motor skills, and increase coping skills for and ease emotional concerns associated with HD. Case reports of two patients, information regarding a caregiver/patient support group, and ways to utilize music therapy in other settings will be shared.

Case 1: Specific goal areas of breath endurance, articulation, voice inflection, volume, and oral motor coordination were addressed utilizing singing, vocal exercises, and wind instrument play. Goal areas of safe ambulation, motor coordination, and defined fine motor movement were addressed through instrument play and rhythmic auditory stimulation.

Case 2: Specific goal areas of maintaining respiratory support, fine motor function, and cognitive skills were addressed utilizing instrument play and singing. Goal areas of reduced anxiety were addressed utilizing progressive relaxation and Guided Imagery and Music as well as a therapeutic listening program.

Support Group: The intent of adding music therapy was to allow opportunities for the patients to receive therapeutic support within a positive, success oriented group process while the caregivers were able to meet with a psychologist to discuss their own needs and concerns. Goals included increased communication, emotional support, opportunities for self-expression, cognitive stimulation, and social engagement. These goals were addressed through group singing, song writing, music discussion, cognitive music games, and instrument play.

Lay Summary: Music therapy can provide non-pharmacological support for motor, communication, behavioral, and emotional concerns presented with Huntington's disease. We will show examples of how music therapy has directly impacted patients with HD as well as how other facilities/ families can add music therapy to their plan of care.

100

\section{Prevalence of Huntington's Disease in the US}

George Yohrling ${ }^{1}$, Karina Raimundo ${ }^{2}$, Valerie Crowell ${ }^{3}$, Debra Lovecky $^{1}$, Louise Vetter ${ }^{1}$, Lauren Seeberger ${ }^{4}$

${ }^{1}$ Huntington's Disease Society of America (HDSA), New York, NY, USA; ${ }^{2}$ Genentech Inc., South San Francisco, CA, USA; ${ }^{3}$ F. HoffmannLa Roche, Ltd., Basel, Switzerland; ${ }^{4}$ Department of Neurology, University of Colorado, Denver, CO, USA

Huntington's disease (HD) is a genetic, neurodegenerative and ultimately fatal disease. Sources frequently state that 30,000 individuals living in the US have HD, but the methodology used to derive this prevalence estimate is unclear. We aimed to review the existing literature on the prevalence of HD in the US and Canada and to estimate the number of people in the US currently affected by this disease.
We reviewed the literature on HD prevalence in Canada and US post the advent of genetic testing (1993-2019) and calculated a range of persons affected in the US by age-adjusting these prevalence estimates to the US population using 2018 US Census data.

Two studies were identified (Fisher, et al. 2014 and Bruzelius, et al. 2019). Fisher performed a population-based observational study using multiple ascertainment sources and evaluating clinical and genetic data of people with HD in British Columbia (BC), Canada, which yielded an estimated prevalence of 13.7/100,000 (US age-adjusted: 12.7/100,000). Bruzelius conducted a retrospective database analysis of insurance claims of one insurance company and estimated an age-adjusted HD diagnostic frequency of 6.52/100,000. Applying these age-adjusted prevalence rates to the 2018 US census population, we estimated the true HD prevalence in the US to be 41,467 , while the number of persons currently diagnosed is at least 21,331 .

Adjusting for differences in the BC, and US populations, our assessment indicates that as many as 40,000 US persons may currently have manifest HD. It is probable that many of these patients go undiagnosed. The lower figure ( 21,000 patients) may reflect the number of patients currently diagnosed but does not account for the HD prevalence in uninsured individuals. With the advent of disease-modifying treatments, HD disease awareness and diagnosis rates may increase, enabling improved detection of patients. Funding Information: Study sponsored by Genentech Inc. and F. Hoffmann-La Roche Ltd.

Lay Summary: This study proposes an update to the estimated number of individuals living with Huntington's disease (HD) in the US. It is often reported that there are 30,000 individuals living with HD in the US, but the methods used to estimate this number are unclear. Based on information from two population studies, a claims database of a medical insurance company and a study of patient charts, accounting for US census data; the authors of this study found that as many as 40,000 people may have manifest HD in the US.

101

Ancillary Service Utilization and Impact in Huntington's Disease

Jacob Yomtoob, Chen Yeh, Danny Bega

Northwestern University Feinberg School of Medicine, Chicago, IL, USA (all authors)

Background: Prior Huntington's disease (HD) studies suggest ancillary services improve motor symptoms, cognition, mood, and quality of life but frequency of use and clinical characteristics are unclear.

Objective: Describe ancillary service utilization in a cohort of individuals with HD and determine which participant characteristics are associated with ancillary service utilization.

Methods: Retrospective cross-sectional analysis of Enroll-HD database. Participants were grouped by therapy: physical and/or occupational (PT/ OT), psychotherapy and/or counseling (PC), speech and/or swallowing (ST). We performed bivariate comparisons analysis of demographic and disease characteristics between those with/without each therapy and to analyze one-year mean change in assessment scores.

Results: Of 4751 participants, 1537 (32.35\%) utilized therapies $(11.82 \%$ PT/OT, $5.33 \%$ PC, $3.01 \%$ ST, $1.98 \%$ all three, $10.21 \%$ two therapies). PT/OT participants had worse motor and functional scores: mean UHDRS motor score ( 41.17 vs. $38.05, p=0.002)$, median total functional capacity score (TFC) $(8.00$ vs. $9.00, p<.001)$. PC participants had worse mood but better cognitive and functional scores: median depression score (7.00 vs. $2.00, \mathrm{p}<.001)$, median MMSE (28.00 vs. $26.00, \mathrm{p}<.001)$, median TFC $(10.00$ vs. $8.00, p<.001)$. ST participants had more dysarthria, and worse cognitive and functional scores: dysarthria $(32.2 \%$ vs $20.1 \%$ p <.001), mean correct Symbol Digit Modality Test (16.79 vs. $23.27, \mathrm{p}<.001)$, median TFC $(6.00$ vs. $9.00, \mathrm{p}<.001)$. Over one year, PC participants' depression scores improved compared to untreated $(-1.24$ vs. $-0.11, p=0.040)$. ST participants' depression scores worsened 
(1.14 vs. $-0.23, p=0.044)$. Mean change in TFC was not significant for any therapies.

Conclusions: Only $32 \%$ of Enroll-HD site participants received ancillary services. Use correlated with expected clinical characteristics, though impact of use remains unclear.

Lay Summary: Prior Huntington's disease studies have suggested that ancillary services (including physical therapy, occupational therapy, psychotherapy, counseling, speech therapy, and swallowing therapy) improve motor symptoms, cognition, mood, and quality of life but frequency of use and clinical characteristics correlating with use are unclear. We aimed to describe use of these services in the large worldwide Enroll-HD cohort and examine how participant characteristics were associated with different service use. Only $32 \%$ of study participants had received these services. Our study will better inform referral practices to make these vital resources available to more people with HD.

\section{2}

Using Propensity Scores to Balance Covariates in Exercise Trials in People with Huntington's Disease (HD)

Gregory Youdan $\mathrm{Jr}^{1}$, Xueyao $\mathrm{Li}^{1}$, Beth Ann Griffin ${ }^{2}$, Rebecca Playle ${ }^{3}$, Monica Busse ${ }^{3}$, Lori Quinn ${ }^{1,3}$

${ }^{1}$ Teachers College, Columbia University, New York, NY, USA; ${ }^{2}$ Rand Corporation, Santa Monica, CA, USA; ${ }^{3}$ Centre for Trials Research, Cardiff University, Cardiff, UK

Background: Achieving sufficiently powered trials of exercise and other life-style interventions in Huntington's Disease (HD) are difficult not least due to the small, geographically dispersed population. Observational studies together with propensity score models (PSM) can be used to estimate causal effects of treatment without requiring randomization by accounting for the key confounding covariates that predict both receiving treatment and the outcome of interest.

Methods: Data from five feasibility exercise RCTs, not powered to detect difference (i.e., Move to Exercise, COMMET, TRAIN, EXERT, and ENGAGE) were pooled together using an individual patient data (IPD) meta-analysis. To account for missingness, 30 multiple imputations were performed on baseline covariates using MICE in R and results were pooled using Rubin's rules. PSM was used to match individuals in exercise groups and control groups. Covariate balance was assessed using a criterion of $<0.1$ mean difference and variance ratios to signify good balance. The PSM was created using a logistic regression and an optimal matching approach (OptMatch in R) was used to compare groups.

Results: There was a 1.44 (95\% CIs [-.65, 3.53]) simple mean difference in modified motor score (mMS) in favor of the control group. After balancing covariates using PSM, this difference changed to be in favor of the exercise group with an average treatment effect of treated (ATT) of -0.88 (95\% CIs $[-0.86,-0.91])$.

Conclusions: Although each study was independently randomized (different minimization variables), when combined (using the IPD) the groups were unbalanced on baseline covariates. Correcting the imbalance by using PSM reversed the signal to provide weak indication that exercise might improve motor function. PSM may provide a better way to estimate causal effects of exercise when large scale randomized controlled trials (RCTs) are not feasible, and researchers must rely on using large observational cohort studies such as ENROLL-HD.

Lay Summary: Propensity Score Models (PSM) can be used to make causal inference in observational studies where randomization may not be feasible. Data from five feasibility exercise RCTs were pooled together and analyzed with PSM showing a weak indication that exercise may improve motor function in people with HD, after covariates were balanced between exercise exposure and control group. PSM should be considered as a viable approach by HD researchers, especially in lieu of large observational cohort studies such as ENROLL-HD.
103

A Systematic Review of the Clinical Evidence for Complementary and Alternative Medicine in Huntington's Disease

Margaret Yu, Danny Bega

Northwestern University Feinberg School of Medicine, Chicago, IL, USA (all authors)

Background: There is a lack of published guidelines related to the use of Complementary and Alternative Medicine (CAM) for Huntington's disease (HD). We conducted a systematic review of the literature to summarize the available evidence for various mind-body practices and nutraceuticals.

Methods: PubMed and Cochrane Library electronic databases were searched independently from inception to February 2019 by two independent raters. Studies were classified for the level of evidence (class I, II, III, or IV) according to the American Academy of Neurology (AAN) classification scale.

Results: Randomized controlled trials in HD were reviewed for mindbody interventions (dance therapy, music therapy, and exercise), alternative systems (traditional Chinese medicine), and nutraceuticals/diet (aminooxyacetic acid, coenzyme q10, creatine, cannabinoids, alpha-tocopherol, eicosapentaenoic acid, idebenone, levocarnitine, triheptanoin). Few studies met AAN class I or II level of evidence for benefits, and these are highlighted.

Discussion: There is a relative paucity of clinical trials examining CAM modalities in HD when compared to other neurodegenerative disorders. Presently there is no evidence supporting disease modification or symptom improvement with any specific dietary or nutraceutical supplement for HD. Supervised exercise and contemporary dance are safe for people with HD but more robust studies are warranted to guide specific recommendations for these and other mind-body interventions.

Lay Summary: Huntington's Disease (HD) does not have curative medications, only symptomatic treatment. Complementary alternative medicine has been found to be helpful in other neurodegenerative disorders like Parkinson's disease and Alzheimer's disease, but has not been investigated for HD. This review bridges this current gap in knowledge.

\section{4}

\section{Validity of Irritability in Huntington's Disease: A Scoping Review}

Jane Simpson ${ }^{1}$, Maria Dale ${ }^{2}$, Rachael Theed ${ }^{1}$, Sarah C Gunn ${ }^{3}$, Nicolò Zarotti ${ }^{1}$, Fiona Eccles ${ }^{1}$.

${ }^{1}$ Division of Health Research, Lancaster University, Lancaster, LA 1 4YT, UK; ${ }^{2}$ Adult Mental Health Psychology, Leicestershire Partnership NHS Trust, Leicester, LE4 8PQ, UK; ${ }^{3}$ Department of Neuroscience, Psychology and Behaviour, University of Leicester, Leicester, LE1 7RH, UK.

Purpose: To scope the literature concerning irritability in Huntington's disease to determine whether or not irritability is a valid and meaningful construct within this population.

Method: A scoping literature review was conducted based on findings from a search of five databases (Academic Search Ultimate, PsycINFO, CINAHL, Scopus and Web of Science) in November 2018. From an initial return of 453 papers, 40 were found suitable for review.

Results: Review of the 40 studies highlighted several aspects of irritability in people with HD which influence its validity as an independent construct in context of the disease. While various measures are used to assess irritability, a gold standard has yet to be identified and consequently irritability is assessed inconsistently across the literature. In addition, the results suggest that irritability may not reflect pathological disease processes in HD, but rather comprises a multidimensional construct which appears to be 
strongly associated with other psychological difficulties such as depression and anxiety.

Conclusions: The current concept of irritability in people with HD continues to lack a general consensus in the clinical literature, in terms of both operationalisation and assessment. Consequently, further research is warranted in order to determine the extent to which irritability is a valid construct within the context of HD, including its associated behavioral, cognitive and affective dimensions.

Lay Summary: Based on the review of the literature which is currently available, no satisfactory definition of irritability within the context of HD appears to exist. Indeed, considering the correlates of irritability, including depression, apathy and anxiety, these may currently provide more meaningful information about a person's experience. Additionally, current treatment options appear designed to treat these associated psychological difficulties rather than specifically targeting irritability. However, the present investigation does not exclude or cast doubt on the reality of what is described as irritability for many individuals affected by HD and their clinicians, but suggests that if effective help is to be provided, it needs to be much clearer on all aspects of this often extremely distressing experience.

\section{5}

Systemic Administration of Exosomal siRNA Inhibited Huntingtin Expression in Transgenic Mice of Huntington's Disease

Tengteng $\mathrm{Wu}^{1}$, Mengchao $\mathrm{Yu}^{2}$, Li Zhang ${ }^{2}$, Xi Chen ${ }^{2}$, Zhong Pei ${ }^{1}$ ${ }^{1}$ Department of Neurology, the First Affiliated Hospital, Sun Yat-Sen University; ${ }^{2}$ State Key Laboratory of Pharmaceutical Biotechnology, NJU Advanced Institute of Life Sciences, Jiangsu Engineering Research Center for MicroRNA Biology and Biotechnology, School of Life Sciences, Nanjing University

Cell-derived exosomes have been demonstrated to be efficient carriers of small RNAs into brain tissue. However, the therapeutic effect of exosome-mediated siRNA has not been tested in Huntington's disease. In the present study we aimed to explore the therapeutic potential of exosome-mediated siRNA delivery in HD mice models.

We employed modified exosomes expressing the neuron-specific rabies viral glycoprotein (RVG) peptide to deliver siRNA into brain tissue. To determine the ability of modified exosomes selectively entering brain tissue, eGFP-siRNA loaded exosome was administrated to eGFP transgenic mice. Five siRNA targeting human huntingtin exon 1 (HuHtt) transcript were designed and then screened in $\mathrm{SH}-$ SY5Y cells. To evaluate the in vivo efficiency, HuHtt-siRNA RVGexosomes were intravenously injected to BACHD mice at $10 \mathrm{mg} / \mathrm{kg}$ every two days for 2 weeks. The most effective HuHtt-siRNA RVGexosomes were then intravenously injected to N171-82Q and YAC 128 transgenic mice at $10 \mathrm{mg} / \mathrm{kg}$ twice per week for 2 to 6 weeks. Performance of rotarod test and balance beam test was determined post treatment.

We found that RVG modified exosomes efficiently and specifically delivered siRNA into the mouse brain. In addition, siRNA-loaded RVG exosomes reduced $\mathrm{HuHtt}$ mRNA $(55 \% \pm 13 \%)$ and protein $(51 \% \pm$ $14 \%$ ) in N171-82Q mice as well as improved rotarod performance. Similarly, exosomal siRNA significantly inhibited huntingtin expression and improved motor function in YAC128 mice.

The present study demonstrates that systemic exosomal siRNA efficiently lowers HTT and improves disease-phenotypes. Thus, systemic exosomal siRNA has a great potential for affordable, non-invasive solution for HTT lowering therapy.

Lay Summary: Systemic exosomal siRNA efficiently lowers HTT and improves disease-phenotypes. Thus, systemic exosomal siRNA has a great potential for affordable, non-invasive solution for HTT lowering therapy.
106

Coping Humor and Cognitive Reframing by Huntington's Disease Caregivers

Robin Zingales-Browne, Joy Elwell, Regina Barreca, Christopher Napolitano

University of Connecticut School of Nursing (all authors)

There is nothing humorous about Huntington's Disease (HD). It is a slow, progressive neurodegenerative disease that affects cognition, mood and coordination. HD is an autosomal dominant disease meaning offspring have a 50\% chance of inheriting it from one affected parent (Pickett, Altamaier, and Paulsen, 2007). There is no cure and it is eventually fatal. Treatment focuses on management of symptoms and palliative care. Yet even with this daunting disease, patients and families are initiating laughter in the clinic, exam rooms and in support groups. The humor is not in telling jokes, making fun or being cynical, sarcastic or degrading. The humor serves as a form of coping, a brief distraction from the disease and in a sense, hope for the future. A pilot study using humor in cognitive reframing was conducted to enhance caregivers' coping and self-efficacy. Caregivers attended two classes and completed a homework assignment. A thematic analysis revealed 5 shared concepts: control, emotion, humor, reframing and laughter. The results validate the need for future research and programs using coping humor and reframing with other healthcare populations.

Lay Summary: This is a creative program to improve self-efficacy and coping for Huntington's Disease caregivers. Cognitive reframing using humor can turn negative perceptions into more optimistic views. Caregivers who believe they can change their perceptions (self-efficacy) will have improved well-being and confidence to face the challenges of HD.

107

Characterization of Depressive Symptoms in Individuals with Reduced Penetrance Huntington's Disease Allele Expansions using Enroll-HD

Christina Dailey, Tara Newcomb, Emily Spoth, Meghan Zorn University of Utah, Salt Lake City, UT, United States (all authors)

Huntington's Disease is a rare neurodegenerative condition characterized by a triad of motor, cognitive, and psychiatric symptoms caused by a trinucleotide repeat expansion in the HTT gene. Individuals with 40 or more CAG repeats experience classic symptoms of the disease. Reduced penetrance alleles in Huntington's Disease occur when there are 36-39 CAG repeats in the HTT gene and pose a challenge to clinicians due to their variable nature. Recent studies have suggested that reduced penetrance alleles are more frequent in the general population than previously anticipated.

Some individuals with reduced penetrance alleles will have motor signs of the disease and others will not. However, the psychological symptoms affecting individuals with reduced penetrance alleles have not been investigated thoroughly. Depression is one of the most common psychiatric manifestations in Huntington's Disease and can occur before the onset of motor symptoms; however, the risk for depressive symptoms has not been examined in patients with reduced penetrance alleles. More comprehensive information surrounding the psychological symptoms affecting individuals with reduced penetrance alleles could aid health professionals to provide the best anticipatory guidance and aid in advocating for medical services.

It is hypothesized that there will be distinct psychological phenotypic differences between those with reduced penetrance Huntington's allele expansions, full penetrance Huntington's allele expansions, and normal 
allele lengths that could be used to guide medical care. The Enroll-HD Periodic Dataset 4, comprised of multiple psychological assessments from over 15,000 participants, will be used in conjunction with statistical software to analyze psychological manifestations of Huntington's Disease in reduced penetrance allele carriers compared to full penetrance allele carriers and those with normal allele lengths.

Lay Summary: Individuals with Huntington's Disease reduced penetrance alleles (36-39 CAG repeats) represent a unique patient population. It is unknown whether these individuals are at risk for the same psychological symptoms as those with full penetrance Huntington's Disease alleles. This study will use the Enroll-HD dataset to examine the psychological differences in the reduced penetrance allele patient population to provide better anticipatory guidance to healthcare providers. 